\title{
THE GEOCHRONOLOGICAL FRAMEWORK OF THE IRUMIDE BELT: A PROLONGED CRUSTAL HISTORY ALONG THE MARGIN OF THE BANGWEULU CRATON
}

\author{
B. De WAELE*, I.C.W. FITZSIMONS*, M.T.D. WINGATE**, F. TEMBO***, \\ B. MAPANI ${ }^{\S}$, and E. A. BELOUSOVA ${ }^{\S \S}$
}

\begin{abstract}
Ion microprobe U-Th-Pb analyses of zircon from 40 granitoid rocks collected from the late Mesoproterozoic Irumide Belt in Central Southern Africa, along the southern margin of the Archean to Paleoproterozoic Bangweulu Block, provide a comprehensive set of age data for this complex orogen. The data indicate that the Irumide Belt is constructed on a basement of principally Paleoproterozoic (ca. 2.05-1.93 Ga) age with a subordinate Neoarchean (ca. $2.73 \mathrm{Ga}$ ) component, which is overlain by a platformal quartzite-pelite succession known as the Muva Supergroup. Previously published U-Pb detrital zircon data for the Paleoproterozoic Muva Supergroup, which show age populations that match all of the pre-1.9 Ga basement components identified within the Irumide Belt, suggest that the pre-Muva basement was assembled as a coherent block by $\sim 1.8 \mathrm{Ga}$, which we refer to as the Bangweulu Craton. The southern margin of the Bangweulu Craton was then intruded by a previously unrecognized suite of biotite-bearing granitoid rocks between 1.66 and $\mathbf{1 . 5 5}$ $\mathrm{Ga}$, not recorded elsewhere in the region, and was later the site of emplacement of voluminous granitoid magmatism during the Irumide Orogeny at between 1.05 and $1.00 \mathrm{Ga}$. Hf isotopic data from zircon in these suites indicate variable influence from cryptic Archean rocks in the lower crustal melting zone of the Bangweulu Block. U-Pb analyses of inherited zircon cores in magmatic zircon in these granitoid rocks, directly confirm the presence of this reworked cryptic Archean basement of the Bangweulu Craton. The age data confirm previously proposed tectonic models for the Mesoproterozoic evolution of central Africa, refuting the presence of a continent-spanning Grenvillian-aged Orogen, including the Kibaran Belt, Irumide Belt and ChomaKalomo Block of central Africa and connecting with Mesoproterozoic terranes further south along the margins of the Kalahari Craton. The data clearly show that the Proterozoic tectonic evolution of the Bangweulu Craton, which became attached to the southern margin of the larger Congo Craton during the Mesoproterozoic, involved a series of distinct convergent orogenic episodes affecting and reworking its southern (passive) margin. The mismatch in timing of Mesoproterozoic orogenic activity along the Bangweulu Craton, compared to that on the margins of the Kalahari, is compatible with the notion that these continental fragments were not juxtaposed along these Mesoproterozoic belts and in their present-day relative positions at the time. Whether either of these central and southern African cratons did form part of Rodinia, however, remains a matter for debate.
\end{abstract}

INTRODUCTION

The Irumide Belt is an east-northeast-trending orogenic belt situated on the southern margin of the Bangweulu Block, part of the Congo Craton. It is composed of Paleoproterozoic basement and supracrustal units intruded by various granitoid suites. To the southwest, lithologies of the Irumide Belt are structurally overlain by Neopro-

* Tectonics Special Research Centre, Department of Applied Geology, Curtin University of Technology, GPO Box U1987, Perth, Western Australia 6845, Australia; (Present address: BDW, SRK Consulting, 10 Richardson Street, West Perth, Western Australia 6005, Australia, bdewaele@srk.com.au) Australia

** The Geological Survey of Western Australia, 100 Plain Street, East Perth, Western Australia 6004,

*** Geology Department, School of Mines, University of Zambia, P.O. BOX 32379, Lusaka, Zambia

$\$$ Geology Department, University of Namibia, Windhoek, Namibia

$\$ \$$ GEMOC Key Centre, Department of Earth and Planetary Sciences, Macquarie University, New South Wales 2109, Australia 
terozoic nappes of the Lufilian Belt (in the literature also referred to as Lufilian Arc, Katangan Belt or central African Copperbelt) and the Zambezi Belt (fig. 1A), making precise delineation of the southwestern margin of the Irumide Belt tenuous. Local tectonic windows through the Neoproterozoic cover occur south of the Mwembeshi Shear Zone (figs. 1A and B) and indicate the presence of Mesoproterozoic units below the Zambezi Belt (Mpande Gneiss, $1.10 \mathrm{Ga}$, Hanson and others, 1988; Munali Granite, $1.09 \mathrm{Ga}$, Katongo and others, 2004), but their relation to the Irumide Belt proper remains unclear. Farther southwest, the Choma-Kalomo Block comprises Mesoproterozoic granitoids dated at 1.38 and $1.18 \mathrm{Ga}$ (Bulambo and others, 2004, 2006). To the northeast, Irumide structures are truncated by Neoproterozoic shear zones within the reactivated Paleoproterozoic Ubendian Belt (fig. 1B). Possible correlations between units in the Irumide Belt and lithologies farther southeast are hampered by the intervening Phanerozoic Karoo graben (fig. 1B) and, until recently, a lack of reliable geochronological data, especially to the southeast of the graben.

The Irumide Belt was first described by Ackermann $(1950,1960)$ as a fold and thrust belt, which showed a marked divergence from northwest-directed thrusting towards a foreland in the Bangweulu Block to southeast-directed back-thrusting towards the terranes of the Zambezi and Southern Irumide belts that were overprinted during subsequent Neoproterozoic tectonism. Ackermann $(1950,1960)$ recognized a crystalline basement, termed the Mkushi Gneiss, unconformably, and in places structurally, overlain by a metasedimentary succession of quartzite and metapelite called "die Muva". Later mapping revealed that the Mkushi Gneiss and the Muva were intruded by various granitoid plutons (Stillman, 1965), while the Muva itself comprised amphibolite- and greenschist-facies components. Daly and Unrug (1982) proposed the name Muva Supergroup to include all pre-Neoproterozoic sedimentary successions of northern Zambia. The terms Kanona Group and Manshya River Group were formalized by De Waele and Mapani (2002) to denote the deformed metasedimentary sequences of the Muva Supergroup within the southwestern and northeastern Irumide Belt respectively (see fig. 1B). The Irumide Belt was interpreted by Daly (ms, 1986) as a thin-skinned fold- and thrust belt, with northwest-directed tectonic transport, in which extensive crustal shortening was accommodated through tight folding and thrusting within the Irumide Belt and along lateral shear zones within the Ubendian Belt. Back-thrusting described by Ackermann (1950) in the southwestern Irumide Belt was interpreted by Daly (ms, 1986) as a pop-up structure within an overall northwestdirected tectonic regime. The absence of reliable geochronological constraints has for a long time hampered a detailed understanding of the geological evolution of the Irumide Belt. Neither the age of basement units, the depositional age and detrital provenance of the Kanona and Manshya River groups, the age of granitoid rocks intruding both basement and supracrustals, and the timing of tectonism were constrained until recent years (see Ph. D. dissertation of De Waele, 2005).

This contribution presents zircon U-Pb SHRIMP and Laser Ablation Lu-Hf data on granitoid magmatism in the Irumide Belt, and ages of zircon xenocrysts within these intrusions, which constrain the age of cryptic basement components within the Irumide Belt. The geochronological database reported here refines and supersedes preliminary ages reported in De Waele and others (2003, 2006a, 2006b) and Johnson and others (2005b). Detailed descriptions of the samples and treatment of the data are also reported in the $\mathrm{Ph}$. D. dissertation of De Waele (2005).

GEOLOGY AND PREVIOUS GEOCHRONOLOGY OF THE IRUMIDE BELT

The following major lithotectonic components have been recognized within the Irumide Belt: (a) a gneissic granitoid basement complex that has yielded Paleoproterozoic (2.05-1.96 Ga) and subordinate Archean (2.7 Ga) crystallization ages; (b) the 1.94 to 1.66 Ga Kanona and Manshya River Group metasedimentary succession of quartzite 


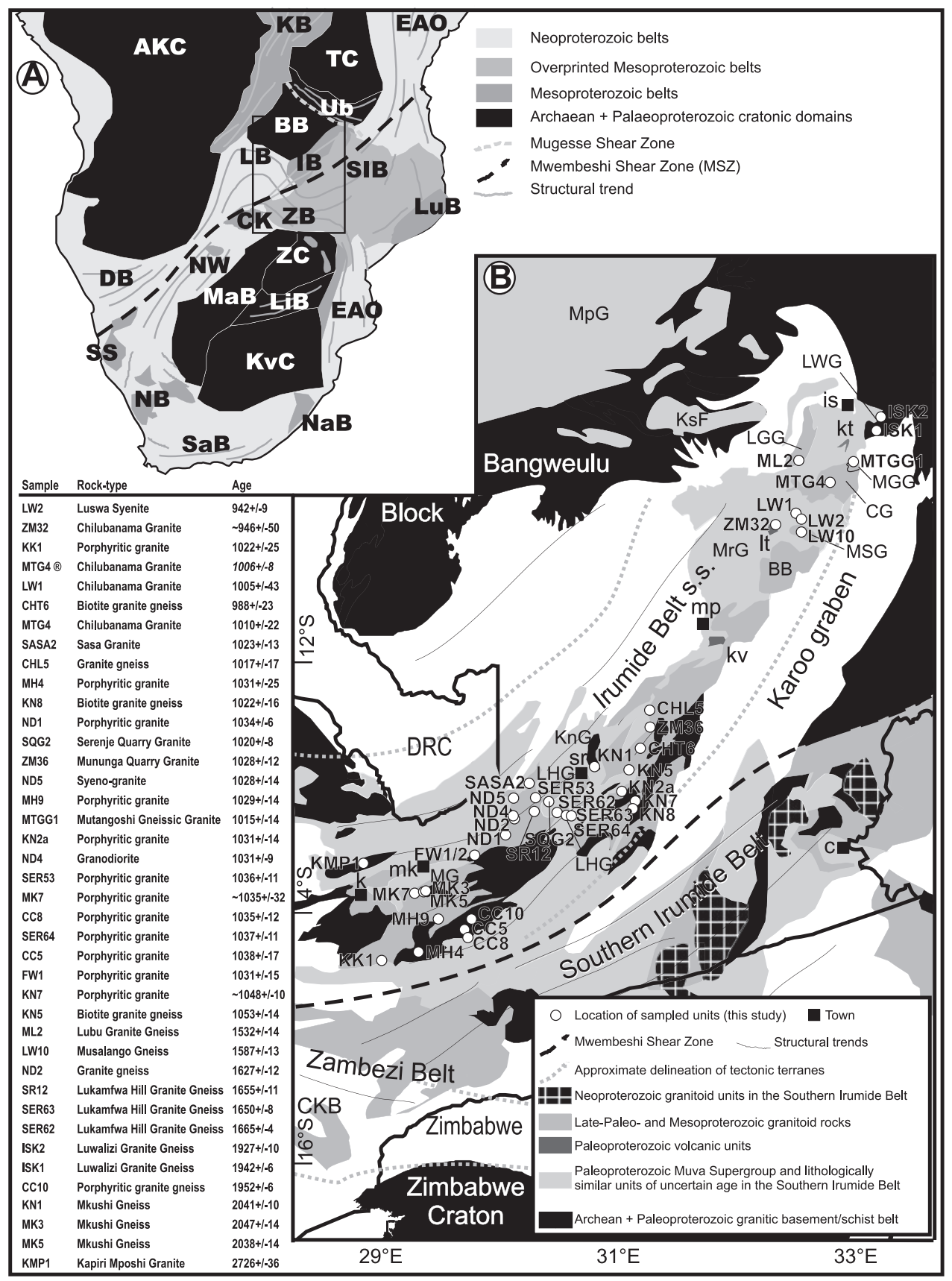

Fig. 1. (A) Simplified regional tectonic map of central and southern Africa (modified after Hanson, 2003) showing the approximate location of figure 1(B); AKC=Angola-Kasai Craton; $\mathrm{BB}=$ Bangweulu Block; $\mathrm{CK}=$ Choma-Kalomo Block; $\mathrm{DB}=$ Damara Belt; $\mathrm{EAO}=$ East African Orogen; $\mathrm{IB}=$ Irumide Belt; $\mathrm{KB}=\mathrm{Kibaran}$ Belt; $\mathrm{KvC}=$ Kaapvaal Craton; $\mathrm{LB}=$ Lufilian Belt; $\mathrm{LiB}=\mathrm{Limpopo}$ Belt; $\mathrm{LuB}=$ foreland to the Lurio Belt; $\mathrm{MaB}=$ Magondi Belt; $\mathrm{NaB}=$ Natal Belt; $\mathrm{NB}=$ Namaqua Belt; NW=northwest Botswana Rift; $\mathrm{SIB}=$ Southern Irumide Belt; $\mathrm{SS}=$ Sinclair Sequence; $\mathrm{SaB}=$ Saldania Belt; $\mathrm{TC}=$ Tanzania Craton; $\mathrm{Ub}=$ Ubendian-Usagaran Belt; $\mathrm{ZB}=$ Zambezi Belt; ZC=Zimbabwe Craton. (B) Regional geological map of central Zambia showing location and age of samples in this study; abbreviations used: $\mathrm{BB}=$ Bemba Batholith (also known as Lufila Granite); $\mathrm{CG}=$ Chilubanama Granite; $\mathrm{KnG}=$ Kanona Group; $\mathrm{KsF}=$ Kasama Formation; LGG=Lubu Granite Gneiss; $\mathrm{LHG}=$ Lukamfwa Hill Granite Gneiss; LWG=Luwalizi Granite Gneiss; MG=Mkushi Gneiss; MpG=Mporokoso Group; $\mathrm{MrG}=$ Manshya River Group; kv=Katibunga Volcanic rocks; lt=Luswa Tuff; kt=Kachinga Tuff. Location names: $\mathrm{c}=$ Chipata; is $=$ Isoka; $\mathrm{k}=$ Kapiri Mposhi; $\mathrm{mk}=$ Mkushi; $\mathrm{mp}=$ Mpika; $\mathrm{s}=$ Serenje. The sample denoted with ${ }^{\circledR}$ reflects the age of low Th/U metamorphic zircon rims. 
and metapelite, which forms part of the regionally defined Muva Supergroup of Daly and Unrug (1982), and in which volcanic units have been dated at between 1.88 and $1.85 \mathrm{Ga}$; (c) 1.66 and 1.52 Ga granitoid plutons that intrude the Kanona and Manshya River groups; and (d) 1.05 and 0.95 granitoid bodies emplaced during, and immediately following the Irumide Orogeny. These are described in more detail in the following sections.

\section{Basement Units to the Muva Supergroup}

Granitic basement identified in the Irumide Belt is referred to as the Mkushi Gneiss in the southwest (De Waele and Mapani, 2002), and the Mwambwa River, Lubu and Luwalizi Granite Gneiss in the northeast (MSG and LGG on fig. 1B, Daly, 1995a, 1995b). Similar basement units described further northeast, and referred to as the Mulungwizi Gneiss (Van Tuijl and Verhoog, 1995; Verhoog and Van Tuijl, 1995a, 1995b) and the Nyika Granite (Dodson and others, 1975; Vrána and others, 2004) form part of the Paleoproterozoic Ubendian Belt and are not further described here. The Mkushi Gneiss comprises a complex of deformed granitoid gneisses occurring throughout much of the southwestern part of the Irumide Belt (Rainaud and others, 2002, 2003, 2005). Previously reported zircon U-Pb dating has shown that similar basement extends westwards underneath the Lufilian Belt within the Domes region of the Copperbelt. This basement comprises granitic rocks dated between 2.05 and 1.97 $\mathrm{Ga}$ and a volcano-plutonic suite with crystallization ages between 1.88 and $1.87 \mathrm{Ga}$ (Ngoyi and others, 1991; John and others, 1999; John, ms, 2001; Key and others, 2001; Rainaud and others, 2002, 2003, 2005). The Mkushi Gneiss itself is best exposed in a large quarry south of Mkushi (location labelled MG on fig. 1B), where it is biotitebearing, and intruded by pink biotite granite and numerous large cupriferous aplites. Even though the entire complex is strongly deformed, the expression of foliation ranges from weak to extremely strong. Heterogeneous strain distribution led to the occurrence of gradational zones from mylonitic gneiss to coarse phenocrystic granite within the same gneiss body, which prompted many previous authors to include all granitoids into the Mkushi Gneiss (for example Stillman, 1965; Smith, 1966; Cvetcovic, 1973). Locally, however, sharp intrusive contacts can be observed between largely undeformed porphyritic granitoids and the Mkushi Gneiss, and intrusive contacts between phenocrystic granite and the metasedimentary successions, showing that at least some of the granitoid units post-date the Mkushi Gneiss Complex and the deposition of the regionally defined Muva Supergroup.

The basement underneath the northeastern Irumide Belt comprises deformed granitic gneiss units, which were called the Lubu and Luwalizi Granite Gneiss (LGG and LWG respectively on fig. 1B, Daly, ms, 1986). The Luwalizi Granite Gneiss yielded zircon U-Pb SHRIMP crystallization ages between 1.94 and $1.93 \mathrm{Ga}$, but the Lubu Granite Gneiss yielded a much younger age of 1.55 Ga (De Waele and others, 2003, 2006a, 2006b) indicating it post-dates deposition of at least part of the Muva Supergroup and does not form part of the basement.

\section{Metasedimentary Successions of the Irumide Belt}

The Muva Supergroup extends from the northwest foreland of the Irumide Belt (Bangweulu Block) into the Irumide Belt itself. Within the Irumide Belt, the Muva Supergroup is polydeformed and metamorphosed, whereas in the foreland it is little deformed and virtually unmetamorphosed. Within the Irumide Belt, the stratigraphy has been simplified into two groups, the Kanona Group in the southwest and the Manshya River Group in the northwest (KnG and MrG respectively on fig. 1B, De Waele and Mapani, 2002; De Waele and Fitzsimons, 2007). In unmetamorphosed foreland and lower-grade parts within the northeastern Irumide Belt, the succession has been described as a sequence of alternating quartzites and pelites, the purity, 
sorting and primary fabrics of which indicate shallow marine to coastal deposition (Daly and Unrug, 1982). Various conglomeratic units occur, but never over a wide enough area to allow them to be used as marker beds. The thickness of the sedimentary pile in the foreland has been estimated to be up to 6 kilometers. In the Irumide Belt itself this increases to 12 kilometers (Mapani, 1999) due to tectonic duplication during Irumide tectonics. High metamorphic grades, especially towards the internal portions of the Irumide Belt to the southeast, are locally expressed by anatexis of the metasedimentary pile, giving rise to formation of migmatite and paragneiss. Some isolated small occurrences of conformable rhyolitic units have been reported within the Manshya River Group in the northeast (Mosley and Marten, 1979; Daly, 1995b; Sykes, 1995). The most extensive occurrence, the Kachinga tuff, is located south of Isoka (kt on fig. 1B) and comprises a thinly layered sequence of recrystallized rhyolitic tuff of an estimated thickness of 300 meters. Smaller occurrences include the Luswa River Tuff southwest of Chinsali and the Katibunga volcanics southeast of Mpika (lt and kv on fig. 1B). Samples collected from these three locations yielded zircon U-Pb SHRIMP ages between 1.88 and $1.85 \mathrm{Ga}$, dating the age of deposition at these stratigraphic levels (De Waele, ms, 2005; De Waele and Fitzsimons, 2007).

\section{Post-Muva Supergroup Granitoid Intrusions}

Various granitoid bodies, now recognized to post-date deposition of the Muva Supergroup, have been described from the northern Irumide Belt. In the past, these were erroneously attributed to the Paleoproterozoic basement complex. These granitoid bodies can be distinguished into pre-tectonic and late-tectonic suites with respect to the Irumide Orogeny, based on internal fabrics, intrusive relationships, and the occurrence and character of xenoliths they contain. Pre-Irumide bodies are strongly deformed into granite gneiss, with or without feldspar augen, whereas late-Irumide bodies are only weakly foliated. A large proportion of granite bodies, both pre- and late-Irumide, are megacrystic with microcline phenocrysts of several tens of centimeters.

\section{Pre-Irumide Granitoid Bodies Intruding the Basement and Muva Supergroup}

Pre-Irumide granitoid bodies that post-dated deposition of the Muva Supergroup are known as the Mutangoshi Gneissic Granite and Musalango Gneiss in the northeast (MSG on fig. 1B), and the Lukamfwa Hill Granite Gneiss in the southwest (LHG on fig. 1B). These consist of strongly foliated, in places migmatitic, biotite granite gneiss, which often contain xenoliths of mafic composition and are cut by various mafic dikes and pegmatites. Coarser varieties contain K-feldspar phenocrysts, and are imparted with a strong biotite foliation, which predominantly affects the matrix, but in places gives rise to a distinct augen texture. Four samples from the Lukamfwa Hill Granite suite yielded preliminary zircon U-Pb SHRIMP crystallization ages between 1.66 and $1.63 \mathrm{Ga}$ (De Waele and others, 2003, 2006a, 2006b; De Waele, ms, 2005), but two strongly tectonized samples of the Mutangoshi Gneissic Granite yielded crystallization ages of between 1.98 and $1.95 \mathrm{Ga}$, with growth of metamorphic zircon between 1.06 and $1.03 \mathrm{Ga}$ (De Waele, ms, 2005; De Waele and others, 2006a). A previously reported whole-rock Rb-Sr date for the Mutangoshi Gneissic Granite of $1407 \pm 33$ Ma reported by Daly (ms, 1986) is therefore reinterpreted to reflect a partially reset and mixed age to which no geological interpretation can be ascribed. The Musalango Gneiss yielded a preliminary zircon U-Pb crystallization age of $1.61 \mathrm{Ga}$ (recalculated here as $1587 \pm 13$ $\mathrm{Ma}$ ) and is therefore slightly younger than the Lukamfwa Hill Granite Gneiss of the southwestern Irumide Belt (De Waele and others, 2006a). The Lubu Granite Gneiss, previously considered a part of the Paleoproterozoic basement (Daly, ms, 1986), yielded a preliminary crystallization age of $1.55 \mathrm{Ga}$ (De Waele and others, 2003, 2006a, $2006 \mathrm{~b})$, recalculated here to $1532 \pm 14 \mathrm{Ma}$. 


\section{Irumide Granitoid Bodies}

The 1.05 to 0.95 Ga Irumide granitoid rocks form batholiths and smaller plutonic bodies, composed of coarse biotite granite, leucogranite and syeno-granite. They show evidence of extensive crustal assimilation, and contain numerous mafic and felsic inclusions, xenoliths and large rafts of country rock, including metasedimentary rocks which could have been derived from the Muva Supergroup. In the northeast, the young granites are known as the Bemba Batholith [or Lufila granite, (Daly, ms, 1986), marked BB on fig. 1B], the Kaunga Granite, and the Chilubanama Granite [or Grey Granite, (Daly, ms, 1986), marked CG on fig. 1B]. In the southwest, the granites have often been ascribed to the Mkushi Gneiss Complex, and are represented by voluminous intrusions of porphyritic granite with clear intrusive relationship with the Mkushi Gneiss, the Muva Supergroup and the Lukamfwa Hill Granite Gneiss. Preliminary age data were reported by De Waele and others (2003, 2006a, 2006b) and bracket magmatism between 1.05 and $0.95 \mathrm{Ga}$.

U-Pb SHRIMP METHODOLOGY

Granitoid lithologies that range from undeformed to foliated were sampled from across the belt (refer for zircon characteristics to table 1, and for sample localities to fig. 1B and table 2). Zircon was extracted using conventional crushing and sieving over a $300 \mu \mathrm{m}$ mesh, followed by density and magnetic separation. Individual zircon grains were hand picked under binocular microscope, and mounted together with CZ3 zircon standard in epoxy resin mount, polished and gold coated. The mount was imaged using transmitted, reflected and cathodo-luminescence (CL) imaging, and analysis spots selected (Representative CL and optical images are presented in fig. 2). The mount was loaded into the SHRIMP [an acronym of "Sensitive High (mass) Resolution Ion MicroProbe"] sample lock 24 hours before actual analysis, and pumped to high vacuum to minimize hydrogen interferences. Each analyzed spot was rastered with the primary ion beam for 90 to 120 seconds prior to analysis, to reduce surface-related contamination. Analyses were conducted during several sessions, with standards interspersed at a one to four ratio, approximately one every 90 minutes. Count/delay times (seconds) on each mass were $\mathrm{Zr}_{2} \mathrm{O}(2 / 4),{ }^{204} \mathrm{~Pb}(10 / 1)$, background $(10 / 1),{ }^{206} \mathrm{~Pb}(10 / 1),{ }^{207} \mathrm{~Pb}(30 / 1),{ }^{208} \mathrm{~Pb}(10 / 1),{ }^{238} \mathrm{U}(5 / 3), \mathrm{ThO}_{2}(5 / 2)$ and $\mathrm{UO}_{2}(2 / 2)$. Data were reduced using SQUID and ISOPLOT (Ludwig, 2001a, 2001b). Corrections for common lead were based on measured ${ }^{204} \mathrm{~Pb}$, and assuming an isotopic composition appropriate for the age of the sample (Stacey and Kramers, 1975). Common $\mathrm{Pb}\left(\mathrm{or} \mathrm{Pb}_{\mathrm{c}}\right)$ is reported as the proportion of non-radiogenic ${ }^{206} \mathrm{~Pb}$ in total ${ }^{206} \mathrm{~Pb}\left(f_{206}\right)$. The standard used was a gem zircon called CZ3, for which a uranium content of $551 \mathrm{ppm}$ and age of 564 Ma were assumed, corresponding to a ${ }^{206} \mathrm{~Pb} /{ }^{238} \mathrm{U}$ ratio of 0.09143 (Pidgeon and others, 1994). The error on the decay constant has been propagated in the age calculations, but the error on the standard was not included where analyses were conducted in a single session. Where analyses obtained during several sessions were combined, however, the standard error was added in quadrature to the data. Standard errors are quoted in the data tables for each session. All ages are reported at the $2 \sigma$ (or 95\%) confidence level, but spot ages in the tables are reported at $1 \sigma$ level. The data are shown in figure 3 and figures 5 through 12 as Tera-Wasserburg plots, with error crosses at $2 \sigma$ confidence level. Analyses indicated in gray were not included in the calculation of a pooled age.

\section{Lu-Hf METHODOLOGY}

Hf-isotope analyses were conducted on separate zircon grain mounts, that is on zircons from the same separates as those used for SHRIMP analysis. The setup comprised a $213 \mathrm{~nm} \mathrm{Nd:YAG} \mathrm{laser} \mathrm{microprobe} \mathrm{attached} \mathrm{to} \mathrm{a} \mathrm{Nu} \mathrm{Plasma} \mathrm{multi-collector}$ 


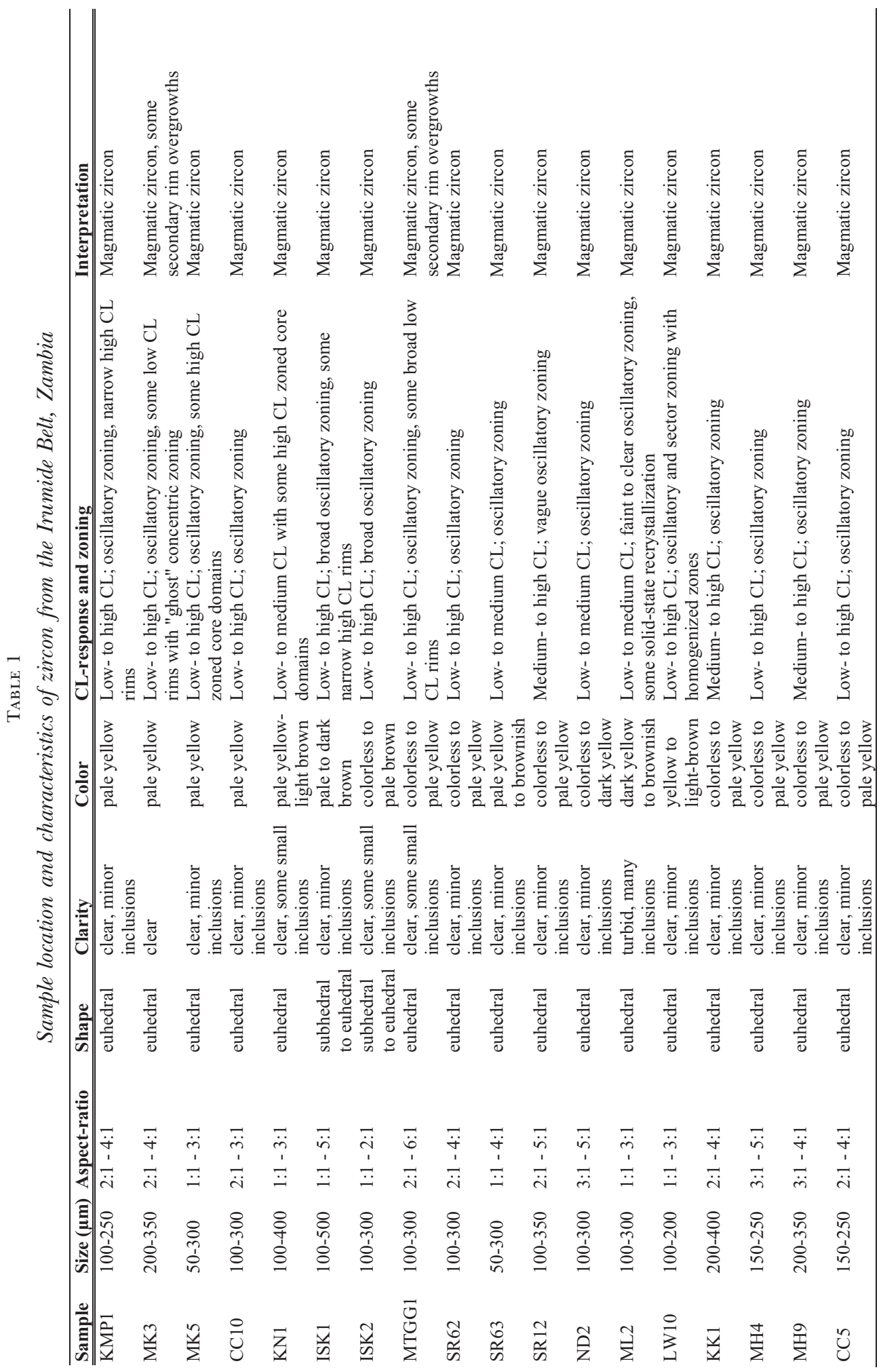




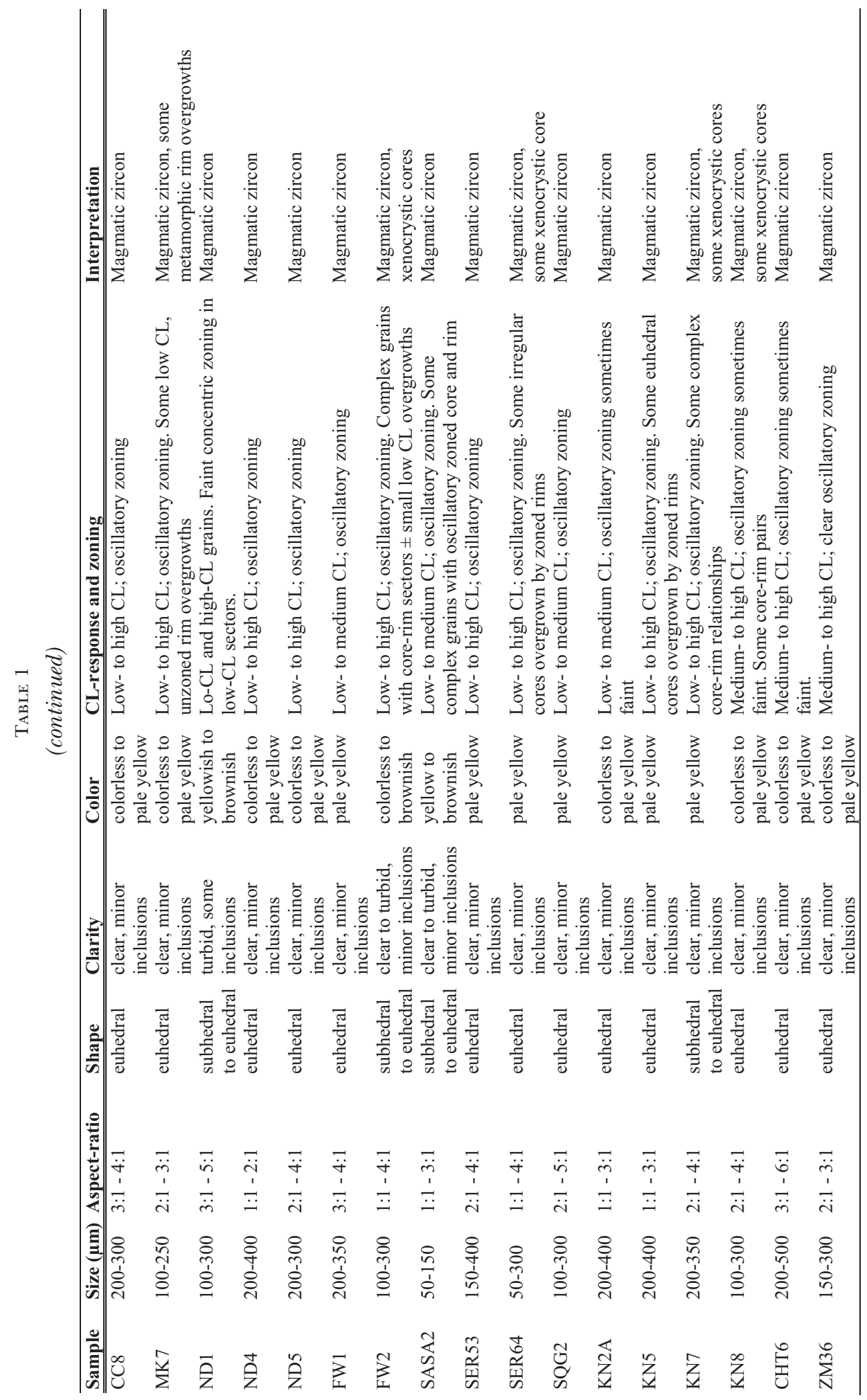




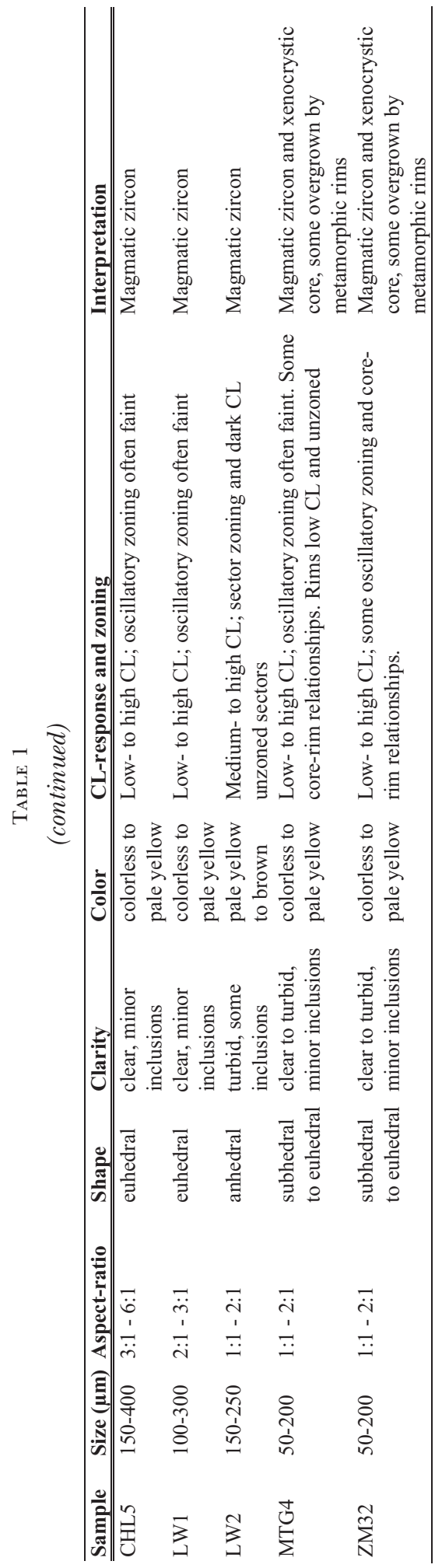


TABle 2

Sample locations and zircon U-Pb SHRIMP data

\begin{tabular}{|c|c|c|c|c|c|c|c|c|}
\hline Spot Name & $\begin{array}{l}f_{206} \\
(\%)\end{array}$ & $\begin{array}{c}\mathrm{U} \\
(\mathrm{ppm})\end{array}$ & $\begin{array}{c}\text { Th } \\
(\mathbf{p p m})\end{array}$ & $\mathbf{T h} / \mathbf{U}$ & $\begin{array}{c}{ }^{207} \mathrm{~Pb}^{/ 206} \mathrm{~Pb} \\
( \pm 1 \sigma)\end{array}$ & $\begin{array}{c}{ }^{206} \mathrm{~Pb}^{1238} \mathrm{U} \\
( \pm 1 \sigma)\end{array}$ & $\begin{array}{c}{ }^{207} \mathrm{~Pb}^{1206} \mathrm{~Pb} \text { Age } \\
( \pm 1 \sigma \mathrm{Ma})\end{array}$ & $\mathrm{C}(\%)$ \\
\hline \multicolumn{9}{|c|}{ Sample KMP1 : Kapiri Mposhi Granite Gneiss (13.7458 S - 28.6799 E) } \\
\hline \multicolumn{9}{|c|}{ Analyses conducted during a single session. $10 \mathrm{CZ} 3$ standard analyses yielded a $2 \sigma$ error of the mean of $0.36 \%$} \\
\hline KMP1-1 & 0.160 & 361 & 109 & 0.31 & $0.18520 \pm 0.00058$ & $0.49807 \pm 0.00196$ & $2700.0 \pm 5.1$ & 96.5 \\
\hline KMP1-2 & 5.328 & 300 & 192 & 0.66 & $0.23768 \pm 0.02128$ & $0.60223 \pm 0.06121$ & $3104.5 \pm 142.7$ & 97.9 \\
\hline KMP1-1r & 1.134 & 559 & 358 & 0.66 & $0.18360 \pm 0.00145$ & $0.45528 \pm 0.00159$ & $2685.7 \pm 13.0$ & 90.1 \\
\hline KMP1-3 & 1.946 & 714 & 440 & 0.64 & $0.18677 \pm 0.00507$ & $0.44983 \pm 0.00260$ & $2713.9 \pm 44.7$ & 88.2 \\
\hline KMP1-4 & 0.087 & 597 & 416 & 0.72 & $0.18951 \pm 0.00043$ & $0.53337 \pm 0.00163$ & $2737.9 \pm 3.7$ & 100.6 \\
\hline KMP1-5 & 0.421 & 665 & 226 & 0.35 & $0.18727 \pm 0.00067$ & $0.40214 \pm 0.00154$ & $2718.3 \pm 5.9$ & 80.2 \\
\hline \multicolumn{9}{|c|}{ Sample MK3 : Mkushi Gneiss (13.9367 S - 29.1490 E) } \\
\hline \multicolumn{9}{|c|}{ Analyses conducted during a single session. $10 \mathrm{CZ} 3$ standard analyses yielded a $2 \sigma$ error of the mean of $0.36 \%$} \\
\hline MK3-1 & 0.038 & 228 & 194 & 0.88 & $0.12646 \pm 0.00066$ & $0.36575 \pm 0.00188$ & $2049.3 \pm 9.2$ & 98.1 \\
\hline MK3-2r & 0.024 & 770 & 56 & 0.08 & $0.12404 \pm 0.00047$ & $0.35671 \pm 0.00101$ & $2015.1 \pm 6.7$ & 97.6 \\
\hline MK3-3r & 0.002 & 891 & 88 & 0.10 & $0.12515 \pm 0.00034$ & $0.36597 \pm 0.00100$ & $2031.0 \pm 4.8$ & 99.0 \\
\hline MK3-4r & 0.000 & 137 & 122 & 0.91 & $0.12605 \pm 0.00082$ & $0.36105 \pm 0.00244$ & $2043.6 \pm 11.6$ & 97.2 \\
\hline MK3-4c & 0.089 & 466 & 411 & 0.91 & $0.12560 \pm 0.00054$ & $0.32834 \pm 0.00133$ & $2037.3 \pm 7.7$ & 89.8 \\
\hline
\end{tabular}

Sample MK5 : Mkushi Gneiss (13.9432 S - 29.1490 E)

Analyses conducted during two sessions

Session 1 : 10 CZ3 standard analyses yielded a $2 \sigma$ error of the mean of $0.98 \%$

Session 2 : $10 \mathrm{CZ} 3$ standard analyses yielded a $2 \sigma$ error of the mean of $0.36 \%$

\begin{tabular}{|c|c|c|c|c|c|c|c|c|}
\hline $1 \mathrm{MK} 5-1$ & 0.167 & 569 & 351 & 0.64 & $0.12591 \pm 0.00064$ & $0.35801 \pm 0.00496$ & $2041.6 \pm 9.0$ & 96.6 \\
\hline $1 \mathrm{MK} 5-2$ & 0.013 & 681 & 369 & 0.56 & $0.12508 \pm 0.00027$ & $0.36034 \pm 0.00460$ & $2029.9 \pm 3.9$ & 97.7 \\
\hline 1MK5-3 & 0.712 & 687 & 408 & 0.61 & $0.12480 \pm 0.00065$ & $0.31352 \pm 0.00400$ & $2026.0 \pm 9.2$ & 86.8 \\
\hline 2MK5-4 & 0.090 & 804 & 367 & 0.47 & $0.12116 \pm 0.00048$ & $0.32508 \pm 0.00095$ & $1973.4 \pm 7.1$ & 91.9 \\
\hline $2 \mathrm{MK} 5-5 \mathrm{r}$ & 0.034 & 587 & 329 & 0.58 & $0.12669 \pm 0.00044$ & $0.36302 \pm 0.00221$ & $2052.5 \pm 6.2$ & 97.3 \\
\hline 2MK5-6 & 0.050 & 505 & 409 & 0.84 & $0.12639 \pm 0.00129$ & $0.37341 \pm 0.00170$ & $2048.3 \pm 18.0$ & 99.9 \\
\hline 2MK5-7 & 0.280 & 224 & 139 & 0.64 & $0.12665 \pm 0.00079$ & $0.35236 \pm 0.00197$ & $2051.9 \pm 11.0$ & 94.8 \\
\hline \multicolumn{9}{|c|}{ Sample CC10 : Mkushi Gneiss (14.3932 S - 29.6785 E) } \\
\hline \multicolumn{9}{|c|}{ Analyses conducted during a single session. $16 \mathrm{CZ} 3$ standard analyses yielded a $2 \sigma$ error of the mean of $2.10 \%$} \\
\hline CC10-1.1 & 0.000 & 693 & 612 & 0.91 & $0.12058 \pm 0.00034$ & $0.37259 \pm 0.01464$ & $1964.9 \pm 5.1$ & 103.9 \\
\hline CC10-2.1 & 0.851 & 460 & 343 & 0.77 & $0.11507 \pm 0.00428$ & $0.30681 \pm 0.01212$ & $1881.0 \pm 67.0$ & 91.7 \\
\hline CC10-3.1 & 0.163 & 514 & 296 & 0.60 & $0.11950 \pm 0.00046$ & $0.35108 \pm 0.01380$ & $1948.7 \pm 6.9$ & 99.5 \\
\hline CC10-4.1 & 0.426 & 167 & 121 & 0.75 & $0.11864 \pm 0.00203$ & $0.34657 \pm 0.01371$ & $1935.9 \pm 30.6$ & 99.1 \\
\hline CC10-5.1 & 0.051 & 403 & 283 & 0.72 & $0.11942 \pm 0.00050$ & $0.33052 \pm 0.01300$ & $1947.6 \pm 7.5$ & 94.5 \\
\hline CC10-6.1 & 0.005 & 390 & 273 & 0.72 & $0.11898 \pm 0.00046$ & $0.33719 \pm 0.01326$ & $1940.9 \pm 6.9$ & 96.5 \\
\hline
\end{tabular}

Sample KN1 : Mkushi Gneiss (12.9652 S - 30.6258 E)

Analyses conducted during a single session. $12 \mathrm{CZ} 3$ standard analyses yielded a $2 \sigma$ error of the mean of $1.56 \%$

\begin{tabular}{lcccccccc}
\hline KN1-1 & 0.002 & 335 & 198 & 0.61 & $0.12935 \pm 0.00067$ & $0.30750 \pm 0.00816$ & $2089.1 \pm 9.1$ \\
KN1-2 & 0.005 & 268 & 68 & 0.26 & $0.12518 \pm 0.00068$ & $0.33974 \pm 0.00833$ & $2031.3 \pm 9.6$ & 92.7 \\
KN1-3 & 0.041 & 307 & 112 & 0.38 & $0.12539 \pm 0.00056$ & $0.36288 \pm 0.00896$ & $2034.3 \pm 7.9$ \\
KN1-4 & 0.000 & 440 & 215 & 0.51 & $0.12547 \pm 0.00045$ & $0.35235 \pm 0.00855$ & $2035.5 \pm 6.4$ \\
KN1-5 & 0.000 & 414 & 200 & 0.50 & $0.12570 \pm 0.00044$ & $0.37025 \pm 0.00899$ & $2038.7 \pm 6.1$ & 95.1 \\
KN1-6 & 0.007 & 386 & 223 & 0.60 & $0.12647 \pm 0.00046$ & $0.37061 \pm 0.00901$ & $2049.4 \pm 6.4$ & 99.6 \\
\hline
\end{tabular}

Sample ISK1 : Luwalizi Granite Gneiss (10.2443 S - 32.8449 E)

Analyses conducted during a single session. $15 \mathrm{CZ} 3$ standard analyses yielded a $2 \sigma$ error of the mean of $0.51 \%$

\begin{tabular}{lcccccccc}
\hline ISK1-1 & 0.029 & 253 & 179 & 0.73 & $0.11875 \pm 0.00068$ & $0.33595 \pm 0.00342$ & $1937.5 \pm 10.2$ \\
ISK1-2 & 0.028 & 2669 & 110 & 0.04 & $0.11800 \pm 0.00055$ & $0.36163 \pm 0.00369$ & $1926.1 \pm 8.3$ \\
ISK1-3 & 0.855 & 93 & 175 & 1.93 & $0.11577 \pm 0.00191$ & $0.34508 \pm 0.00413$ & $1892.0 \pm 29.8$ & 103.3 \\
ISK1-4 & 0.077 & 565 & 110 & 0.20 & $0.11893 \pm 0.00050$ & $0.34189 \pm 0.00322$ & $1940.1 \pm 7.6$ \\
ISK1-5 & 0.061 & 521 & 109 & 0.22 & $0.11895 \pm 0.00042$ & $0.34748 \pm 0.00325$ & $1940.6 \pm 6.4$ \\
ISK1-6 & 0.212 & 945 & 169 & 0.19 & $0.11838 \pm 0.00110$ & $0.32209 \pm 0.00634$ & $1931.9 \pm 16.7$ & 97.7 \\
ISK1-7 & 0.000 & 504 & 204 & 0.42 & $0.11916 \pm 0.00038$ & $0.34803 \pm 0.00340$ & $1943.6 \pm 5.7$ & 93.2 \\
ISK1-8 & 0.060 & 396 & 156 & 0.41 & $0.11917 \pm 0.00050$ & $0.33942 \pm 0.00345$ & $1943.8 \pm 7.5$ & 96.9 \\
ISK1-9 & 0.194 & 785 & 595 & 0.78 & $0.12008 \pm 0.00122$ & $0.34865 \pm 0.00318$ & $1957.4 \pm 18.2$ & 98.5 \\
\hline
\end{tabular}

Sample ISK2 : Luwalizi Granite Gneiss (10.2030 S - 32.8094 E)

Analyses conducted during a single session. $15 \mathrm{CZ} 3$ standard analyses yielded a $2 \sigma$ error of the mean of $0.51 \%$

\begin{tabular}{lcccccccc}
\hline ISK2-1 & 0.688 & 103 & 29 & 0.29 & $0.11666 \pm 0.00146$ & $0.35250 \pm 0.00409$ & $1905.7 \pm 22.5$ & 102.1 \\
ISK2-2 & 0.181 & 300 & 391 & 1.34 & $0.11926 \pm 0.00065$ & $0.30654 \pm 0.00299$ & $1945.2 \pm 9.8$ & 88.6 \\
ISK2-3 & 0.071 & 502 & 308 & 0.63 & $0.11781 \pm 0.00041$ & $0.33415 \pm 0.00310$ & $1923.3 \pm 6.2$ & 96.6 \\
ISK2-4 & 0.000 & 949 & 29 & 0.03 & $0.11919 \pm 0.00135$ & $0.35460 \pm 0.01018$ & $1944.2 \pm 20.2$ & 100.6 \\
ISK2-5 & 0.059 & 277 & 26 & 0.10 & $0.11879 \pm 0.00081$ & $0.34057 \pm 0.00336$ & $1938.1 \pm 12.2$ & 97.5 \\
\hline
\end{tabular}


TABLE 2

(continued)

\begin{tabular}{|c|c|c|c|c|c|c|c|c|}
\hline Spot Name & $\begin{array}{l}f_{206} \\
(\%)\end{array}$ & $\begin{array}{c}\mathrm{U} \\
(\mathbf{p p m})\end{array}$ & $\begin{array}{c}\text { Th } \\
(p p m)\end{array}$ & $\mathbf{T h} / \mathbf{U}$ & $\begin{array}{c}{ }^{207} \mathrm{~Pb}^{1206} \mathrm{~Pb} \\
( \pm 1 \sigma)\end{array}$ & $\begin{array}{c}{ }^{206} \mathrm{~Pb}^{/ 238} \mathrm{U} \\
( \pm 1 \sigma)\end{array}$ & $\begin{array}{c}{ }^{207} \mathrm{~Pb}^{1206} \mathrm{~Pb} \text { Age } \\
( \pm 1 \sigma \mathrm{Ma})\end{array}$ & $\mathrm{C}(\%)$ \\
\hline \multicolumn{9}{|c|}{ Sample MTGG1 : Mutangoshi Gneiss (10.6796 S - 32.5138 E) } \\
\hline \multicolumn{9}{|c|}{ Analyses conducted during a single session. $16 \mathrm{CZ} 3$ standard analyses yielded a $2 \sigma$ error of the mean of $0.81 \%$} \\
\hline MTGG1-1 & 0.025 & 1519 & 364 & 0.25 & $0.07177 \pm 0.00021$ & $0.15839 \pm 0.00260$ & $979.4 \pm 6.1$ & 96.8 \\
\hline MTGG1-2 & 0.068 & 540 & 197 & 0.38 & $0.07304 \pm 0.00042$ & $0.16698 \pm 0.00277$ & $1015.1 \pm 11.6$ & 98.1 \\
\hline MTGG1-3r & 0.063 & 1265 & 217 & 0.18 & $0.07279 \pm 0.00027$ & $0.16835 \pm 0.00276$ & $1008.0 \pm 7.5$ & 99.5 \\
\hline MTGG1-3c & 0.643 & 264 & 112 & 0.44 & $0.12030 \pm 0.00099$ & $0.34978 \pm 0.00586$ & $1960.7 \pm 14.7$ & 98.6 \\
\hline MTGG1-4c & 0.000 & 227 & 107 & 0.49 & $0.07443 \pm 0.00059$ & $0.16034 \pm 0.00274$ & $1053.1 \pm 16.0$ & 91.0 \\
\hline MTGG1-5 & 0.445 & 673 & 248 & 0.38 & $0.07256 \pm 0.00053$ & $0.15909 \pm 0.00270$ & $1001.7 \pm 14.8$ & 95.0 \\
\hline MTGG1-6c & 1.178 & 109 & 78 & 0.74 & $0.07366 \pm 0.00206$ & $0.16557 \pm 0.00294$ & $1032.1 \pm 56.7$ & 95.7 \\
\hline MTGG1-6r & 0.000 & 636 & 246 & 0.40 & $0.07369 \pm 0.00030$ & $0.17452 \pm 0.00289$ & $1033.0 \pm 8.2$ & 100.4 \\
\hline MTGG1-7c & 0.034 & 860 & 336 & 0.40 & $0.07352 \pm 0.00029$ & $0.17377 \pm 0.00286$ & $1028.3 \pm 8.0$ & 100.4 \\
\hline MTGG1-7r & 0.042 & 670 & 245 & 0.38 & $0.07349 \pm 0.00035$ & $0.17052 \pm 0.00282$ & $1027.5 \pm 9.7$ & 98.8 \\
\hline MTGG1-8 & 0.000 & 291 & 160 & 0.57 & $0.07318 \pm 0.00053$ & $0.17448 \pm 0.00294$ & $1019.0 \pm 14.7$ & 101.7 \\
\hline MTGG1-9 & 0.000 & 221 & 123 & 0.57 & $0.07380 \pm 0.00054$ & $0.17427 \pm 0.00296$ & $1036.1 \pm 14.7$ & 100.0 \\
\hline MTGG1-11 & 0.091 & 1130 & 590 & 0.54 & $0.07357 \pm 0.00026$ & $0.17951 \pm 0.00285$ & $1029.7 \pm 7.2$ & 103.3 \\
\hline MTGG1-12c & 0.041 & 364 & 686 & 1.95 & $0.11992 \pm 0.00042$ & $0.34793 \pm 0.00557$ & $1955.1 \pm 6.2$ & 98.4 \\
\hline MTGG1-13 & 2.942 & 2507 & 14 & 0.01 & $0.07241 \pm 0.00375$ & $0.17572 \pm 0.00281$ & $997.6 \pm 105.2$ & 104.4 \\
\hline MTGG1-14 & 0.091 & 1130 & 590 & 0.54 & $0.07357 \pm 0.00026$ & $0.17951 \pm 0.00285$ & $1029.7 \pm 7.2$ & 103.3 \\
\hline MTGG1-16c & 3.491 & 112 & 101 & 0.93 & $0.12224 \pm 0.00468$ & $0.35767 \pm 0.00608$ & $1989.2 \pm 68.1$ & 99.1 \\
\hline MTGG1-17 & 0.917 & 1329 & 533 & 0.41 & $0.07009 \pm 0.00113$ & $0.13773 \pm 0.00219$ & $930.9 \pm 33.2$ & 88.1 \\
\hline MTGG1-19 & 8.217 & 1708 & 40 & 0.02 & $0.07313 \pm 0.01482$ & $0.15059 \pm 0.00285$ & $1017.6 \pm 410.4$ & 87.5 \\
\hline MTGG1-20 & 0.091 & 1130 & 590 & 0.54 & $0.07357 \pm 0.00026$ & $0.17951 \pm 0.00285$ & $1029.7 \pm 7.2$ & 103.3 \\
\hline MTGG1-21 & 0.000 & 273 & 144 & 0.55 & $0.07477 \pm 0.00044$ & $0.18049 \pm 0.00292$ & $1062.4 \pm 11.8$ & 100.7 \\
\hline MTGG1-22 & 0.030 & 163 & 32 & 0.21 & $0.07279 \pm 0.00073$ & $0.17983 \pm 0.00301$ & $1008.0 \pm 20.5$ & 105.4 \\
\hline MTGG1-25c & 0.396 & 79 & 106 & 1.38 & $0.12150 \pm 0.00129$ & $0.35993 \pm 0.00624$ & $1978.3 \pm 18.9$ & 100.2 \\
\hline
\end{tabular}

Sample SER62c : Lukamfwa Hill Granite Gneiss (13.3121 S - 30.3506 E)

Analyses conducted during a single session. $16 \mathrm{CZ} 3$ standard analyses yielded a $2 \sigma$ error of the mean of $1.18 \%$

\begin{tabular}{lcccccccr}
\hline SER62-26c & 0.989 & 65 & 53 & 0.84 & $0.10376 \pm 0.00389$ & $0.30047 \pm 0.00779$ & $1692.5 \pm 69.2$ & 100.1 \\
SER62-26r & 0.047 & 770 & 452 & 0.61 & $0.10228 \pm 0.00028$ & $0.28729 \pm 0.00622$ & $1665.8 \pm 5.0$ & 97.7 \\
SER62-27 & 0.146 & 316 & 326 & 1.07 & $0.10317 \pm 0.00056$ & $0.29634 \pm 0.00664$ & $1681.8 \pm 10.0$ & 99.5 \\
SER62-29 & 0.246 & 100 & 81 & 0.84 & $0.10405 \pm 0.00191$ & $0.26150 \pm 0.00583$ & $1697.6 \pm 33.8$ & 88.2 \\
SER62-32 & 0.040 & 2804 & 2342 & 0.86 & $0.10179 \pm 0.00027$ & $0.28702 \pm 0.00617$ & $1657.1 \pm 4.9$ & 98.2 \\
SER62-36 & 0.368 & 588 & 214 & 0.38 & $0.10021 \pm 0.00041$ & $0.26012 \pm 0.00566$ & $1627.9 \pm 7.6$ & 91.6 \\
SER62-37 & 0.108 & 489 & 390 & 0.82 & $0.10257 \pm 0.00070$ & $0.30040 \pm 0.00676$ & $1671.1 \pm 12.6$ & 101.3 \\
SER62-40 & 0.433 & 112 & 125 & 1.16 & $0.10214 \pm 0.00179$ & $0.29847 \pm 0.00660$ & $1663.4 \pm 32.4$ & 101.2 \\
SER62-41c & 14.976 & 3965 & 2136 & 0.56 & $0.10197 \pm 0.00801$ & $0.10059 \pm 0.00293$ & $1660.2 \pm 145.4$ & 37.2 \\
SER62-41r & 0.542 & 148 & 98 & 0.69 & $0.10007 \pm 0.00198$ & $0.29888 \pm 0.00661$ & $1625.3 \pm 36.9$ & 103.7 \\
\hline
\end{tabular}

\section{Sample SER63 : Lukamfwa Hill Granite Gneiss (13.3319 S - 30.3927 E)}

Analyses conducted during a single session. $16 \mathrm{CZ} 3$ standard analyses yielded a $2 \sigma$ error of the mean of $1.18 \%$

\begin{tabular}{llllllllr}
\hline SER63-1 & 0.056 & 470 & 339 & 0.74 & $0.10201 \pm 0.00043$ & $0.29684 \pm 0.00644$ & $1661.0 \pm 7.8$ & 100.9 \\
SER63-2 & 0.183 & 320 & 259 & 0.84 & $0.10158 \pm 0.00092$ & $0.29511 \pm 0.00721$ & $1653.1 \pm 16.7$ & 100.8 \\
SER63-5 & 0.187 & 467 & 361 & 0.80 & $0.10014 \pm 0.00104$ & $0.29069 \pm 0.00628$ & $1626.6 \pm 19.3$ & 101.1 \\
SER63-6 & 0.062 & 378 & 309 & 0.84 & $0.10205 \pm 0.00074$ & $0.29462 \pm 0.00654$ & $1661.7 \pm 13.4$ & 100.2 \\
SER63-7 & 0.102 & 403 & 303 & 0.78 & $0.10176 \pm 0.00033$ & $0.29662 \pm 0.00642$ & $1656.6 \pm 6.0$ & 101.1 \\
SER63-8 & 0.359 & 331 & 356 & 1.11 & $0.10071 \pm 0.00214$ & $0.28016 \pm 0.00610$ & $1637.2 \pm 39.4$ & 97.2 \\
SER63-15 & 0.082 & 307 & 231 & 0.78 & $0.10193 \pm 0.00055$ & $0.29725 \pm 0.00678$ & $1659.6 \pm 10.0$ & 101.1 \\
SER63-19 & 0.178 & 280 & 211 & 0.78 & $0.09919 \pm 0.00125$ & $0.27687 \pm 0.00631$ & $1609.0 \pm 23.5$ & 97.9 \\
SER63-22 & 0.195 & 420 & 331 & 0.81 & $0.10099 \pm 0.00107$ & $0.29160 \pm 0.00644$ & $1642.3 \pm 19.7$ & 100.4 \\
SER63-29 & 0.057 & 501 & 288 & 0.59 & $0.10042 \pm 0.00070$ & $0.27852 \pm 0.00635$ & $1631.8 \pm 13.0$ & 97.1 \\
\hline
\end{tabular}

Sample SR12 : Lukamfwa Hill Granite Gneiss (13.2777 S - 30.1306 E)

Analyses conducted during a single session. $5 \mathrm{CZ3}$ standard analyses yielded a $2 \sigma$ error of the mean of $1.07 \%$

\begin{tabular}{|c|c|c|c|c|c|c|c|c|}
\hline$\overline{\text { SR12-1 }}$ & 0.215 & 141 & 110 & 0.81 & $0.10132 \pm 0.00092$ & $0.29495 \pm 0.00309$ & $1648.4 \pm 16.8$ & 101.1 \\
\hline SR12-2 & 0.000 & 198 & 204 & 1.07 & $0.10007 \pm 0.00155$ & $0.28166 \pm 0.00288$ & $1625.3 \pm 28.8$ & 98.4 \\
\hline SR12-3 & 0.136 & 139 & 84 & 0.63 & $0.09898 \pm 0.00095$ & $0.27508 \pm 0.00369$ & $1604.9 \pm 17.9$ & 97.6 \\
\hline SR12-4 & 0.067 & 179 & 138 & 0.79 & $0.10155 \pm 0.00076$ & $0.29499 \pm 0.00294$ & $1652.7 \pm 13.8$ & 100.8 \\
\hline SR12-8 & 0.823 & 138 & 142 & 1.06 & $0.10024 \pm 0.00376$ & $0.28273 \pm 0.00313$ & $1628.6 \pm 69.7$ & 98.6 \\
\hline SR12-9 & 0.093 & 103 & 117 & 1.17 & $0.10149 \pm 0.00121$ & $0.29459 \pm 0.00361$ & $1651.5 \pm 22.2$ & 100.8 \\
\hline SR12-10 & 0.088 & 222 & 256 & 1.19 & $0.10082 \pm 0.00066$ & $0.29085 \pm 0.00335$ & $1639.3 \pm 12.2$ & 100.4 \\
\hline \multicolumn{9}{|c|}{ 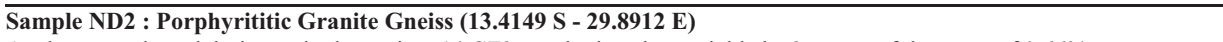 } \\
\hline \multicolumn{9}{|c|}{ Analyses conducted during a single session. $14 \mathrm{CZ3}$ standard analyses yielded a $2 \sigma$ error of the mean of $0.66 \%$} \\
\hline ND2-1 & 0.070 & 103 & 82 & 0.83 & $0.09989 \pm 0.00142$ & $0.28323 \pm 0.00373$ & $1622.0 \pm 26.5$ & 99.1 \\
\hline ND2-2 & 0.000 & 185 & 144 & 0.80 & $0.10018 \pm 0.00061$ & $0.29307 \pm 0.00349$ & $1627.4 \pm 11.3$ & 101.8 \\
\hline ND2-3 & 0.091 & 229 & 196 & 0.88 & $0.10031 \pm 0.00067$ & $0.28161 \pm 0.00337$ & $1629.7 \pm 12.5$ & 98.1 \\
\hline ND2-4 & 0.025 & 181 & 154 & 0.88 & $0.10110 \pm 0.00097$ & $0.28361 \pm 0.00661$ & $1644.5 \pm 17.8$ & 97.9 \\
\hline
\end{tabular}


TABLE 2

(continued)

\begin{tabular}{|c|c|c|c|c|c|c|c|c|}
\hline Spot Name & $\begin{array}{l}f_{206} \\
(\%)\end{array}$ & $\begin{array}{c}\mathbf{U} \\
(\mathbf{p p m})\end{array}$ & $\begin{array}{c}\text { Th } \\
(\mathbf{p p m})\end{array}$ & $\mathbf{T h} / \mathbf{U}$ & $\begin{array}{c}{ }^{207} \mathrm{~Pb}^{1206} \mathrm{~Pb} \\
( \pm 1 \sigma)\end{array}$ & $\begin{array}{c}{ }^{206} \mathrm{~Pb}^{1238} \mathrm{U} \\
( \pm 1 \sigma)\end{array}$ & $\begin{array}{c}{ }^{207} \mathrm{~Pb}^{1206} \mathrm{~Pb} \text { Age } \\
( \pm 1 \sigma \mathrm{Ma})\end{array}$ & $\mathrm{C}(\%)$ \\
\hline
\end{tabular}

Sample ML2 : Lubu Granite Gneiss (10.4796 S - 32.4367 E)

Analyses conducted during two sessions

Session $1: 5 \mathrm{CZ} 3$ standard analyses yielded a $2 \sigma$ error of the mean of $1.07 \%$

Session 2: $9 \mathrm{CZ3}$ standard analyses yielded a $2 \sigma$ error of the mean of $1.93 \%$

\begin{tabular}{lllllllll}
\hline 1ML2-1 & 0.070 & 171 & 279 & 1.69 & $0.09497 \pm 0.00075$ & $0.25688 \pm 0.00261$ & $1527.5 \pm 14.9$ & 96.5 \\
1ML2-8 & 0.232 & 262 & 517 & 2.04 & $0.09504 \pm 0.00078$ & $0.25790 \pm 0.00252$ & $1528.9 \pm 15.5$ & 96.7 \\
1ML2-5 & 3.913 & 326 & 572 & 1.81 & $0.09224 \pm 0.00632$ & $0.25073 \pm 0.00259$ & $1473.5 \pm 129.9$ & 97.9 \\
1ML2-10 & 4.250 & 256 & 445 & 1.80 & $0.09288 \pm 0.00432$ & $0.23290 \pm 0.00240$ & $1486.6 \pm 88.0$ & 90.8 \\
1ML2-11 & 3.107 & 284 & 472 & 1.72 & $0.09402 \pm 0.00362$ & $0.21551 \pm 0.00211$ & $1509.3 \pm 72.7$ & 83.4 \\
1ML2-12 & 0.777 & 415 & 687 & 1.71 & $0.09322 \pm 0.00157$ & $0.24262 \pm 0.00223$ & $1492.5 \pm 31.8$ & 93.8 \\
1ML2-13 & 1.260 & 195 & 322 & 1.70 & $0.09205 \pm 0.00175$ & $0.23712 \pm 0.00240$ & $1468.9 \pm 36.0$ & 93.4 \\
2ML2-21 & 0.037 & 265 & 498 & 1.94 & $0.09616 \pm 0.00069$ & $0.26961 \pm 0.00638$ & $1550.8 \pm 13.4$ & 99.2 \\
2ML2-22 & 4.673 & 283 & 461 & 1.68 & $0.10711 \pm 0.00822$ & $0.21757 \pm 0.00524$ & $1749.9 \pm 140.6$ & 72.5 \\
2ML2-23 & 0.725 & 319 & 407 & 1.32 & $0.09641 \pm 0.00160$ & $0.24507 \pm 0.00579$ & $1555.9 \pm 31.2$ & 90.8 \\
2ML2-24 & 0.392 & 348 & 568 & 1.69 & $0.09517 \pm 0.00095$ & $0.25925 \pm 0.00610$ & $1531.5 \pm 18.8$ & 97.0 \\
\hline
\end{tabular}

Sample LW10 : Musalango Gneiss (11.1352 S - 32.3463 E)

Analyses conducted during two sessions

Session $1: 12$ CZ3 standard analyses yielded a $2 \sigma$ error of the mean of $0.65 \%$

Session $2: 9 \mathrm{CZ} 3$ standard analyses yielded a $2 \sigma$ error of the mean of $1.93 \%$

\begin{tabular}{lcccccccr}
\hline 1LW10-1 & 0.000 & 55 & 47 & 0.88 & $0.10202 \pm 0.00121$ & $0.27148 \pm 0.00388$ & $1661.2 \pm 22.0$ & 93.2 \\
1LW10-2 & 0.090 & 69 & 57 & 0.85 & $0.09954 \pm 0.00117$ & $0.27964 \pm 0.00374$ & $1615.4 \pm 22.0$ & 98.4 \\
1LW10-3 & 0.150 & 75 & 63 & 0.87 & $0.09906 \pm 0.00134$ & $0.27641 \pm 0.00360$ & $1606.4 \pm 25.2$ & 97.9 \\
1LW10-4 & 0.426 & 50 & 36 & 0.75 & $0.09350 \pm 0.00189$ & $0.27859 \pm 0.00412$ & $1498.0 \pm 38.3$ & 105.8 \\
1LW10-5 & 0.280 & 64 & 42 & 0.67 & $0.09479 \pm 0.00177$ & $0.25952 \pm 0.00354$ & $1523.9 \pm 35.1$ & 97.6 \\
1LW10-6 & 0.212 & 115 & 85 & 0.76 & $0.09707 \pm 0.00115$ & $0.27708 \pm 0.00327$ & $1568.6 \pm 22.2$ & 100.5 \\
1LW10-7 & 1.840 & 84 & 55 & 0.67 & $0.08926 \pm 0.00295$ & $0.23250 \pm 0.00303$ & $1409.8 \pm 63.3$ & 95.6 \\
1LW10-9 & 0.034 & 64 & 55 & 0.89 & $0.09879 \pm 0.00115$ & $0.27653 \pm 0.00465$ & $1601.4 \pm 21.7$ & 98.3 \\
1LW10-10 & 1.601 & 59 & 45 & 0.79 & $0.09288 \pm 0.00312$ & $0.27515 \pm 0.00397$ & $1485.4 \pm 63.6$ & 105.5 \\
2LW10-11 & 0.132 & 164 & 96 & 0.60 & $0.09807 \pm 0.00129$ & $0.27897 \pm 0.00670$ & $1587.7 \pm 24.5$ & 99.9 \\
2LW10-12 & 0.205 & 48 & 35 & 0.76 & $0.10017 \pm 0.00194$ & $0.27610 \pm 0.00719$ & $1627.3 \pm 36.0$ & 96.6 \\
2LW10-13 & 0.335 & 82 & 83 & 1.05 & $0.09742 \pm 0.00192$ & $0.26469 \pm 0.00658$ & $1575.3 \pm 36.9$ & 96.1 \\
2LW10-14 & 0.176 & 93 & 83 & 0.93 & $0.10079 \pm 0.00283$ & $0.27730 \pm 0.00687$ & $1638.7 \pm 52.0$ & 96.3 \\
\hline Sample KK1 : Porphyritic granite (14.2854 S - 28.5491 E) & & & & \\
Analyses conducted during a single session. 10 CZ3 standard analyses yielded a 20 error of the mean of $0.36 \%$ & $98.5 \pm 35.3$ & 99.9 \\
\hline KK1-1 & 0.271 & 182 & 208 & 1.18 & $0.07202 \pm 0.00125$ & $0.16528 \pm 0.00111$ & 986.5 \\
KK1-2 & 0.268 & 117 & 130 & 1.15 & $0.07107 \pm 0.00163$ & $0.16747 \pm 0.00138$ & $959.3 \pm 46.9$ & 104.0 \\
KK1-3 & 0.426 & 262 & 126 & 0.50 & $0.07244 \pm 0.00233$ & $0.16346 \pm 0.00098$ & $998.3 \pm 65.3$ & 97.8 \\
KK1-4 & 0.920 & 56 & 74 & 1.35 & $0.07090 \pm 0.00391$ & $0.16310 \pm 0.00207$ & $954.6 \pm 112.6$ & 102.0 \\
KK1-5 & 0.000 & 183 & 166 & 0.94 & $0.07316 \pm 0.00073$ & $0.17005 \pm 0.00112$ & $1018.3 \pm 20.3$ & 99.4 \\
KK1-6 & 0.000 & 171 & 189 & 1.15 & $0.07442 \pm 0.00076$ & $0.16554 \pm 0.00112$ & $1052.8 \pm 20.7$ & 93.8 \\
\hline
\end{tabular}

Sample MH4 : Porphyritic granite (14.4302 S - 29.1847 E)

Analyses conducted during two sessions

Session $1: 10 \mathrm{CZ3}$ standard analyses yielded a $2 \sigma$ error of the mean of $0.98 \%$

Session 2: $10 \mathrm{CZ3}$ standard analyses yielded a $2 \sigma$ error of the mean of $0.36 \%$

\begin{tabular}{lccccccrr}
\hline MH4-1 & 0.089 & 88 & 77 & 0.90 & $0.07264 \pm 0.00091$ & $1.71845 \pm 0.03341$ & $1003.9 \pm 25.4$ & 101.7 \\
1MH4-2 & 2.762 & 167 & 124 & 0.76 & $0.07159 \pm 0.00290$ & $1.00004 \pm 0.04290$ & $974.4 \pm 82.5$ & 63.8 \\
1MH4-3 & 0.142 & 394 & 636 & 1.67 & $0.07331 \pm 0.00091$ & $1.72059 \pm 0.04192$ & $1022.6 \pm 25.1$ & 99.1 \\
2MH4-4 & 0.743 & 1418 & 444 & 0.32 & $0.07233 \pm 0.00160$ & $1.42415 \pm 0.03171$ & $995.4 \pm 44.9$ & 86.4 \\
2MH4-5 & 1.938 & 425 & 206 & 0.50 & $0.07493 \pm 0.00734$ & $1.54638 \pm 0.15199$ & $1066.7 \pm 197.0$ & 84.3 \\
2MH4-6 & 0.127 & 301 & 168 & 0.58 & $0.07465 \pm 0.00073$ & $1.61686 \pm 0.01781$ & $1059.0 \pm 19.7$ & 88.8 \\
\hline
\end{tabular}

Sample MH9 : Porphyritic granite (14.1685 S - 28.3297 E)

Analyses conducted during two sessions

Session $1: 10 \mathrm{CZ3}$ standard analyses yielded a $2 \sigma$ error of the mean of $0.98 \%$

Session $2: 10 \mathrm{CZ3}$ standard analyses yielded a $2 \sigma$ error of the mean of $0.36 \%$

\begin{tabular}{lrlllllrr} 
1MH9-1 & 0.133 & 363 & 294 & 0.84 & $0.07346 \pm 0.00065$ & $1.67234 \pm 0.02688$ & $986.2 \pm 31.0$ & 99.9 \\
1MH9-2 & 0.253 & 120 & 79 & 0.68 & $0.07302 \pm 0.00183$ & $1.69526 \pm 0.04895$ & $1050.4 \pm 30.6$ & 95.5 \\
1MH9-3 & 0.019 & 615 & 452 & 0.76 & $0.07418 \pm 0.00032$ & $1.79513 \pm 0.02427$ & $1077.0 \pm 14.6$ & 96.8 \\
2MH9-4 & 1.354 & 696 & 504 & 0.75 & $0.07486 \pm 0.00656$ & $1.75921 \pm 0.15444$ & $1064.7 \pm 176.1$ & 95.3 \\
2MH9-5 & 0.041 & 491 & 343 & 0.72 & $0.07386 \pm 0.00048$ & $1.71359 \pm 0.01305$ & $1037.8 \pm 13.1$ & 96.6 \\
2MH9-6 & 0.037 & 808 & 632 & 0.81 & $0.07342 \pm 0.00037$ & $1.76861 \pm 0.01042$ & $1025.6 \pm 10.1$ & 101.2 \\
2MH9-7 & 0.125 & 628 & 442 & 0.73 & $0.07333 \pm 0.00059$ & $1.77452 \pm 0.01590$ & $1023.1 \pm 16.2$ & 101.9 \\
2MH9-1 & 0.133 & 363 & 294 & 0.84 & $0.07346 \pm 0.00065$ & $1.67234 \pm 0.02688$ & $986.2 \pm 31.0$ & 99.9 \\
\hline
\end{tabular}


TABLE 2

(continued)

\begin{tabular}{|c|c|c|c|c|c|c|c|c|}
\hline Spot Name & $\begin{array}{l}f_{206} \\
(\%)\end{array}$ & $\begin{array}{c}\mathrm{U} \\
(\mathrm{ppm})\end{array}$ & $\begin{array}{c}\text { Th } \\
\text { (ppm) }\end{array}$ & $\mathbf{T h} / \mathbf{U}$ & $\begin{array}{c}{ }^{{ }^{207} \mathrm{~Pb}^{/ 206} \mathrm{~Pb}} \\
( \pm 1 \sigma)\end{array}$ & $\begin{array}{c}{ }^{206} \mathrm{~Pb}^{1 / 238} \mathrm{U} \\
( \pm 1 \sigma)\end{array}$ & $\begin{array}{c}{ }^{207} \mathrm{~Pb}^{1206} \mathrm{~Pb} \text { Age } \\
( \pm 1 \sigma \mathrm{Ma})\end{array}$ & $\mathrm{C}(\%)$ \\
\hline \multirow{2}{*}{\multicolumn{9}{|c|}{$\begin{array}{l}\text { Sample CC5 : Porphyritic granite }(14.2762 \mathbf{S}-\mathbf{2 8 . 5 8 5 8} \mathbf{E}) \\
\text { Analyses conducted during a single session. } 16 \mathrm{CZ} 3 \text { standard analyses yielded a } 2 \sigma \text { error of the mean of } 2.10 \%\end{array}$}} \\
\hline & & & & & & & & \\
\hline$\overline{\mathrm{CC} 5-1}$ & 0.098 & 327 & 151 & 0.48 & $0.07301 \pm 0.00078$ & $0.17914 \pm 0.00706$ & $1014.2 \pm 21.7$ & 104.7 \\
\hline CC5-2 & 0.000 & 145 & 57 & 0.40 & $0.07454 \pm 0.00109$ & $0.17549 \pm 0.00697$ & $1056.0 \pm 29.4$ & 98.7 \\
\hline CC5-3.1 & 0.000 & 195 & 102 & 0.54 & $0.07671 \pm 0.00110$ & $0.17598 \pm 0.00699$ & $1113.6 \pm 28.5$ & 93.8 \\
\hline $\mathrm{CC} 5-3.2$ & 0.684 & 338 & 204 & 0.62 & $0.07234 \pm 0.00186$ & $0.16622 \pm 0.00660$ & $995.5 \pm 52.3$ & 99.6 \\
\hline CC5-4 & 0.000 & 311 & 133 & 0.44 & $0.07398 \pm 0.00071$ & $0.17326 \pm 0.00685$ & $1040.9 \pm 19.3$ & 99.0 \\
\hline CC5-5 & 0.000 & 198 & 110 & 0.57 & $0.07375 \pm 0.00071$ & $0.17047 \pm 0.00674$ & $1034.7 \pm 19.5$ & 98.1 \\
\hline \multicolumn{9}{|c|}{ 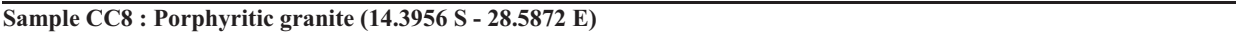 } \\
\hline \multicolumn{9}{|c|}{ Analyses conducted during a single session. $16 \mathrm{CZ} 3$ standard analyses yielded a $2 \sigma$ error of the mean of $2.10 \%$} \\
\hline CC8-1 & 0.000 & 476 & 214 & 0.46 & $0.07414 \pm 0.00053$ & $0.17374 \pm 0.00683$ & $1045.3 \pm 14.4$ & 98.8 \\
\hline CC8-2 & 0.000 & 257 & 139 & 0.56 & $0.07360 \pm 0.00062$ & $0.16806 \pm 0.00664$ & $1030.4 \pm 17.0$ & 97.2 \\
\hline CC8-3 & 0.000 & 393 & 178 & 0.47 & $0.07376 \pm 0.00050$ & $0.17462 \pm 0.00687$ & $1034.8 \pm 13.8$ & 100.3 \\
\hline CC $8-4$ & 0.000 & 406 & 260 & 0.66 & $0.07421 \pm 0.00049$ & $0.17340 \pm 0.00682$ & $1047.2 \pm 13.2$ & 98.4 \\
\hline CC8-5 & 0.652 & 361 & 164 & 0.47 & $0.07191 \pm 0.00146$ & $0.15855 \pm 0.00625$ & $983.3 \pm 41.3$ & 96.5 \\
\hline CC8-6 & 0.063 & 570 & 230 & 0.42 & $0.07341 \pm 0.00048$ & $0.17011 \pm 0.00668$ & $1025.4 \pm 13.3$ & 98.8 \\
\hline
\end{tabular}

Sample MK7 : Porphyritic granite (13.9592 S - 29.1176 E)

Analyses conducted during two sessions

Session 1 : $10 \mathrm{CZ3}$ standard analyses yielded a $2 \sigma$ error of the mean of $0.98 \%$

Session $2: 10 \mathrm{CZ} 3$ standard analyses yielded a $2 \sigma$ error of the mean of $0.36 \%$

\begin{tabular}{|c|c|c|c|c|c|c|c|c|}
\hline$\overline{1 \mathrm{MK} 7-1}$ & 0.660 & 199 & 415 & 2.16 & $0.07376 \pm 0.00118$ & $1.65756 \pm 0.03473$ & $1034.9 \pm 32.2$ & 94.1 \\
\hline $1 \mathrm{MK} 7-2 \mathrm{c}$ & 1.670 & 333 & 284 & 0.88 & $0.12323 \pm 0.00248$ & $4.45448 \pm 0.10767$ & $2003.4 \pm 35.8$ & 74.9 \\
\hline $1 \mathrm{MK} 7-2 \mathrm{r}$ & 0.530 & 1815 & 64 & 0.04 & $0.06881 \pm 0.00056$ & $1.39160 \pm 0.02089$ & $892.9 \pm 16.7$ & 98.8 \\
\hline $2 \mathrm{MK} 7-3$ & 1.569 & 261 & 422 & 1.67 & $0.07879 \pm 0.00201$ & $1.59315 \pm 0.04186$ & $1166.9 \pm 50.6$ & 75.6 \\
\hline 2MK7-4 & 1.296 & 192 & 228 & 1.23 & $0.07985 \pm 0.00253$ & $1.28480 \pm 0.04190$ & $1193.2 \pm 62.6$ & 59.6 \\
\hline 2MK7-5 & 2.088 & 1199 & 175 & 0.15 & $0.06873 \pm 0.00366$ & $1.13515 \pm 0.06128$ & $890.7 \pm 110.0$ & 81.9 \\
\hline \multicolumn{9}{|c|}{ Sample ND1 : Porphyritic granite (13.4914 S - 29.8885 E) } \\
\hline$\overline{\text { ND1-1 }}$ & 1.348 & 1300 & 486 & 0.39 & $0.07944 \pm 0.00934$ & $0.16120 \pm 0.00216$ & $1183.1 \pm 232.3$ & 81.4 \\
\hline ND1-2 & 0.000 & 288 & 419 & 1.50 & $0.07371 \pm 0.00052$ & $0.17831 \pm 0.00219$ & $1033.5 \pm 14.3$ & 102.3 \\
\hline ND1-3 & 0.777 & 238 & 266 & 1.16 & $0.08212 \pm 0.00285$ & $0.16971 \pm 0.00211$ & $1248.3 \pm 67.9$ & 81.0 \\
\hline ND1-4 & 0.291 & 1057 & 481 & 0.47 & $0.07402 \pm 0.00165$ & $0.17261 \pm 0.00190$ & $1042.0 \pm 44.9$ & 98.5 \\
\hline ND1-5r & 0.512 & 999 & 532 & 0.55 & $0.07818 \pm 0.00160$ & $0.16041 \pm 0.00176$ & $1151.5 \pm 40.6$ & 83.3 \\
\hline ND1-5c & 0.003 & 1864 & 493 & 0.27 & $0.07314 \pm 0.00025$ & $0.17789 \pm 0.00201$ & $1017.7 \pm 6.9$ & 103.7 \\
\hline ND1-7 & 4.316 & 1926 & 917 & 0.49 & $0.10095 \pm 0.02577$ & $0.15316 \pm 0.00437$ & $1641.7 \pm 473.8$ & 56.0 \\
\hline ND1-8 & 6.367 & 1718 & 490 & 0.29 & $0.12801 \pm 0.08145$ & $0.14736 \pm 0.00752$ & $2070.8 \pm 1121.2$ & 42.8 \\
\hline ND1-9 & 0.000 & 1977 & 55 & 0.03 & $0.07348 \pm 0.00021$ & $0.17610 \pm 0.00203$ & $1027.2 \pm 5.8$ & 101.8 \\
\hline ND1-10 & 0.014 & 2459 & 1017 & 0.43 & $0.07322 \pm 0.00024$ & $0.17671 \pm 0.00191$ & $1020.2 \pm 6.6$ & 102.8 \\
\hline
\end{tabular}

Sample ND4 : Granodiorite (13.3736 S - 29.9484 E)

Analyses conducted during a single session. $14 \mathrm{CZ3}$ standard analyses yielded a $2 \sigma$ error of the mean of $0.66 \%$

\begin{tabular}{llllllllr}
\hline ND4-1 & 0.000 & 324 & 370 & 1.18 & $0.07357 \pm 0.00053$ & $0.17253 \pm 0.00205$ & $1029.7 \pm 14.7$ & 99.6 \\
ND4-2 & 0.071 & 271 & 288 & 1.10 & $0.07353 \pm 0.00078$ & $0.17504 \pm 0.00206$ & $1028.7 \pm 21.6$ & 101.1 \\
ND4-3 & 0.071 & 398 & 650 & 1.69 & $0.07364 \pm 0.00053$ & $0.17264 \pm 0.00200$ & $1031.7 \pm 14.5$ & 99.5 \\
ND4-4 & 0.011 & 306 & 328 & 1.11 & $0.07444 \pm 0.00054$ & $0.17156 \pm 0.00210$ & $1053.5 \pm 14.5$ & 96.9 \\
\hline
\end{tabular}

Sample ND5 : Syeno-granite (13.2011 S - 29.9472 E)

Analyses conducted during a single session. $14 \mathrm{CZ} 3$ standard analyses yielded a $2 \sigma$ error of the mean of $0.66 \%$

\begin{tabular}{lrllllllr}
\hline ND5-1 & 0.721 & 263 & 332 & 1.31 & $0.07687 \pm 0.00134$ & $0.16182 \pm 0.00190$ & $1117.9 \pm 34.8$ & 86.5 \\
ND5-2 & 0.097 & 230 & 311 & 1.39 & $0.07293 \pm 0.00090$ & $0.17429 \pm 0.00208$ & $1012.1 \pm 24.9$ & 102.3 \\
ND5-3 & 0.036 & 241 & 253 & 1.09 & $0.07347 \pm 0.00083$ & $0.17202 \pm 0.00204$ & $1027.0 \pm 23.0$ & 99.6 \\
ND5-4 & 0.623 & 242 & 401 & 1.72 & $0.07501 \pm 0.00128$ & $0.16700 \pm 0.00204$ & $1068.7 \pm 34.4$ & 93.2 \\
\hline
\end{tabular}

Sample FW1 : Porphyritic granite (13.6684 S - 29.6242 E)

Analyses conducted during a single session. $7 \mathrm{CZ} 3$ standard analyses yielded a $2 \sigma$ error of the mean of $1.65 \%$

\begin{tabular}{lccccccrr} 
Analyses conducted during a single session. 7 CZ3 standard analyses yielded a $2 \sigma$ error of the mean of $1.65 \%$ \\
\hline FW1-2 & 0.452 & 2020 & 422 & 0.22 & $0.07069 \pm 0.00064$ & $1.69632 \pm 0.03287$ & $948.5 \pm 18.5$ \\
FW1-3 & 0.108 & 1069 & 382 & 0.37 & $0.07417 \pm 0.00057$ & $1.78742 \pm 0.03402$ & $1046.0 \pm 15.6$ \\
FW1-4 & 0.484 & 203 & 359 & 1.82 & $0.07207 \pm 0.00183$ & $1.77925 \pm 0.05690$ & $988.0 \pm 51.6$ & 107.5 \\
FW1-5 & 1.112 & 389 & 499 & 1.32 & $0.07156 \pm 0.00266$ & $1.64344 \pm 0.06824$ & $973.4 \pm 75.9$ \\
FW1-6 & 0.000 & 1327 & 148 & 0.12 & $0.07464 \pm 0.00043$ & $1.88767 \pm 0.03439$ & $1058.9 \pm 11.6$ & 102.0 \\
FW1-7 & 1.112 & 712 & 776 & 1.13 & $0.07299 \pm 0.00166$ & $1.70267 \pm 0.04924$ & $1013.7 \pm 46.2$ \\
\hline
\end{tabular}


TABLE 2

(continued)

\begin{tabular}{|c|c|c|c|c|c|c|c|c|}
\hline Spot Name & $\begin{array}{c}f_{206} \\
(\%) \\
\end{array}$ & $\begin{array}{c}\mathrm{U} \\
(\mathrm{ppm})\end{array}$ & $\begin{array}{c}\text { Th } \\
(\mathbf{p p m})\end{array}$ & $\mathbf{T h} / \mathbf{U}$ & $\begin{array}{c}{ }^{{ }^{207} \mathbf{P b}^{/ 206} \mathbf{P b}} \\
( \pm 1 \sigma) \\
\end{array}$ & $\begin{array}{c}{ }^{206} \mathbf{P b}^{/ 238} \mathrm{U} \\
( \pm 1 \sigma) \\
\end{array}$ & $\begin{array}{c}{ }^{{ }^{207} \mathrm{~Pb}^{/ 206} \mathrm{~Pb} \text { Age }} \\
( \pm 1 \sigma \mathrm{Ma}) \\
\end{array}$ & $\mathrm{C}(\%)$ \\
\hline \multicolumn{9}{|c|}{ Sample FW2 : Aplite in porphyritic granite (13.6684 S - 29.6242 E) } \\
\hline \multicolumn{9}{|c|}{ Analyses conducted during a single session. 7 CZ3 standard analyses yielded a $2 \sigma$ error of the mean of $1.65 \%$} \\
\hline FW2-1 & 0.391 & 120 & 70 & 0.60 & $0.12523 \pm 0.00195$ & $6.35520 \pm 0.16284$ & $2032.1 \pm 27.5$ & 99.4 \\
\hline FW2-2 & 1.017 & 345 & 94 & 0.28 & $0.08254 \pm 0.00436$ & $1.99952 \pm 0.11282$ & $1258.3 \pm 103.2$ & 82.9 \\
\hline FW2-3 & 0.089 & 968 & 96 & 0.10 & $0.12607 \pm 0.00055$ & $6.44670 \pm 0.11521$ & $2043.9 \pm 7.8$ & 99.5 \\
\hline FW2-4 & 0.210 & 191 & 87 & 0.47 & $0.19366 \pm 0.00273$ & $14.32643 \pm 0.33874$ & $2773.5 \pm 23.2$ & 99.8 \\
\hline FW2-5 & 0.325 & 209 & 72 & 0.36 & $0.11233 \pm 0.00173$ & $4.56871 \pm 0.11198$ & $1837.4 \pm 27.9$ & 90.7 \\
\hline FW2-6 & 0.056 & 1375 & 122 & 0.09 & $0.12414 \pm 0.00067$ & $6.47916 \pm 0.11706$ & $2016.5 \pm 9.5$ & 102.6 \\
\hline FW2-7 & 0.134 & 307 & 22 & 0.07 & $0.12370 \pm 0.00145$ & $6.33304 \pm 0.13892$ & $2010.3 \pm 20.8$ & 101.3 \\
\hline FW2-8 & 0.597 & 77 & 40 & 0.54 & $0.12984 \pm 0.00281$ & $7.04491 \pm 0.22442$ & $2095.8 \pm 38.1$ & 102.1 \\
\hline FW2-9 & 0.465 & 114 & 237 & 2.16 & $0.13000 \pm 0.00483$ & $6.53413 \pm 0.31931$ & $2097.9 \pm 65.2$ & 95.5 \\
\hline FW2-10 & 0.884 & 185 & 87 & 0.49 & $0.12534 \pm 0.00216$ & $6.23497 \pm 0.16391$ & $2033.6 \pm 30.5$ & 97.7 \\
\hline FW2-11 & 0.086 & 343 & 221 & 0.67 & $0.11927 \pm 0.00262$ & $6.15944 \pm 0.17685$ & $1945.3 \pm 39.3$ & 105.4 \\
\hline FW2-12 & 0.549 & 157 & 115 & 0.76 & $0.10069 \pm 0.00251$ & $4.11285 \pm 0.13133$ & $1636.9 \pm 46.4$ & 102.2 \\
\hline FW2-13 & 0.066 & 648 & 83 & 0.13 & $0.16268 \pm 0.00073$ & $8.84789 \pm 0.16048$ & $2483.7 \pm 7.6$ & 86.3 \\
\hline
\end{tabular}

Sample SASA2 : Sasa granite (13.0040 S - 30.0832 E)

Analyses conducted during a single session. $16 \mathrm{CZ} 3$ standard analyses yielded a $2 \sigma$ error of the mean of $1.18 \%$

\begin{tabular}{|c|c|c|c|c|c|c|c|c|}
\hline$\overline{\text { SASA2-1 }}$ & 2.481 & 48 & 110 & 2.38 & $0.06921 \pm 0.01622$ & $0.17652 \pm 0.00516$ & $905.1 \pm 482.9$ & 115.8 \\
\hline SASA2-2 & 0.707 & 912 & 780 & 0.88 & $0.07271 \pm 0.00054$ & $0.17402 \pm 0.00413$ & $1005.8 \pm 15.1$ & 102.8 \\
\hline SASA2-3c & 0.268 & 465 & 281 & 0.62 & $0.07472 \pm 0.00128$ & $0.16978 \pm 0.00398$ & $1060.9 \pm 34.5$ & 95.3 \\
\hline SASA2-3r & 0.823 & 1932 & 467 & 0.25 & $0.07254 \pm 0.00113$ & $0.15492 \pm 0.00333$ & $1001.0 \pm 31.6$ & 92.8 \\
\hline SASA2-5 & 0.301 & 417 & 404 & 1.00 & $0.07653 \pm 0.00149$ & $0.14188 \pm 0.00340$ & $1109.0 \pm 39.0$ & 77.1 \\
\hline SASA2-10 & 1.723 & 228 & 279 & 1.26 & $0.07278 \pm 0.00333$ & $0.16983 \pm 0.00427$ & $1008.0 \pm 92.7$ & 100.3 \\
\hline SASA2-11 & 0.294 & 1974 & 66 & 0.03 & $0.07403 \pm 0.00064$ & $0.16416 \pm 0.00387$ & $1042.3 \pm 17.3$ & 94.0 \\
\hline SASA2-12c & 0.858 & 1059 & 371 & 0.36 & $0.07295 \pm 0.00087$ & $0.17077 \pm 0.00367$ & $1012.7 \pm 24.1$ & 100.4 \\
\hline SASA2-12r & 0.311 & 1133 & 326 & 0.30 & $0.07414 \pm 0.00265$ & $0.17338 \pm 0.00375$ & $1045.3 \pm 72.1$ & 98.6 \\
\hline SASA2-15 & 0.890 & 186 & 266 & 1.48 & $0.07038 \pm 0.00357$ & $0.17803 \pm 0.00399$ & $939.5 \pm 104.0$ & 112.4 \\
\hline SASA2-17 & 0.945 & 252 & 329 & 1.35 & $0.07226 \pm 0.00112$ & $0.16976 \pm 0.00407$ & $993.3 \pm 31.5$ & 101.8 \\
\hline SASA2-18 & 1.335 & 193 & 279 & 1.49 & $0.07682 \pm 0.00499$ & $0.14176 \pm 0.00329$ & $1116.6 \pm 129.6$ & 76.5 \\
\hline \multicolumn{9}{|c|}{ Sample SER53 : Porphyritic granite (13.1270 S - 30.1349 E) } \\
\hline SER53-1 & 0.041 & 257 & 248 & 1.00 & $0.07388 \pm 0.00076$ & $0.17373 \pm 0.00329$ & $1038.2 \pm 20.8$ & 99.5 \\
\hline SER53-2 & 0.001 & 252 & 123 & 0.50 & $0.07371 \pm 0.00075$ & $0.17480 \pm 0.00336$ & $1033.7 \pm 20.4$ & 100.5 \\
\hline SER53-3 & 0.416 & 117 & 198 & 1.74 & $0.07069 \pm 0.00229$ & $0.16569 \pm 0.00341$ & $948.5 \pm 66.4$ & 104.2 \\
\hline SER53-4 & 0.000 & 813 & 761 & 0.97 & $0.07387 \pm 0.00042$ & $0.17557 \pm 0.00321$ & $1037.9 \pm 11.5$ & 100.5 \\
\hline SER53-5 & 0.000 & 317 & 292 & 0.95 & $0.07488 \pm 0.00069$ & $0.17544 \pm 0.00332$ & $1065.4 \pm 18.5$ & 97.8 \\
\hline SER53-6 & 0.054 & 244 & 261 & 1.10 & $0.07259 \pm 0.00080$ & $0.17680 \pm 0.00341$ & $1002.5 \pm 22.5$ & 104.7 \\
\hline SER53-7 & 0.101 & 513 & 455 & 0.92 & $0.07327 \pm 0.00073$ & $0.16920 \pm 0.00344$ & $1021.4 \pm 20.1$ & 98.7 \\
\hline SER53-8 & 0.170 & 457 & 409 & 0.92 & $0.07259 \pm 0.00068$ & $0.17488 \pm 0.00331$ & $1002.6 \pm 19.0$ & 103.6 \\
\hline SER53-9 & 0.420 & 189 & 197 & 1.08 & $0.07034 \pm 0.00173$ & $0.16331 \pm 0.00334$ & $938.2 \pm 50.6$ & 103.9 \\
\hline SER53-10 & 4.930 & 346 & 392 & 1.17 & $0.07239 \pm 0.00472$ & $0.16601 \pm 0.00330$ & $997.1 \pm 132.5$ & 99.3 \\
\hline
\end{tabular}

Sample SER64 : Porphyritic granite (13.3873 S - 30.3958 E)

Analyses conducted during a single session. $14 \mathrm{CZ3}$ standard analyses yielded a $2 \sigma$ error of the mean of $1.11 \%$

\begin{tabular}{|c|c|c|c|c|c|c|c|c|}
\hline SER64-1 & 0.632 & 171 & 382 & 2.31 & $0.07237 \pm 0.00174$ & $0.14368 \pm 0.00283$ & $996.3 \pm 48.8$ & 86.9 \\
\hline SER64-2 & 0.000 & 260 & 71 & 0.28 & $0.07517 \pm 0.00078$ & $0.17481 \pm 0.00332$ & $1073.1 \pm 20.9$ & 96.8 \\
\hline SER64-3 & 0.000 & 1252 & 248 & 0.20 & $0.07398 \pm 0.00036$ & $0.18155 \pm 0.00328$ & $1040.8 \pm 9.9$ & 103.3 \\
\hline SER64-4 & 0.000 & 237 & 712 & 3.11 & $0.07339 \pm 0.00078$ & $0.17228 \pm 0.00333$ & $1024.7 \pm 21.6$ & 100.0 \\
\hline SER64-5 & 0.000 & 449 & 670 & 1.54 & $0.07283 \pm 0.00066$ & $0.17375 \pm 0.00328$ & $1009.1 \pm 18.3$ & 102.3 \\
\hline SER64-6 & 0.701 & 50 & 99 & 2.04 & $0.06717 \pm 0.00406$ & $0.16809 \pm 0.00428$ & $842.9 \pm 125.7$ & 118.8 \\
\hline SER64-7 & 0.000 & 1038 & 140 & 0.14 & $0.07344 \pm 0.00042$ & $0.17694 \pm 0.00349$ & $1026.0 \pm 11.6$ & 102.4 \\
\hline SER64-8 & 0.603 & 898 & 292 & 0.34 & $0.07516 \pm 0.00099$ & $0.15197 \pm 0.00281$ & $1072.8 \pm 26.5$ & 85.0 \\
\hline SER64-9 & 0.000 & 889 & 979 & 1.14 & $0.12301 \pm 0.00044$ & $0.37536 \pm 0.00694$ & $2000.4 \pm 6.4$ & 102.7 \\
\hline SER64-10 & 0.292 & 213 & 562 & 2.73 & $0.07507 \pm 0.00403$ & $0.17522 \pm 0.00373$ & $1070.4 \pm 107.8$ & 97.2 \\
\hline \multicolumn{9}{|c|}{ Sample SQG2 : Serenje Quarry Granite (13.2148 S - 30.2486 E) } \\
\hline SQG2-72 & 0.000 & 460 & 463 & 1.04 & $0.07474 \pm 0.00051$ & $0.17773 \pm 0.00270$ & $1061.6 \pm 13.7$ & 99.3 \\
\hline SQG2-70 & 0.000 & 187 & 88 & 0.49 & $0.07616 \pm 0.00114$ & $0.18443 \pm 0.00825$ & $1099.4 \pm 29.9$ & 99.3 \\
\hline SQG2-26 & 0.004 & 402 & 187 & 0.48 & $0.07480 \pm 0.00040$ & $0.16799 \pm 0.00253$ & $1063.0 \pm 10.8$ & 94.2 \\
\hline SQG2-60 & 0.012 & 689 & 348 & 0.52 & $0.07370 \pm 0.00031$ & $0.16831 \pm 0.00249$ & $1033.3 \pm 8.5$ & 97.1 \\
\hline SQG2-7 & 0.027 & 699 & 259 & 0.38 & $0.07329 \pm 0.00034$ & $0.17155 \pm 0.00253$ & $1021.9 \pm 9.5$ & 99.9 \\
\hline SQG2-17 & 0.040 & 136 & 90 & 0.68 & $0.07244 \pm 0.00083$ & $0.17304 \pm 0.00273$ & $998.4 \pm 23.3$ & 103.0 \\
\hline SQG2-77 & 0.042 & 133 & 97 & 0.76 & $0.07241 \pm 0.00069$ & $0.18131 \pm 0.00288$ & $997.6 \pm 19.3$ & 107.7 \\
\hline SQG2-48 & 0.043 & 702 & 346 & 0.51 & $0.07362 \pm 0.00034$ & $0.16623 \pm 0.00245$ & $1031.2 \pm 9.4$ & 96.1 \\
\hline SQG2-20 & 0.074 & 411 & 249 & 0.62 & $0.07357 \pm 0.00061$ & $0.17071 \pm 0.00257$ & $1029.8 \pm 16.9$ & 98.7 \\
\hline SQG2-1 & 0.088 & 233 & 144 & 0.64 & $0.07264 \pm 0.00060$ & $0.17245 \pm 0.00263$ & $1003.8 \pm 16.8$ & 102.2 \\
\hline SQG2-14 & 1.201 & 575 & 284 & 0.51 & $0.07218 \pm 0.00129$ & $0.16418 \pm 0.00251$ & $990.9 \pm 36.3$ & 98.9 \\
\hline
\end{tabular}


TABLE 2

(continued)

\begin{tabular}{|c|c|c|c|c|c|c|c|c|}
\hline Spot Name & $\begin{array}{l}f_{206} \\
(\%)\end{array}$ & $\begin{array}{c}\mathrm{U} \\
(\mathrm{ppm})\end{array}$ & $\begin{array}{c}\text { Th } \\
(\mathbf{p p m})\end{array}$ & $\mathbf{T h} / \mathbf{U}$ & $\begin{array}{c}{ }^{207} \mathrm{~Pb}^{1206} \mathrm{~Pb} \\
( \pm 1 \sigma)\end{array}$ & $\begin{array}{c}{ }^{206} \mathrm{~Pb}^{1238} \mathrm{U} \\
( \pm 1 \sigma)\end{array}$ & $\begin{array}{c}{ }^{207} \mathrm{~Pb}^{1206} \mathrm{~Pb} \text { Age } \\
( \pm 1 \sigma \mathrm{Ma})\end{array}$ & $\mathrm{C}(\%)$ \\
\hline \multirow{2}{*}{\multicolumn{9}{|c|}{ Sample KN2a : Porphyritic granite (13.0991 S - 30.8204 E) }} \\
\hline & & & & & & & & \\
\hline KN2a-1 & 0.000 & 204 & 282 & 1.43 & $0.07378 \pm 0.00071$ & $0.17375 \pm 0.00411$ & $1035.6 \pm 19.4$ & 99.7 \\
\hline KN2a-2 & 0.018 & 225 & 117 & 0.54 & $0.08606 \pm 0.00084$ & $0.16993 \pm 0.00347$ & $1339.6 \pm 18.8$ & 75.5 \\
\hline KN2a-3 & 0.000 & 116 & 177 & 1.58 & $0.07873 \pm 0.00095$ & $0.17796 \pm 0.00374$ & $1165.3 \pm 24.0$ & 90.6 \\
\hline KN2a-4 & 0.000 & 165 & 146 & 0.91 & $0.08220 \pm 0.00130$ & $0.17712 \pm 0.00365$ & $1250.3 \pm 30.8$ & 84.1 \\
\hline KN2a-5 & 0.000 & 1421 & 882 & 0.64 & $0.07915 \pm 0.00119$ & $0.17420 \pm 0.00352$ & $1176.0 \pm 29.7$ & 88.0 \\
\hline KN2a-6 & 0.056 & 131 & 147 & 1.16 & $0.07527 \pm 0.00096$ & $0.17795 \pm 0.00370$ & $1075.7 \pm 25.7$ & 98.1 \\
\hline KN2a-7 & 0.000 & 236 & 176 & 0.77 & $0.07668 \pm 0.00075$ & $0.17284 \pm 0.00352$ & $1113.0 \pm 19.6$ & 92.3 \\
\hline $\mathrm{KN} 2 \mathrm{a}-8$ & 0.066 & 247 & 144 & 0.60 & $0.07634 \pm 0.00082$ & $0.17257 \pm 0.00351$ & $1103.9 \pm 21.4$ & 93.0 \\
\hline
\end{tabular}

Sample KN5 : Porphyritic granite (12.9634 S - 30.8840 E)

Analyses conducted during a single session. $12 \mathrm{CZ3}$ standard analyses yielded a $2 \sigma$ error of the mean of $1.56 \%$

\begin{tabular}{lcccccccr}
\hline KN5-1 & 0.000 & 456 & 212 & 0.48 & $0.07463 \pm 0.00046$ & $0.17865 \pm 0.00358$ & $1058.6 \pm 12.5$ & 100.1 \\
KN5-2 & 0.098 & 181 & 356 & 2.03 & $0.08078 \pm 0.00128$ & $0.17909 \pm 0.00369$ & $1216.0 \pm 31.1$ & 87.3 \\
KN5-3r & 0.050 & 2299 & 231 & 0.10 & $0.07580 \pm 0.00109$ & $0.13595 \pm 0.00294$ & $1089.8 \pm 28.9$ & 75.4 \\
KN5-3c & 0.025 & 332 & 177 & 0.55 & $0.07797 \pm 0.00081$ & $0.17663 \pm 0.00356$ & $1146.1 \pm 20.7$ & 91.5 \\
KN5-4 & 0.000 & 273 & 138 & 0.52 & $0.07652 \pm 0.00075$ & $0.17761 \pm 0.00360$ & $1108.6 \pm 19.6$ & 95.1 \\
KN5-5 & 0.021 & 247 & 120 & 0.50 & $0.07979 \pm 0.00066$ & $0.17543 \pm 0.00356$ & $1191.8 \pm 16.3$ & 87.4 \\
KN5-6 & 0.000 & 338 & 180 & 0.55 & $0.07487 \pm 0.00054$ & $0.17867 \pm 0.00360$ & $1065.1 \pm 14.4$ & 99.5 \\
KN5-7 & 0.000 & 281 & 132 & 0.49 & $0.07650 \pm 0.00060$ & $0.17752 \pm 0.00359$ & $1108.1 \pm 15.6$ & 95.1 \\
KN5-8 & 0.000 & 298 & 153 & 0.53 & $0.07624 \pm 0.00059$ & $0.18036 \pm 0.00365$ & $1101.3 \pm 15.4$ & 97.1 \\
\hline
\end{tabular}

Sample KN7 : Porphyritic granite (13.1872 S - 30.9113 E)

Analyses conducted during a single session. $12 \mathrm{CZ3}$ standard analyses yielded a $2 \sigma$ error of the mean of $1.56 \%$

\begin{tabular}{lcccccccr}
\multicolumn{1}{c}{ Analyses conducted during a single session. 12 C Z 3 standard analyses yielded a $\sigma$ error of the mean of $1.56 \%$} & \\
KN7-1 & 0.003 & 973 & 825 & 0.88 & $0.12417 \pm 0.00028$ & $0.36971 \pm 0.00738$ & $2017.0 \pm 4.1$ & 100.5 \\
KN7-3r & 0.000 & 506 & 155 & 0.32 & $0.12212 \pm 0.00108$ & $0.33971 \pm 0.00680$ & $1987.4 \pm 15.8$ & 94.9 \\
KN7-3c & 0.399 & 2878 & 39994 & 14.36 & $0.19049 \pm 0.01292$ & $0.07975 \pm 0.00159$ & $2746.4 \pm 111.5$ & 18.0 \\
KN7-4 & 0.000 & 184 & 258 & 1.45 & $0.19596 \pm 0.00156$ & $0.41147 \pm 0.00899$ & $2792.8 \pm 13.1$ & 79.5 \\
KN7-5 & 0.049 & 230 & 80 & 0.15 & $0.12215 \pm 0.00064$ & $0.37122 \pm 0.00753$ & $1987.8 \pm 9.3$ & 102.4 \\
KN7-6 & 0.010 & 259 & 818 & 0.39 & $0.11920 \pm 0.00307$ & $0.34343 \pm 0.00939$ & $1944.3 \pm 46.0$ & 97.9 \\
KN7-7 & 0.032 & 740 & 235 & 0.33 & $0.07331 \pm 0.00069$ & $0.15308 \pm 0.00310$ & $1276.5 \pm 16.2$ & 71.9 \\
\hline
\end{tabular}

Sample KN8 : Porphyritic granite (13.2260 S - 30.8976 E)

Analyses conducted during a single session. $12 \mathrm{CZ} 3$ standard analyses yielded a $2 \sigma$ error of the mean of $1.56 \%$

\begin{tabular}{lcccccccc}
\hline KN8-1r & 0.018 & 245 & 191 & 0.81 & $0.08522 \pm 0.00068$ & $0.19321 \pm 0.00399$ & $1320.4 \pm 15.6$ & 86.2 \\
KN8-1c & 0.007 & 117 & 58 & 0.51 & $0.12664 \pm 0.00092$ & $0.37348 \pm 0.00773$ & $2051.8 \pm 12.8$ & 99.7 \\
KN8-2 & 0.000 & 496 & 1171 & 2.44 & $0.08071 \pm 0.00091$ & $0.16912 \pm 0.00338$ & $1214.4 \pm 22.2$ & 82.9 \\
KN8-3 & 0.000 & 107 & 124 & 1.19 & $0.08184 \pm 0.00128$ & $0.17658 \pm 0.00371$ & $1241.6 \pm 30.7$ & 84.4 \\
KN8-4c & 0.031 & 406 & 88 & 0.23 & $0.12828 \pm 0.00255$ & $0.32698 \pm 0.00657$ & $2074.5 \pm 35.0$ & 87.9 \\
KN8-4r & 0.000 & 315 & 176 & 0.58 & $0.07506 \pm 0.00059$ & $0.16945 \pm 0.00343$ & $1070.0 \pm 15.8$ & 94.3 \\
KN8-5 & 0.000 & 382 & 256 & 0.69 & $0.07682 \pm 0.00057$ & $0.16490 \pm 0.00331$ & $1116.4 \pm 14.9$ & 88.1 \\
KN8-6 & 0.033 & 310 & 228 & 0.76 & $0.07609 \pm 0.00140$ & $0.17208 \pm 0.00356$ & $1097.4 \pm 36.7$ & 93.3 \\
KN8-7 & 0.000 & 321 & 134 & 0.43 & $0.10008 \pm 0.00164$ & $0.24025 \pm 0.00483$ & $1625.6 \pm 30.4$ & 85.4 \\
KN8-8 & 0.002 & 378 & 348 & 0.95 & $0.07472 \pm 0.00063$ & $0.17771 \pm 0.00357$ & $1060.9 \pm 17.0$ & 99.4 \\
KN8-9 & 0.000 & 113 & 164 & 1.50 & $0.08671 \pm 0.00137$ & $0.17318 \pm 0.00364$ & $1354.1 \pm 30.4$ & 76.0 \\
KN8-10 & 0.002 & 725 & 586 & 0.83 & $0.07918 \pm 0.00047$ & $0.17180 \pm 0.00342$ & $1176.7 \pm 11.9$ & 86.9 \\
KN8-11 & 0.091 & 215 & 267 & 1.29 & $0.07687 \pm 0.00091$ & $0.17315 \pm 0.00353$ & $1117.9 \pm 23.6$ & 92.1 \\
\hline
\end{tabular}

Sample CHT6 : Porphyritic granite (12.7766 S - 30.9725 E)

Analyses conducted during a single session. $7 \mathrm{CZ3}$ standard analyses yielded a $2 \sigma$ error of the mean of $1.65 \%$

\begin{tabular}{lcccccccr}
\hline CHT6-1 & 0.869 & 144 & 117 & 0.84 & $0.07304 \pm 0.00306$ & $1.74172 \pm 0.08690$ & $1015.1 \pm 84.8$ & 101.3 \\
CHT6-2 & 1.150 & 147 & 178 & 1.25 & $0.06558 \pm 0.00336$ & $1.53049 \pm 0.08469$ & $792.9 \pm 107.5$ & 127.1 \\
CHT6-3 & 0.460 & 415 & 282 & 0.70 & $0.07373 \pm 0.00161$ & $1.71450 \pm 0.04876$ & $1034.2 \pm 44.1$ & 97.1 \\
CHT6-4 & 0.225 & 300 & 214 & 0.74 & $0.07371 \pm 0.00113$ & $1.74871 \pm 0.04221$ & $1033.5 \pm 30.9$ & 99.0 \\
CHT6-5 & 2.414 & 148 & 111 & 0.77 & $0.06606 \pm 0.00561$ & $1.43724 \pm 0.12602$ & $808.3 \pm 177.7$ & 116.9 \\
CHT6-6 & 0.680 & 164 & 175 & 1.11 & $0.07541 \pm 0.00311$ & $1.73153 \pm 0.07990$ & $1079.3 \pm 82.9$ & 92.0 \\
CHT6-7 & 0.778 & 113 & 99 & 0.91 & $0.07232 \pm 0.00356$ & $1.66512 \pm 0.09000$ & $994.9 \pm 100.2$ & 100.1 \\
CHT6-8 & 1.591 & 93 & 124 & 1.38 & $0.06590 \pm 0.00529$ & $1.50833 \pm 0.12608$ & $803.3 \pm 168.0$ & 123.2 \\
CHT6-9 & 1.253 & 140 & 143 & 1.06 & $0.07048 \pm 0.00587$ & $1.62685 \pm 0.13995$ & $942.3 \pm 170.7$ & 105.9 \\
\hline Sample ZM36 : Mununga Quarry Granite (12.6259 S -31.0434 E) \\
Analyses conducted during a single session. 14 CZ3 standard analyses yielded a 26 error of the mean of $1.11 \%$ & $11 \% 7.5 \pm 57.4$ & 90.8 \\
\hline ZM36-1 & 0.000 & 91 & 94 & 1.07 & $0.07842 \pm 0.00227$ & $0.17709 \pm 0.00381$ & 1157.1 \\
ZM36-2 & 0.193 & 145 & 138 & 0.98 & $0.07180 \pm 0.00144$ & $0.17875 \pm 0.00354$ & $980.3 \pm 40.7$ & 108.1 \\
ZM36-3 & 0.000 & 32 & 30 & 0.95 & $0.08094 \pm 0.00861$ & $0.17282 \pm 0.00519$ & $1220.0 \pm 209.1$ & 84.2 \\
ZM36-4 & 0.026 & 491 & 269 & 0.57 & $0.07344 \pm 0.00055$ & $0.17008 \pm 0.00315$ & $1026.2 \pm 15.1$ & 98.7 \\
ZM36-5 & 0.014 & 487 & 298 & 0.63 & $0.07348 \pm 0.00063$ & $0.17624 \pm 0.00326$ & $1027.3 \pm 17.5$ & 101.9 \\
ZM36-6 & 0.358 & 64 & 65 & 1.05 & $0.06967 \pm 0.00246$ & $0.16523 \pm 0.00395$ & $918.6 \pm 72.8$ & 107.3 \\
\hline
\end{tabular}


TABle 2

(continued)

\begin{tabular}{|c|c|c|c|c|c|c|c|c|}
\hline Spot Name & $\begin{array}{c}f_{206} \\
(\%)\end{array}$ & $\begin{array}{c}\mathrm{U} \\
(\mathrm{ppm})\end{array}$ & $\begin{array}{c}\text { Th } \\
(\mathrm{ppm})\end{array}$ & $\mathbf{T h} / \mathbf{U}$ & $\begin{array}{c}{ }^{207} \mathrm{~Pb}^{/ 206} \mathrm{~Pb} \\
( \pm 1 \sigma)\end{array}$ & $\begin{array}{c}{ }^{206} \mathrm{~Pb}^{1238} \mathrm{U} \\
( \pm 1 \sigma)\end{array}$ & $\begin{array}{c}{ }^{207} \mathrm{~Pb}^{1206} \mathrm{~Pb} \text { Age } \\
( \pm 1 \sigma \mathrm{Ma})\end{array}$ & $\mathrm{C}(\%)$ \\
\hline \multicolumn{9}{|c|}{ Sample ZM36 : Mununga Quarry Granite (12.6259 S - 31.0434 E } \\
\hline \multicolumn{9}{|c|}{ Analyses conducted during a single session. $14 \mathrm{CZ3}$ standard analyses yielded a $2 \sigma$ error of the mean of $1.11 \%$} \\
\hline ZM36-7 & 0.015 & 568 & 340 & 0.62 & $0.07328 \pm 0.00067$ & $0.16720 \pm 0.00314$ & $1021.7 \pm 18.5$ & 97.6 \\
\hline ZM36-8 & 0.174 & 206 & 164 & 0.82 & $0.07253 \pm 0.00189$ & $0.16960 \pm 0.00379$ & $1000.9 \pm 52.9$ & 100.9 \\
\hline ZM36-9 & 0.081 & 567 & 359 & 0.65 & $0.07358 \pm 0.00069$ & $0.17231 \pm 0.00322$ & $1030.0 \pm 19.0$ & 99.5 \\
\hline ZM36-10 & 0.054 & 615 & 292 & 0.49 & $0.07418 \pm 0.00060$ & $0.17079 \pm 0.00318$ & $1046.4 \pm 16.3$ & 97.1 \\
\hline \multicolumn{9}{|c|}{ Sample CHL5 : Porphyritic granite (12.4491 S - 31.0459 E) } \\
\hline \multicolumn{9}{|c|}{ Analyses conducted during a single session. $7 \mathrm{CZ} 3$ standard analyses yielded a $2 \sigma$ error of the mean of $1.65 \%$} \\
\hline CHL5-1 & 0.506 & 227 & 368 & 1.68 & $0.07432 \pm 0.00129$ & $1.79657 \pm 0.05688$ & $1050.3 \pm 34.9$ & 99.1 \\
\hline CHL5-2 & 0.723 & 269 & 488 & 1.87 & $0.07373 \pm 0.00221$ & $1.69087 \pm 0.07479$ & $1034.0 \pm 60.6$ & 95.9 \\
\hline CHL5-3 & 0.610 & 244 & 44 & 0.18 & $0.07236 \pm 0.00213$ & $1.71635 \pm 0.06805$ & $996.1 \pm 59.9$ & 102.7 \\
\hline CHL5-4 & 0.069 & 130 & 351 & 2.78 & $0.07794 \pm 0.00232$ & $1.94345 \pm 0.07815$ & $1145.3 \pm 59.1$ & 93.6 \\
\hline CHL5-5 & 0.066 & 328 & 586 & 1.85 & $0.07694 \pm 0.00086$ & $1.91776 \pm 0.05474$ & $1119.6 \pm 22.4$ & 95.7 \\
\hline CHL5-6 & 0.547 & 268 & 185 & 0.71 & $0.07340 \pm 0.00218$ & $1.74131 \pm 0.06934$ & $1024.9 \pm 60.2$ & 99.9 \\
\hline CHL5-7 & 0.635 & 421 & 418 & 1.03 & $0.07197 \pm 0.00121$ & $1.65353 \pm 0.05147$ & $985.1 \pm 34.3$ & 100.9 \\
\hline CHL5-8 & 0.582 & 332 & 454 & 1.41 & $0.07228 \pm 0.00165$ & $1.70937 \pm 0.05026$ & $993.7 \pm 46.4$ & 102.7 \\
\hline
\end{tabular}

Sample LW1 : Chilubanama Granite (10.9920 S - 32.3037 E)

Analyses conducted during a single session. $12 \mathrm{CZ3}$ standard analyses yielded a $2 \sigma$ error of the mean of $0.65 \%$

\begin{tabular}{lccccccrr}
\hline LW1-7 & 0.617 & 44 & 54 & 1.27 & $0.09440 \pm 0.00279$ & $0.26390 \pm 0.00411$ & $1516.2 \pm 55.8$ & 99.6 \\
LW1-4 & 0.970 & 57 & 83 & 1.50 & $0.08642 \pm 0.00296$ & $0.25221 \pm 0.00372$ & $1347.5 \pm 66.2$ & 107.6 \\
LW1-2 & 0.392 & 498 & 1001 & 2.08 & $0.09277 \pm 0.00059$ & $0.23907 \pm 0.00232$ & $1483.2 \pm 12.1$ \\
LW1-1 & 0.762 & 87 & 82 & 0.97 & $0.09554 \pm 0.00242$ & $0.27213 \pm 0.00503$ & $1538.8 \pm 47.7$ & 100.8 \\
LW1-5 & 0.282 & 402 & 670 & 1.72 & $0.07268 \pm 0.00077$ & $0.16794 \pm 0.00169$ & $1005.0 \pm 21.4$ \\
LW1-6 & 0.697 & 34 & 51 & 1.56 & $0.09377 \pm 0.00229$ & $0.26772 \pm 0.00450$ & $1503.4 \pm 46.2$ & 101.7 \\
\hline
\end{tabular}

Sample LW2 : Chilubanama Granite (11.0550 S - 32.3299 E)

Analyses conducted during a single session. $12 \mathrm{CZ3}$ standard analyses yielded a $2 \sigma$ error of the mean of $0.65 \%$

\begin{tabular}{lcccccccr}
\hline LW2-1 & 0.334 & 163 & 87 & 0.55 & $0.07036 \pm 0.00135$ & $0.16688 \pm 0.00192$ & $939.0 \pm 39.3$ & 105.9 \\
LW2-2 & 0.844 & 68 & 221 & 3.36 & $0.06581 \pm 0.00287$ & $0.16812 \pm 0.00244$ & $800.5 \pm 91.4$ & 125.1 \\
LW2-9 & 0.307 & 196 & 34 & 0.18 & $0.06984 \pm 0.00124$ & $0.16932 \pm 0.00188$ & $923.6 \pm 36.4$ & 109.2 \\
LW2-3 & 0.036 & 196 & 63 & 0.33 & $0.07220 \pm 0.00090$ & $0.16983 \pm 0.00187$ & $991.7 \pm 25.4$ & 102.0 \\
LW2-4 & 0.120 & 261 & 255 & 1.01 & $0.07078 \pm 0.00077$ & $0.15796 \pm 0.00168$ & $951.2 \pm 22.2$ & 99.4 \\
LW2-10 & 1.293 & 559 & 695 & 1.28 & $0.07170 \pm 0.00202$ & $0.16308 \pm 0.00161$ & $977.5 \pm 57.5$ & 99.6 \\
LW2-5 & 0.138 & 354 & 239 & 0.70 & $0.07042 \pm 0.00065$ & $0.15807 \pm 0.00161$ & $940.7 \pm 18.9$ & 100.6 \\
LW2-6 & 0.099 & 255 & 216 & 0.88 & $0.07085 \pm 0.00063$ & $0.15605 \pm 0.00166$ & $953.1 \pm 18.2$ & 98.1 \\
\hline
\end{tabular}

Sample MTG4 : Chilubanama Granite (10.6636 S - 32.4918 E)

Analyses conducted during a single session. $16 \mathrm{CZ3}$ standard analyses yielded a $2 \sigma$ error of the mean of $0.81 \%$

\begin{tabular}{|c|c|c|c|c|c|c|c|c|}
\hline MTG4-9r & 0.000 & 435 & 9 & 0.02 & $0.07315 \pm 0.00038$ & $0.17075 \pm 0.00255$ & $1018.1 \pm 10.4$ & 99.8 \\
\hline MTG4-10r & 0.000 & 587 & 10 & 0.02 & $0.07289 \pm 0.00042$ & $0.16688 \pm 0.00247$ & $1010.8 \pm 11.7$ & 98.4 \\
\hline MTG4-8r & 0.000 & 577 & 9 & 0.02 & $0.07296 \pm 0.00032$ & $0.17056 \pm 0.00259$ & $1012.9 \pm 9.0$ & 100.2 \\
\hline MTG4-7c & 0.033 & 216 & 131 & 0.63 & $0.12369 \pm 0.00053$ & $0.35853 \pm 0.00550$ & $2010.1 \pm 7.6$ & 98.3 \\
\hline MTG4-10c & 0.082 & 1201 & 26 & 0.02 & $0.07368 \pm 0.00029$ & $0.17054 \pm 0.00250$ & $1032.9 \pm 7.8$ & 98.3 \\
\hline MTG4-9c & 0.235 & 124 & 146 & 1.22 & $0.07099 \pm 0.00103$ & $0.17281 \pm 0.00275$ & $957.1 \pm 29.6$ & 107.4 \\
\hline MTG4-6c & 0.253 & 521 & 63 & 0.12 & $0.07348 \pm 0.00053$ & $0.16841 \pm 0.00250$ & $1027.1 \pm 14.6$ & 97.7 \\
\hline MTG4-4r & 0.265 & 564 & 39 & 0.07 & $0.07130 \pm 0.00053$ & $0.16329 \pm 0.00242$ & $966.0 \pm 15.1$ & 100.9 \\
\hline MTG4-1c & 0.236 & 268 & 29 & 0.11 & $0.12620 \pm 0.00076$ & $0.40044 \pm 0.00685$ & $2045.7 \pm 10.7$ & 106.1 \\
\hline MTG4-4c & 0.656 & 132 & 81 & 0.63 & $0.12419 \pm 0.00118$ & $0.35275 \pm 0.00549$ & $2017.2 \pm 16.8$ & 96.6 \\
\hline MTG4-1r & 1.041 & 522 & 139 & 0.27 & $0.07170 \pm 0.00091$ & $0.16941 \pm 0.00252$ & $977.3 \pm 25.9$ & 103.2 \\
\hline MTG4-8c & 2.478 & 218 & 282 & 1.34 & $0.08996 \pm 0.00222$ & $0.24300 \pm 0.00374$ & $1424.8 \pm 47.0$ & 98.4 \\
\hline MTG4-5c & 4.327 & 624 & 820 & 1.36 & $0.07249 \pm 0.00260$ & $0.14984 \pm 0.00225$ & $999.7 \pm 72.9$ & 90.0 \\
\hline
\end{tabular}

Sample ZM32 : Chilubanama Granite (11.0171 S - 32.0762 E)

Analyses conducted during a single session. $14 \mathrm{CZ3}$ standard analyses yielded a $2 \sigma$ error of the mean of $1.11 \%$

\begin{tabular}{lcccccccc}
\hline ZM32-1 & 0.315 & 268 & 286 & 1.10 & $0.11369 \pm 0.00123$ & $0.31815 \pm 0.00871$ & $1859.3 \pm 19.5$ & 95.8 \\
ZM32-2 & 0.119 & 464 & 404 & 0.90 & $0.10135 \pm 0.00123$ & $0.29651 \pm 0.00809$ & $1649.0 \pm 22.6$ & 101.5 \\
ZM32-3 & 1.024 & 414 & 434 & 1.08 & $0.07062 \pm 0.00173$ & $0.16744 \pm 0.00478$ & $946.4 \pm 50.1$ & 105.5 \\
ZM32-4 & 23.894 & 471 & 393 & 0.86 & $0.08282 \pm 0.00822$ & $0.14496 \pm 0.00408$ & $1265.0 \pm 193.8$ & 69.0 \\
ZM32-5 & 0.042 & 78 & 63 & 0.84 & $0.12553 \pm 0.00216$ & $0.35921 \pm 0.01072$ & $2036.3 \pm 30.5$ & 97.2 \\
ZM32-6 & 0.750 & 90 & 45 & 0.52 & $0.06751 \pm 0.00277$ & $0.16150 \pm 0.00488$ & $853.4 \pm 85.4$ & 113.1 \\
\hline
\end{tabular}

$f_{206}=$ the proportion of common ${ }^{206} \mathrm{~Pb}$ in the total ${ }^{206} \mathrm{~Pb} ; \mathrm{Th} / \mathrm{U}={ }^{232} \mathrm{Th} /{ }^{238} \mathrm{U} ; \% \mathrm{C}=\%$ concordance

All ratios and ages corrected for common $\mathrm{Pb}$ using measured ${ }^{204} \mathrm{~Pb}$ and composition appropriate to the age of the zircon (Stacey and Kramers, 1975) 

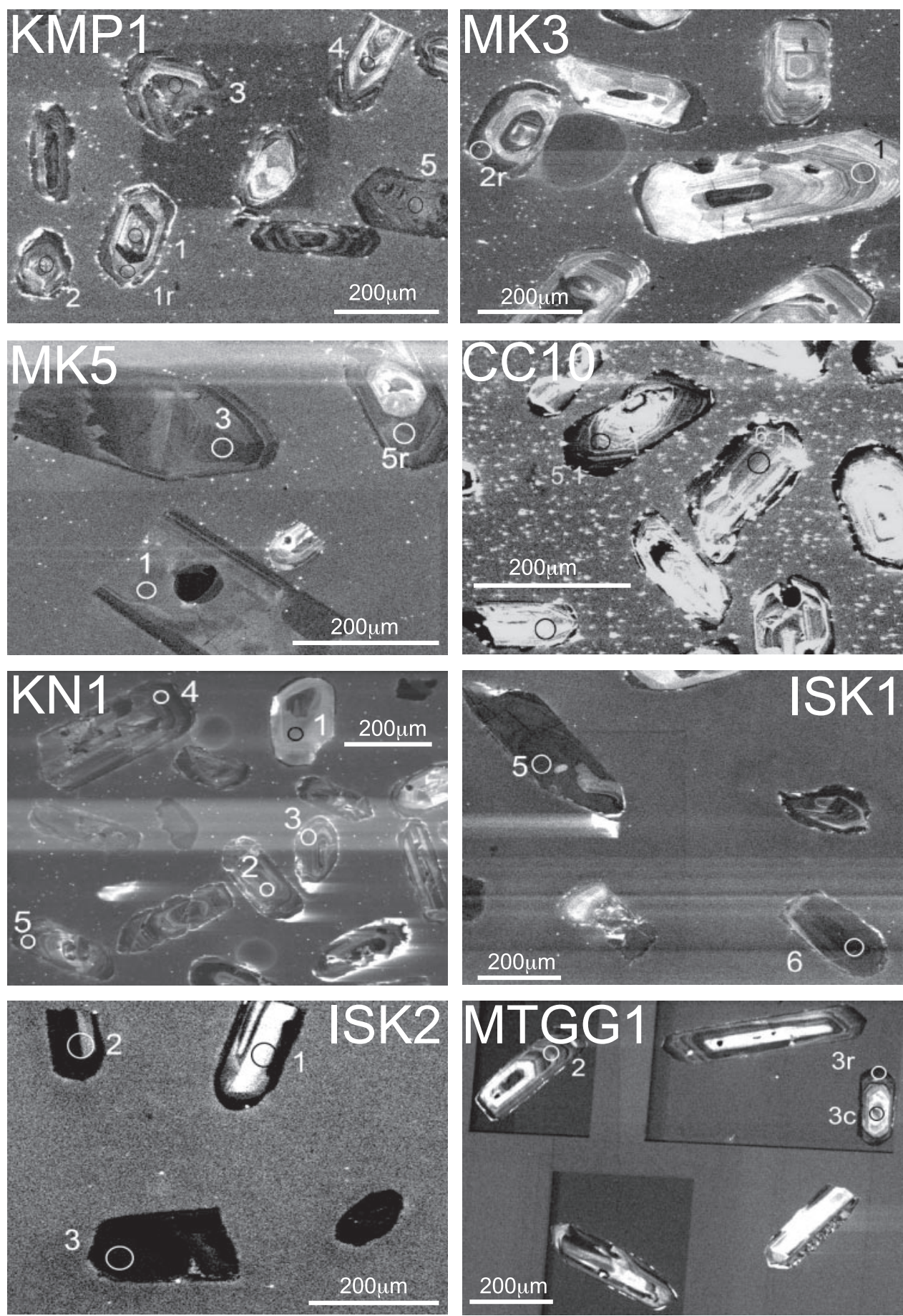

Fig. 2A-E. Cathodo-luminescence images (except sample ZM32 on fig. 2E, which is an optical image) of polished zircons, indicating analyzed spots within the field of view (refer to table 2 for age data). 

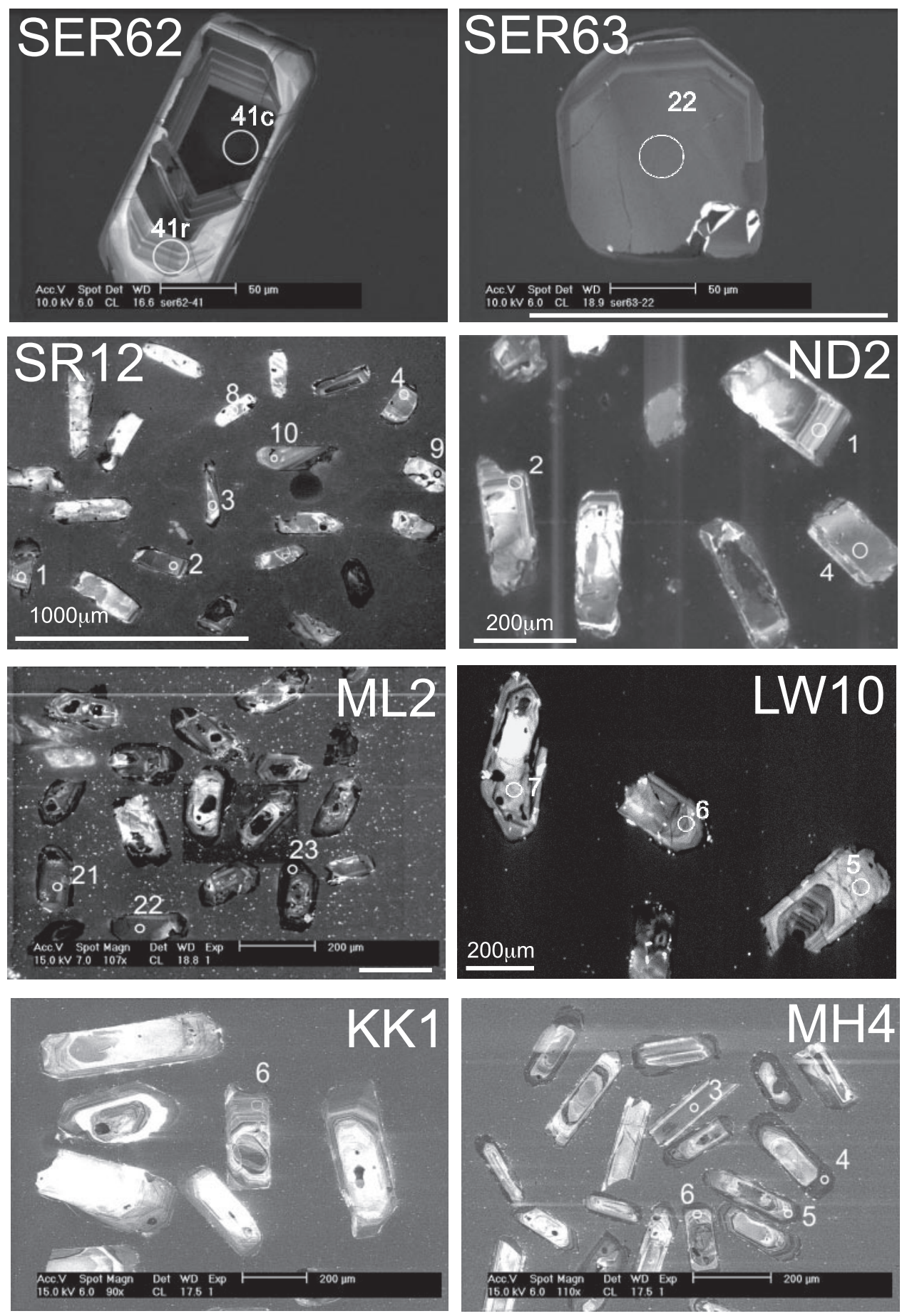

Fig. 2B. 

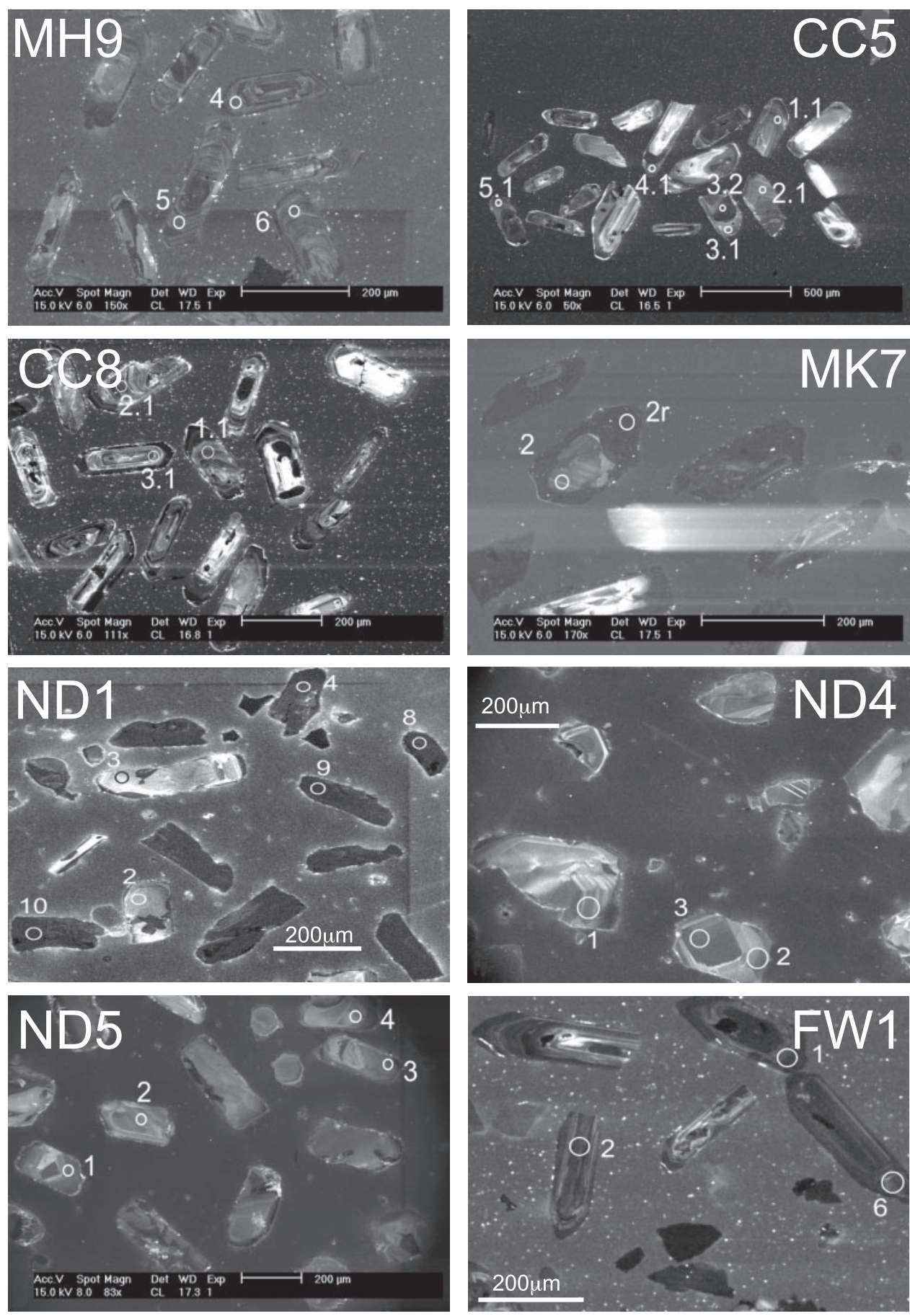

Fig. 2C. 

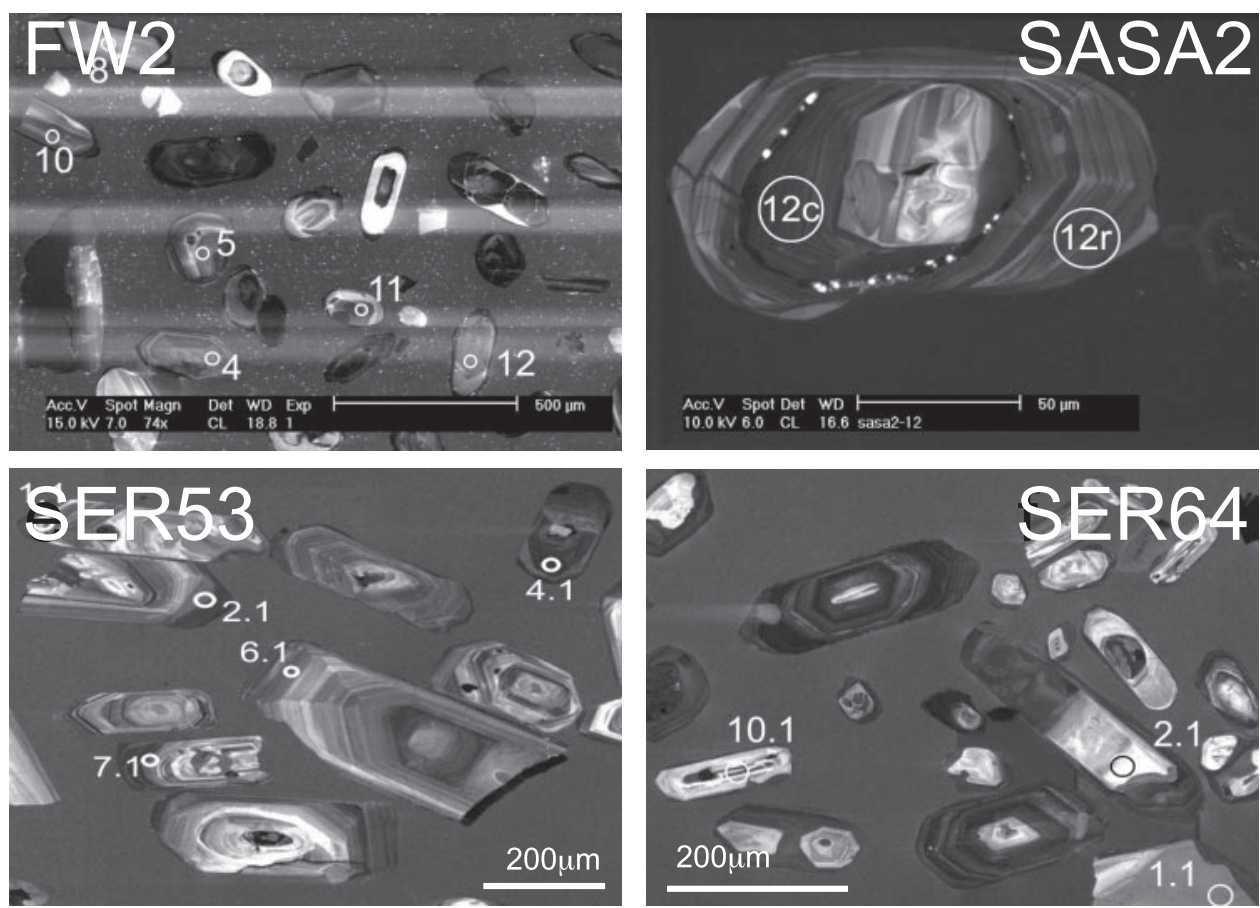

\section{SQG2}

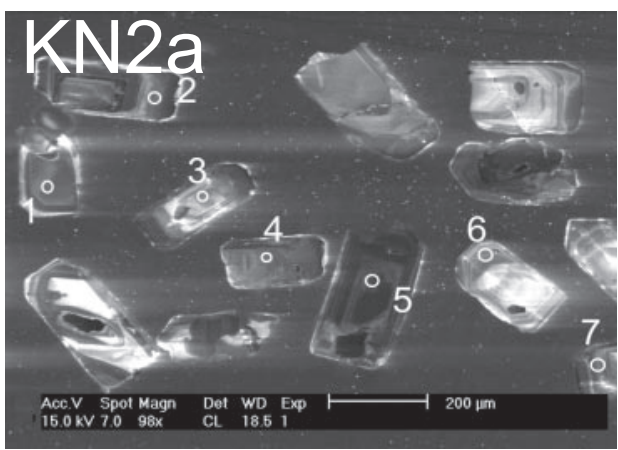

Acc. S Spot Det WD
$10.0 \mathrm{KV} 6.0 \mathrm{CL} \quad 19.0$ SOG2-7
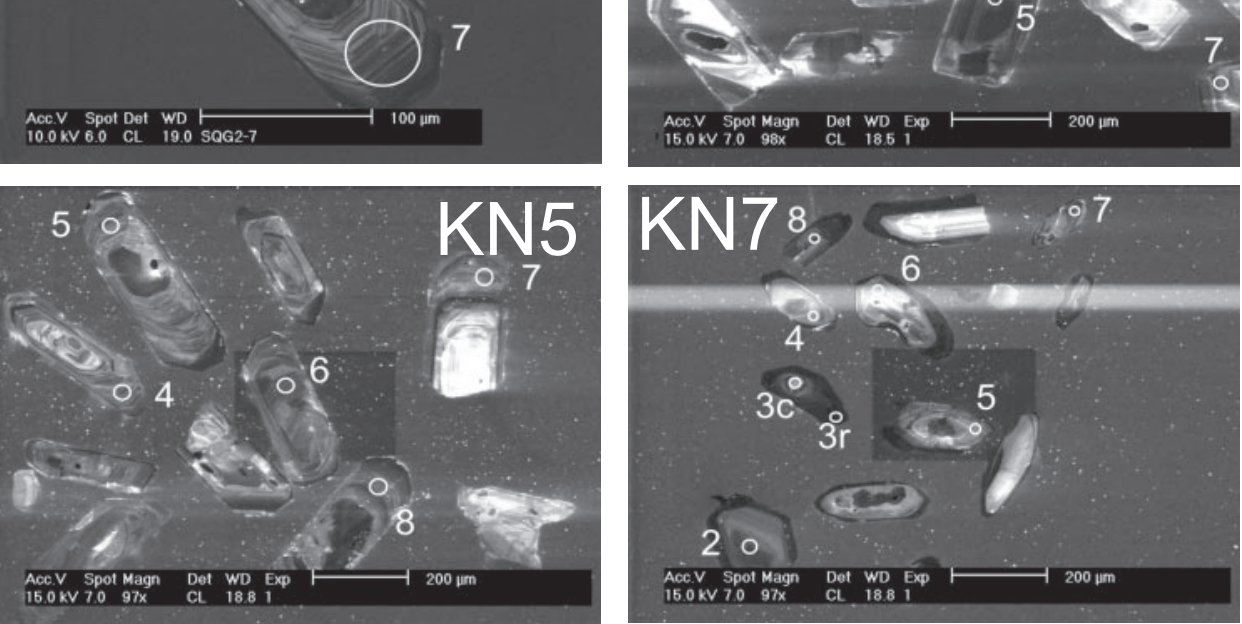

Fig. 2D. 

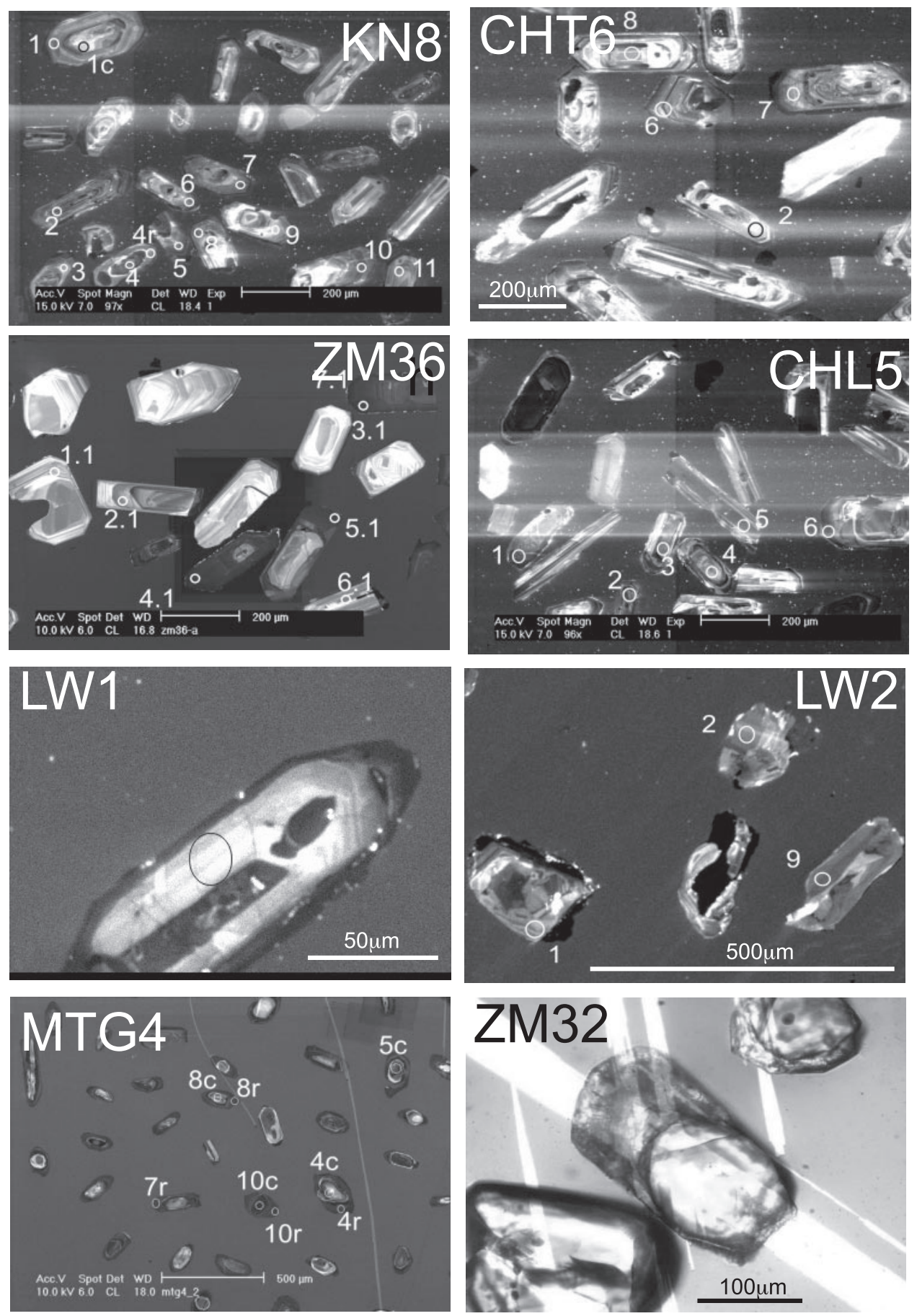

Fig. 2E. 

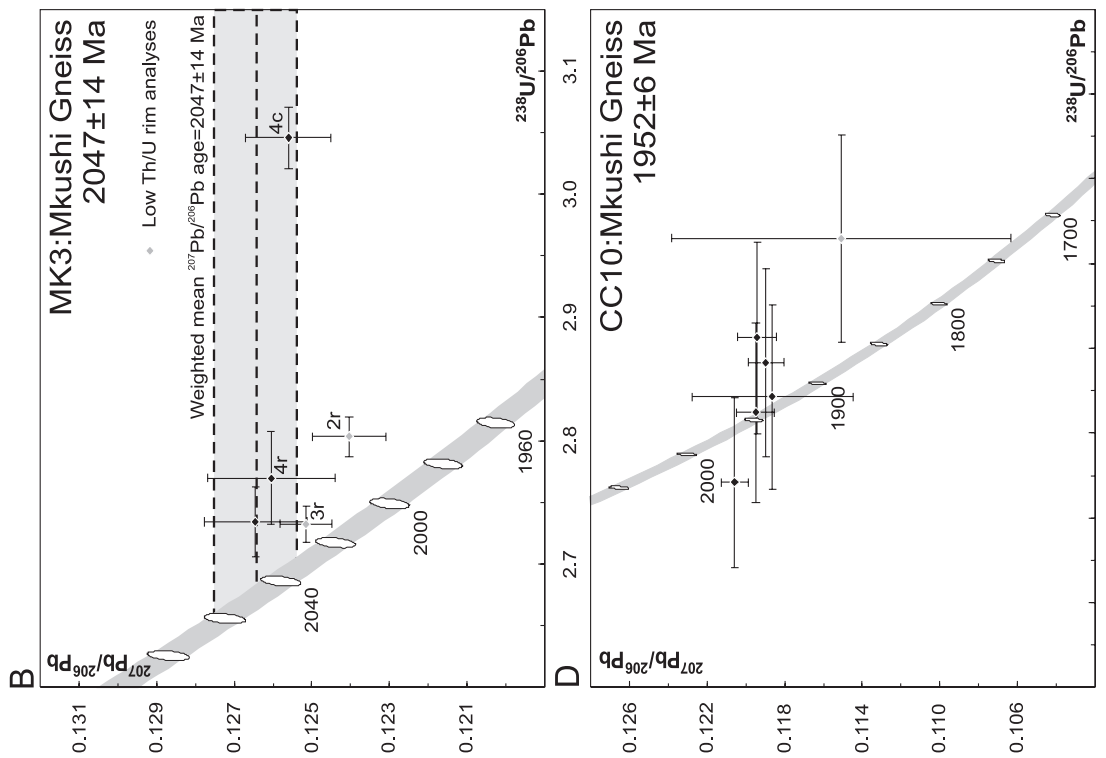

$\stackrel{0}{\circ}$
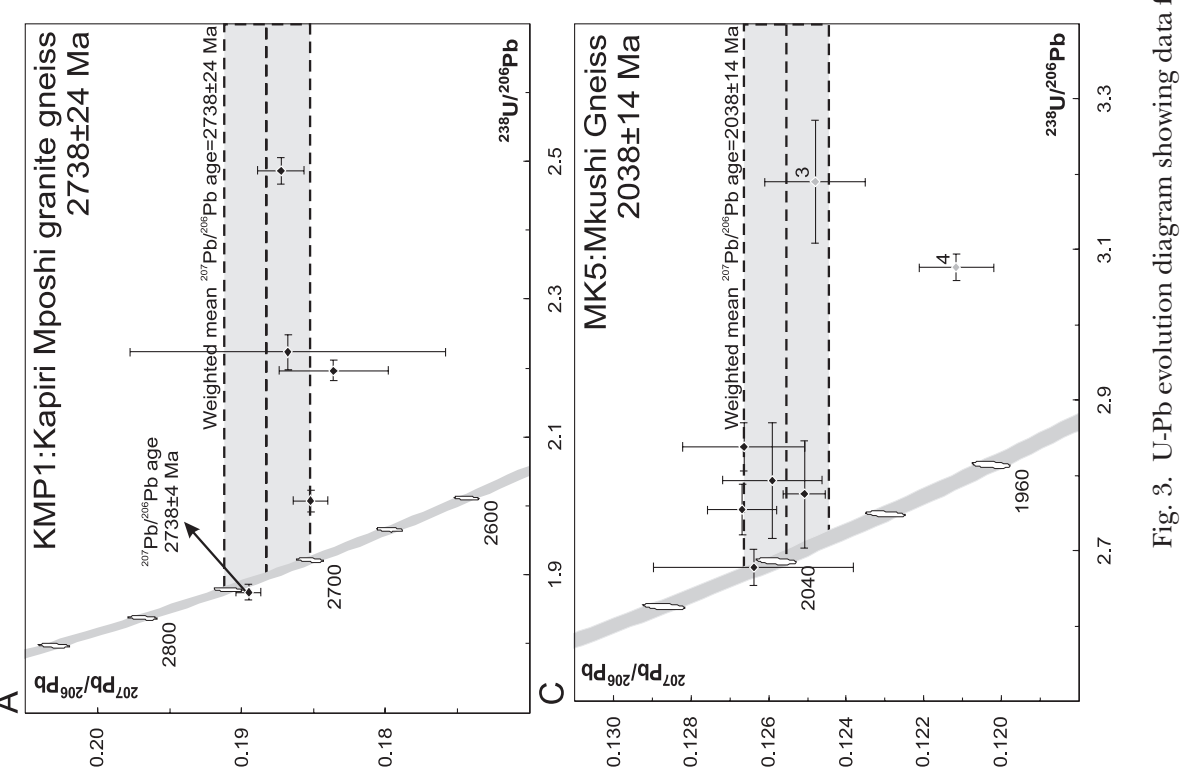
ICP-MS system. The methodology is described in detail by Griffin and others (2000, 2004). Analyses were carried out with a beam diameter of $\sim 40 \mu \mathrm{m}$, a $5 \mathrm{~Hz}$ repetition rate, 60 percent power output and a laser energy of $\sim 0.13 \mathrm{~mJ}$. The ablation times were 100 to 200 seconds. We used the chondritic values of Blichert-Toft and others (1997) for the calculation of $\varepsilon_{\mathrm{Hf}}(\mathrm{t})$ values. Hf model ages $\left(\mathrm{T}_{\mathrm{DM}}\right)$ were calculated based on a depleted-mantle source with $\left({ }^{176} \mathrm{Hf} /{ }^{177} \mathrm{Hf}\right)_{\mathrm{I}}=0.279718$ and ${ }^{176} \mathrm{Hf} /{ }^{177} \mathrm{Hf}=0.0384$. This results in a value of ${ }^{176} \mathrm{Hf} /{ }^{177} \mathrm{Hf}$ of 0.28325 for a mid-ocean ridge basalt at $4.56 \mathrm{Ga}$. $2 \sigma$ errors on the determination of ${ }^{176} \mathrm{Lu} /{ }^{177} \mathrm{Hf}$ isotopic composition were between 1 and 2 percent, which at the ratios obtained here result in an error on $\varepsilon_{\mathrm{Hf}}(\mathrm{t})$ of less than 1 unit.

\section{ZIRCON U-Pb GEOCHRONOLOGY AND Lu-Hf DETERMINATIONS}

\section{Zircon Characteristics}

Zircons from the granitoid bodies are generally large, between 200 and $400 \mu \mathrm{m}$, and euhedral in shape. Length to width ratios vary from equant to 4:1 and most analyzed crystals are colorless to pale yellow in transmitted light, and largely free of large inclusions or cracks. CL imaging brings out distinct oscillatory zoning in all but a few cases, indicative of magmatic crystallization. Only a limited number of granitoid bodies appeared to contain complex zircons, with core and rim domains, which are discussed in detail for those samples. Characteristics of zircon are tabulated in table 1 while CL or optical imagery of representative grains for each sample are shown in figures 2A-E. All U-Pb data are reported in table 2 and Lu-Hf data in table 3.

\section{$\mathrm{U}-\mathrm{Pb}$ and $\mathrm{Lu}$-Hf Results}

The Mkushi Gneiss (samples KMP1, MK3, MK5, CC10 and KN1).-The Mkushi Gneiss was sampled from five different localities (samples KMP1, MK3, MK5, CC10 and KN1, see fig. 1 and table 2). Sample KMP1 was collected from a strongly foliated granite gneiss in a quarry along the sealed road north of Kapiri-Mposhi. Samples MK3 and MK5 were taken from biotite granite gneiss, and pinkish biotite granite gneiss in Munshiwemba Quarry south of Mkushi (MG, fig. 1B). Samples CC10 and KN1 were collected from biotite granite gneiss further east. Munshiwemba Quarry is the type locality, and offers excellent exposure of the Mkushi Gneiss, which is cross-cut by numerous large aplite dikes and pegmatites, most of which contain abundant chalcopyrite. At least two varieties of the Mkushi Gneiss occur in the quarry. The dominant variant is a foliated pink biotite granite gneiss (sample MK5) which locally gives way to a subordinant, highly sheared, strongly foliated dark gray biotite gneiss (sample MK3). Zircon from a sample of the biotite granite gneiss as well as the aplites in the quarry was previously dated by Rainaud and others (2002, 2005) using the U-Pb SHRIMP technique and yielded crystallization ages of $2049 \pm 6 \mathrm{Ma}$ (gneiss) and $2036 \pm 22 \mathrm{Ma}$ (aplite) respectively. A later magmatic event is recognized in magmatic rim overgrowths on zircon from the aplite, which give a poorly constrained age of $1088 \pm 159 \mathrm{Ma}$.

Sample KMP1-Six analyses were conducted on five zircon grains (fig. 3A, table 2). $\mathrm{Th} / \mathrm{U}$ ratios range between 0.3 and 0.7 , with $\mathrm{U}+\mathrm{Th}$ content between 430 and 1050 $\mathrm{ppm}$. One data point recorded high non-radiogenic lead (common $\mathrm{Pb}$ or $\mathrm{Pb}_{\mathrm{c}}$ ) and defines an imprecise ${ }^{207} \mathrm{~Pb} /{ }^{206} \mathrm{~Pb}$ age of $3105 \pm 143 \mathrm{Ma}$, possibly indicating a Mesoarchean xenocrystic component. The remaining data record variable $\mathrm{Pb}_{\mathrm{c}}$ and $\mathrm{Pb}$-loss, positively correlated with $\mathrm{U}$ and Th content. A broad trend parallel to the ${ }^{238} \mathrm{U} /{ }^{206} \mathrm{~Pb}$ axis indicates that the majority of $\mathrm{Pb}$ was lost in recent times. A weighted mean ${ }^{207} \mathrm{~Pb} /{ }^{206} \mathrm{~Pb}$ age of $2723 \pm 24 \mathrm{Ma}$ [MSWD (Mean Square of Weighted Deviates $)=9.3$ ] can be calculated from the data. The most concordant data point, which also records the lowest amount of $\mathrm{Pb}_{\mathrm{c}}$, has a ${ }^{207} \mathrm{~Pb} /{ }^{206} \mathrm{~Pb}$ age of $2738 \pm 8 \mathrm{Ma}$, within error of the weighted mean age. Although the data are inadequate to unequivocally 
TABLE 3

Laser Ablation ICP-MS Lu-Hf data for zircon from granitoid rocks in the Irumide Belt

\begin{tabular}{|c|c|c|c|c|c|c|c|c|c|}
\hline \multirow{2}{*}{$\begin{array}{l}\text { Analysis } \\
\text { No. } \\
\text { SQG-01 }\end{array}$} & \multicolumn{2}{|c|}{$\begin{array}{l}{ }^{176} \mathrm{Hf} /{ }^{177} \mathrm{Hf} \\
1 \sigma \text { abs }\end{array}$} & \multicolumn{3}{|c|}{$\begin{array}{c}{ }^{176} \mathrm{Lu} /{ }^{177} \mathrm{Hf}^{176} \mathrm{Yb} /{ }^{177} \mathrm{Hf} \\
{ }^{176} \mathrm{Hf} /{ }^{177} \mathrm{Hf} \\
\text { initial }\end{array}$} & $\begin{array}{c}\varepsilon_{\mathrm{Hf}}(\mathrm{t}) \\
1 \sigma \\
\end{array}$ & \multicolumn{2}{|c|}{$\begin{array}{l}\text { T(DM) Hf Chur (t) } \\
\text { (Ga) }\end{array}$} & Hf DM (t) \\
\hline & 0.281817 & 0.000010 & $\overline{0.001464}$ & 0.057735 & \begin{tabular}{c|}
0.281788 \\
\end{tabular} & 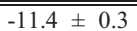 & 1.97 & 0.282109 & 0.282485 \\
\hline SQG-02 & 0.281752 & 0.000011 & 0.000896 & 0.037813 & 0.281734 & $-13.3 \pm 0.4$ & 2.03 & 109 & 282485 \\
\hline SQG-03 & 0.281876 & 0.000013 & 0.001985 & 0.088863 & 0.281836 & $-9.7 \pm 0.5$ & 1.92 & 0.282109 & 0.282485 \\
\hline SQG-04 & 0.281776 & 0.000010 & 0.001293 & 0.059384 & 0.281750 & $-12.7 \pm 0.3$ & 2.02 & 0.282109 & 0.282485 \\
\hline SQG-05 & 0.281770 & 0.000007 & 0.000906 & 0.039462 & 0.281752 & $-12.7 \pm 0.3$ & 2.01 & 0.282109 & 0.282485 \\
\hline SQG-06 & 0.281964 & 0.000010 & 0.002702 & 0.124003 & 0.281910 & $-7.1 \pm 0.4$ & 1.83 & 0.282109 & 0.282485 \\
\hline SQG-07 & 0.281825 & 0.000012 & 0.001061 & 0.047872 & 0.281804 & $-10.8 \pm 0.4$ & 1.94 & 0.282109 & 0.282485 \\
\hline SQG-08 & 0.282020 & 0.000013 & 0.003313 & 0.156771 & 0.281954 & $-5.5 \pm 0.5$ & 1.79 & & 0.282485 \\
\hline SQG-09 & 0.281880 & 0.000013 & 0.002382 & 0.107775 & 0.281832 & $-9.8 \pm 0.5$ & 1.93 & & 0.282485 \\
\hline SQG-10 & 0.281807 & 0.000008 & 0.000749 & 0.031837 & 0.281792 & $-11.2 \pm 0.3$ & 1.95 & & 0.282485 \\
\hline SQG-11 & 0.281771 & 0.000011 & 0.001187 & 0.049366 & 0.281747 & $-12.8 \pm 0.4$ & 2.02 & & 0.282485 \\
\hline SQG-12 & 0.281774 & 0.000011 & 0.001252 & 0.054246 & 0.281749 & $-12.8 \pm 0.4$ & 2.02 & 0.282109 & 0.282485 \\
\hline \multicolumn{3}{|l|}{ Median value } & & & & $-11.3 \pm 0.4$ & 1.96 & & \\
\hline ZM36-01 & 0.281682 & 0.000008 & 0.000616 & 0.028817 & 0.281670 & $-15.6 \pm 0.3$ & 2.11 & 09 & 484 \\
\hline M36-02 & 0.281659 & 0.000010 & 0.000630 & 0.028771 & 0.281646 & $-16.4 \pm 0.4$ & 2.14 & & \\
\hline M36-03 & 0.281728 & 0.000013 & 0.000807 & 0.038463 & 0.281712 & $-14.1 \pm 0.5$ & 06 & & \\
\hline M36-04 & 0.281740 & 0.000012 & 0.001054 & 0.043792 & 0.281719 & $-13.8 \pm 0.4$ & 05 & & \\
\hline 5 & 0.281671 & 0.000011 & 0.000747 & 0.034156 & 0.281656 & $-16.0 \pm 0.4$ & 13 & & \\
\hline ZM3 & 0.281705 & 0.000017 & 0.000757 & 0.035435 & 0.281690 & $-14.8 \pm 0.6$ & 2.08 & & \\
\hline ZM36-07 & 0.281486 & 0.000011 & 0.001495 & 0.067239 & 0.281456 & $-23.1 \pm 0.4$ & 2.42 & & 484 \\
\hline ZM3 & 0.281796 & 0.000011 & 0.000559 & 0.023304 & 0.281785 & $-11.5 \pm 0.4$ & 1.95 & 09 & 484 \\
\hline ZM36-09 & 0.281748 & 0.000008 & 0.002079 & 0.097359 & 0.281706 & $-14.3 \pm 0.3$ & 10 & 09 & 484 \\
\hline ZM36-10 & 0.281705 & 0.000006 & 0.000674 & 0.030041 & 0.281692 & $-14.8 \pm 0.2$ & .08 & & 484 \\
\hline ZM3 & 81727 & 0.000010 & 0.000800 & 0.037578 & 0.281711 & $-14.1 \pm 0.3$ & .06 & & 184 \\
\hline ZM36-12 & 0.281719 & 0.00 & 0.000746 & 0.034606 & 0.281704 & $-14.3 \pm 0.3$ & 2.07 & 09 & 0.2 \\
\hline \multicolumn{3}{|l|}{ Median value } & & & & $-14.6 \pm 0.4$ & 2.08 & & \\
\hline MH9C-01 & 0.281754 & 0.000006 & 0.000250 & 0.008254 & 0.281749 & $-12.7 \pm 0.2$ & 1.99 & 106 & 0.282481 \\
\hline MHs & 0.281738 & 0.000010 & 0.000639 & 0.024921 & 0.281725 & $-13.5 \pm 0.3$ & 2.03 & 06 & 481 \\
\hline MHS & 0.281830 & 0.000012 & 0.001687 & 0.075506 & 0.281796 & $-11.0 \pm 0.4$ & 1.97 & & 481 \\
\hline MH & 0.281727 & 0.000008 & 0.000492 & 0.022538 & 0.281717 & $-13.8 \pm 0.3$ & 2.04 & 06 & 481 \\
\hline & 0.281764 & 0.000009 & 0.000700 & 0.031753 & 0.281750 & $-12.6 \pm 0.3$ & 2.00 & & 481 \\
\hline & 0.281766 & 0.000008 & 0.000860 & 0.040138 & 0.281749 & $-12.7 \pm 0.3$ & 2.01 & & 481 \\
\hline & 81703 & 0.000012 & 0.000525 & 0.024316 & 0.281692 & $-14.7 \pm 0.4$ & 2.07 & & 81 \\
\hline MH9C-08 & 0.281801 & 0.000012 & 0.000986 & 0.039468 & 0.281 & $-11.5 \pm 0.4$ & 1.97 & 106 & 0.282481 \\
\hline \multicolumn{3}{|l|}{ Median value } & & & & $-12.7 \pm 0.3$ & 2.01 & & \\
\hline KN2A-01 & 0.281755 & 0.000009 & 0.001216 & 0.057286 & 0.281731 & $-13.3 \pm 0.3$ & 2.04 & 105 & 479 \\
\hline $\mathrm{KN} 2 \mathrm{~A}-02$ & 0.281738 & 0.000009 & 0.000696 & 0.031617 & 0.281724 & $-13.5 \pm 0.3$ & 2.04 & 05 & 479 \\
\hline $\mathrm{KN} 2 \mathrm{~A}-03$ & 0.281725 & 0.000010 & 0.000450 & 0.020186 & 0.281716 & $-13.8 \pm 0.3$ & 2.04 & 105 & 479 \\
\hline $\mathrm{KN} 2 \mathrm{~A}-04$ & 0.281726 & 0.000008 & 0.000539 & 0.024229 & 0.281715 & $-13.8 \pm 0.3$ & 2.04 & 105 & 479 \\
\hline $\mathrm{KN} 2 \mathrm{~A}-05$ & 0.281708 & 0.000013 & 0.000888 & 0.042241 & 0.281690 & $-14.7 \pm 0.5$ & 2.09 & 105 & 479 \\
\hline KN2A-06 & 0.281708 & 0.000008 & 0.000739 & 0.032807 & 0.281693 & -14.6 & 2.08 & 2105 & 0.282479 \\
\hline KN2A-07 & 0.281748 & 0.000009 & 0.001241 & 0.056824 & 0.281723 & $-13.5 \pm 0.3$ & 2.05 & 2105 & 0.282479 \\
\hline KN2A-08 & 0.281701 & 0.000012 & 0.000588 & 0.027078 & 0.281689 & $-14.7 \pm 0.4$ & 2.08 & 0.282105 & 0.282479 \\
\hline \multicolumn{3}{|l|}{ Median value } & & & & $-13.8 \pm 0.3$ & 2.05 & & \\
\hline ND4-01 & 0.281796 & 0.000009 & 0.000706 & 0.027257 & 0.281782 & $-11.4 \pm 0.3$ & & 0.282105 & 0.282479 \\
\hline ND4-02 & 0.281746 & 0.000013 & 0.000529 & 0.020183 & 0.281735 & $-13.1 \pm 0.5$ & 2.02 & 0.282105 & 0.282479 \\
\hline ND4-03 & 0.281826 & 0.000009 & 0.003856 & 0.173260 & 0.281749 & $-12.6 \pm 0.3$ & 2.09 & 0.282105 & 0.282479 \\
\hline ND4-04 & 0.281819 & 0.000014 & 0.002492 & 0.110076 & 0.281769 & $-11.9 \pm 0.5$ & 2.02 & 0.282105 & 0.282479 \\
\hline ND4-05 & 0.281810 & 0.000010 & 0.001114 & 0.044241 & 0.281788 & $-11.2 \pm 0.4$ & 1.96 & 0.282105 & 0.282479 \\
\hline ND4-06 & 0.281763 & 0.000010 & 0.000858 & 0.034262 & 0.281746 & $-12.7 \pm 0.4$ & 2.01 & 0.282105 & 0.282479 \\
\hline ND4-07 & 0.281802 & 0.000011 & 0.000929 & 0.035891 & 0.281783 & $-11.4 \pm 0.4$ & 1.96 & 0.282105 & 0.282479 \\
\hline ND4-08 & 0.281807 & 0.000009 & 0.000793 & 0.029889 & 0.281791 & $-11.1 \pm 0.3$ & 1.95 & 0.282105 & 0.282479 \\
\hline ND4-09 & 0.281812 & 0.000011 & 0.000598 & 0.022854 & 0.281800 & $-10.8 \pm 0.4$ & 1.93 & 0.282105 & 0.282479 \\
\hline ND4-10 & 0.281780 & 0.000009 & 0.000699 & 0.027245 & 0.281766 & $-12.0 \pm 0.3$ & 1.98 & 0.282105 & 0.282479 \\
\hline \multicolumn{3}{|c|}{ Median value } & & & & $-11.7 \pm 0.4$ & 1.98 & & \\
\hline
\end{tabular}


TABLE 3

(continued)

\begin{tabular}{|c|c|c|c|c|c|c|c|c|c|}
\hline \multirow{2}{*}{$\begin{array}{l}\text { Analysis } \\
\text { No. } \\
\text { ML2-01 }\end{array}$} & \multicolumn{2}{|c|}{$\begin{array}{c}{ }^{176} \mathbf{H f} /{ }^{177} \mathbf{H f} \\
1 \sigma \text { abs }\end{array}$} & \multicolumn{3}{|c|}{$\begin{array}{c}{ }^{176} \mathrm{Lu} /{ }^{177} \mathrm{Hf}^{176} \mathrm{Yb} /{ }^{177} \mathrm{Hf} \\
{ }^{176} \mathrm{Hf} / \\
\text { initial }\end{array}$} & $\begin{array}{c}\varepsilon_{\mathrm{Hf}}(\mathrm{t}) \\
1 \sigma\end{array}$ & \multicolumn{2}{|c|}{$\begin{array}{l}\text { T(DM) Hf Chur (t) } \\
\text { (Ga) }\end{array}$} & Hf DM (t) \\
\hline & 0.281796 & 0.000015 & 0.000900 & 0.036899 & 0.281769 & $0.2 \pm 0.5$ & 1.97 & 0.281763 & 0.282084 \\
\hline ML2-02 & 0.281814 & 0.000012 & 0.001152 & 0.056596 & 0.281779 & $0.6 \pm 0.4$ & 1.96 & 0.281763 & 0.282084 \\
\hline ML2-03 & 0.281831 & 0.000011 & 0.001381 & 0.057970 & 0.281789 & $0.9 \pm 0.4$ & 1.95 & 0.281763 & 0.282084 \\
\hline ML2-04 & 0.281848 & 0.000007 & 0.001211 & 0.057235 & 0.281811 & $1.7 \pm 0.3$ & 1.92 & 0.281763 & 0.282084 \\
\hline ML2-05 & 0.281830 & 0.000011 & 0.001165 & 0.058746 & 0.281795 & $1.1 \pm 0.4$ & 1.94 & 0.281763 & 0.282084 \\
\hline ML2-06 & 0.281794 & 0.000010 & 0.000979 & 0.046523 & 0.281764 & $0.0 \pm 0.4$ & 1.98 & .281763 & 0.282084 \\
\hline ML2-07 & 0.281856 & 0.000009 & 0.001295 & 0.064138 & 0.281817 & $1.9 \pm 0.3$ & 1.91 & 281763 & 0.282084 \\
\hline ML2-08 & 0.281833 & 0.000015 & 0.001312 & 0.061822 & 0.281793 & $1.1 \pm 0.5$ & 1.94 & 281763 & 0.282084 \\
\hline ML2-09 & 0.281926 & 0.000013 & 0.000841 & 0.037107 & 0.281900 & $4.9 \pm 0.5$ & 1.80 & 281763 & 0.282084 \\
\hline ML2-10 & 0.282034 & 0.000011 & 0.003592 & 0.168602 & 0.281925 & $5.7 \pm 0.4$ & 1.78 & 0.281763 & 0.282084 \\
\hline \multicolumn{3}{|l|}{ Median value } & & & & $1.1 \pm 0.4$ & 1.94 & & \\
\hline LW10-01 & 0.281592 & 0.000009 & 0.000628 & 0.029195 & 0.281572 & $-5.4 \pm 0.3$ & 2.23 & 0.281724 & 0.282039 \\
\hline LW10-02 & 0.281650 & 0.000019 & 0.000628 & 0.028650 & 0.281630 & $-3.3 \pm 0.7$ & 2.15 & 724 & 82039 \\
\hline LW10-03 & 0.281518 & 0.000009 & 0.000702 & 0.032742 & 0.281496 & $-8.1 \pm 0.3$ & 2.33 & 724 & 2039 \\
\hline LW10-04 & 0.281611 & 0.000009 & 0.000687 & 0.031814 & 0.281589 & $-4.8 \pm 0.3$ & 2.20 & 24 & 2039 \\
\hline LW10-05 & 0.281618 & 0.000010 & 0.000928 & 0.036091 & 0.281589 & $-4.8 \pm 0.3$ & 2.21 & 724 & 2039 \\
\hline LW10-06 & 0.281598 & 0.000009 & 0.000732 & 0.034011 & 0.281575 & $-5.3 \pm 0.3$ & 2.22 & 724 & 2039 \\
\hline LW10-07 & 0.281663 & 0.000011 & 0.000845 & 0.037824 & 0.281636 & $-3.1 \pm 0.4$ & 2.14 & 724 & 2039 \\
\hline LW10-08 & 0.281634 & 0.000014 & 0.001734 & 0.067302 & 0.281579 & $-5.1 \pm 0.5$ & 2.23 & 724 & 2039 \\
\hline LW10-09 & 0.281318 & 0.000016 & 0.001185 & 0.057261 & 0.281281 & $-15.7 \pm 0.6$ & 2.62 & 724 & 2039 \\
\hline LW10-10 & 0.281650 & 0.000013 & 0.000975 & 0.042360 & 0.281619 & $-3.7 \pm 0.5$ & 2.17 & 724 & 0.282039 \\
\hline LW10-11 & 0.281567 & 0.000011 & 0.000592 & 0.027823 & 0.281548 & $-6.2 \pm 0.4$ & 2.26 & 281724 & 0.282039 \\
\hline LW10-12 & 0.281620 & 0.000012 & 0.000753 & 0.035998 & 0.281596 & $-4.5 \pm 0.4$ & 2.20 & .281724 & 0.282039 \\
\hline LW10-13 & 0.281596 & 0.000010 & 0.000875 & 0.041062 & 0.281568 & $-5.5 \pm 0.4$ & 2.23 & 0.281724 & 0.282039 \\
\hline LW10-14 & 0.281576 & 0.000008 & 0.000792 & 0.038365 & 0.281551 & $-6.1 \pm 0.3$ & 2.26 & 0.281724 & 0.282039 \\
\hline \multicolumn{3}{|l|}{ Median value } & & & & $-5.2 \pm 0.4$ & 2.22 & & \\
\hline ND2-01 & 0.281879 & 0.000047 & 0.002740 & 0.105585 & 0.281792 & $2.8 \pm 1.6$ & 1.95 & 13 & 2026 \\
\hline ND2-02 & 0.281726 & 0.000013 & 0.001175 & 0.051600 & 0.281689 & $-0.9 \pm 0.5$ & 2.08 & 13 & 2026 \\
\hline ND2-03 & 0.281866 & 0.000014 & 0.000621 & 0.020406 & 0.281846 & $4.7 \pm 0.5$ & 1.86 & 713 & 0.282026 \\
\hline ND2-04 & 0.281775 & 0.000022 & 0.003093 & 0.118518 & 0.281676 & $-1.3 \pm 0.8$ & 2.12 & .281713 & 0.282026 \\
\hline ND2-05 & 0.281780 & 0.000015 & 0.000871 & 0.042136 & 0.281752 & $1.4 \pm 0.5$ & 1.99 & .281713 & 0.282026 \\
\hline ND2-06 & 0.281729 & 0.000012 & 0.000898 & 0.043506 & 0.281700 & $-0.4 \pm 0.4$ & 2.06 & .281713 & 0.282026 \\
\hline ND2-07 & 0.281722 & 0.000024 & 0.001640 & 0.070276 & 0.281670 & -1.5 & 2.11 & .281713 & 0.282026 \\
\hline ND2-08 & 0.281780 & 0.000014 & 0.001014 & 0.047495 & 0.281748 & $1.2 \pm 0.5$ & 2.00 & 0.281713 & 0.282026 \\
\hline \multicolumn{3}{|l|}{ Median value } & & & & $0.4 \pm 0.7$ & 2.03 & & \\
\hline SER63-01 & 0.282077 & 0.000012 & 0.004451 & 0.211394 & 0.281933 & $8.4 \pm 0.4$ & 1.76 & 96 & 2007 \\
\hline SER63-02 & 0.281932 & 0.000009 & 0.003912 & 0.181492 & 0.281805 & $3.9 \pm 0.3$ & 1.94 & 596 & 0.282007 \\
\hline SER63-03 & 0.281829 & 0.000008 & 0.001761 & 0.081006 & 0.281772 & $2.7 \pm 0.3$ & 1.97 & .281696 & 0.282007 \\
\hline SER63-04 & 0.281846 & 0.000008 & 0.001447 & 0.066983 & 0.281799 & $3.6 \pm 0.3$ & 1.93 & 0.281696 & 0.282007 \\
\hline SER63-05 & 0.281718 & 0.000007 & 0.001002 & 0.047427 & 0.281686 & $-0.4 \pm 0.3$ & 2.08 & 0.281696 & 0.282007 \\
\hline SER63-06 & 0.281807 & 0.000028 & 0.001582 & 0.080215 & 0.281756 & $2.1 \pm 1.0$ & 1.99 & 0.281696 & 0.282007 \\
\hline SER63-07 & 0.281778 & 0.000009 & 0.001594 & 0.075933 & 0.281726 & $1.1 \pm 0.3$ & 2.03 & 0.281696 & 0.282007 \\
\hline SER63-08 & 0.281767 & 0.000014 & 0.001209 & 0.058797 & 0.281728 & $1.1 \pm 0.5$ & 2.03 & 0.281696 & 0.282007 \\
\hline SER63-09 & 0.281835 & 0.000013 & 0.001816 & 0.090178 & 0.281776 & $2.8 \pm 0.5$ & 1.97 & 0.281696 & 0.282007 \\
\hline SER63-10 & 0.282030 & 0.000012 & 0.001780 & 0.089395 & 0.281972 & $9.8 \pm 0.4$ & 1.70 & 0.281696 & 0.282007 \\
\hline SER63-11 & 0.281825 & 0.000011 & 0.001765 & 0.084841 & 0.281768 & $2.5 \pm 0.4$ & 1.98 & 0.281696 & 0.282007 \\
\hline SER63-12 & 0.282027 & 0.000013 & 0.001159 & 0.047383 & 0.281989 & $10.4 \pm 0.5$ & 1.67 & 0.281696 & 0.282007 \\
\hline \multicolumn{3}{|l|}{ Median value } & & & & $2.8 \pm 0.4$ & 1.97 & & \\
\hline SER6-2C-01 & 0.281705 & 0.000010 & 0.001163 & 0.055492 & 0.281667 & $-0.8 \pm 0.3$ & 2.11 & 0.281688 & 0.281998 \\
\hline SER6-2C-02 & 0.281691 & 0.000013 & 0.000662 & 0.031210 & 0.281669 & $-0.7 \pm 0.5$ & 2.10 & 0.281688 & 0.281998 \\
\hline SER6-2C-03 & 0.281751 & 0.000011 & 0.002750 & 0.115877 & 0.281661 & $-1.0 \pm 0.4$ & 2.13 & 0.281688 & 0.281998 \\
\hline SER6-2C-04 & 0.281727 & 0.000014 & 0.001009 & 0.048149 & 0.281694 & $0.2 \pm 0.5$ & 2.07 & 0.281688 & 0.281998 \\
\hline SER6-2C-05 & 0.281665 & 0.000009 & 0.000533 & 0.023983 & 0.281648 & $-1.5 \pm 0.3$ & 2.12 & 0.281688 & 0.281998 \\
\hline SER6-2C-06 & 0.281696 & 0.000010 & 0.000667 & 0.030818 & 0.281674 & $-0.5 \pm 0.4$ & 2.09 & 0.281688 & 0.281998 \\
\hline SER6-2C-07 & 0.281652 & 0.000008 & 0.000596 & 0.026716 & 0.281633 & $-2.0 \pm 0.3$ & 2.15 & 0.281688 & 0.281998 \\
\hline SER6-2C-08 & 0.281709 & 0.000011 & 0.001083 & 0.051674 & 0.281674 & $-0.5 \pm 0.4$ & 2.10 & 0.281688 & 0.281998 \\
\hline \multicolumn{3}{|l|}{ Median value } & & & & $-0.7 \pm 0.4$ & 2.10 & & \\
\hline
\end{tabular}


TABLE 3

(continued)

\begin{tabular}{|c|c|c|c|c|c|c|c|c|c|}
\hline \multirow{2}{*}{$\begin{array}{l}\text { Analysis } \\
\text { No. } \\
\text { ISK1-01 }\end{array}$} & \multicolumn{2}{|c|}{$\begin{array}{l}{ }^{176} \mathrm{Hf} /{ }^{177} \mathrm{Hf} \\
1 \sigma \text { abs }\end{array}$} & \multicolumn{3}{|c|}{$\begin{array}{c}{ }^{176} \mathrm{Lu} /{ }^{177} \mathrm{Hf}{ }^{176} \mathrm{Yb} /{ }^{177} \mathrm{Hf}^{176} \mathrm{Hf} /{ }^{177} \mathrm{Hf} \\
\text { initial }\end{array}$} & $\begin{array}{c}\mathcal{E}_{\mathrm{Hf}}(t) \\
1 \sigma \\
\end{array}$ & \multicolumn{2}{|c|}{$\begin{array}{l}\text { T(DM) Hf Chur (t) } \\
\text { (Ga) }\end{array}$} & Hf DM (t) \\
\hline & 0.281569 & 0.000007 & 0.001009 & 0.045046 & 0.281530 & $0.9 \pm 0.2$ & 2.28 & 0.281504 & 0.281784 \\
\hline ISK1-02 & 0.281605 & 0.000009 & 0.000599 & 0.025713 & 0.281582 & $2.8 \pm 0.3$ & 2.21 & 0.281504 & 0.281784 \\
\hline ISK1-03 & 0.281555 & 0.000007 & 0.001003 & 0.044119 & 0.281517 & $0.5 \pm 0.2$ & 2.30 & 0.281504 & 0.281784 \\
\hline ISK1-04 & 0.281583 & 0.000015 & 0.001780 & 0.074750 & 0.281515 & $0.4 \pm 0.5$ & 2.31 & 0.281504 & 0.281784 \\
\hline ISK1-05 & 0.281593 & 0.000010 & 0.001353 & 0.058850 & 0.281541 & $1.3 \pm 0.3$ & 2.27 & 0.281504 & 0.281784 \\
\hline ISK1-06 & 0.281835 & 0.000038 & 0.003580 & 0.173185 & 0.281698 & $6.9 \pm 1.3$ & 2.06 & 0.281504 & 0.281784 \\
\hline ISK1-07 & 0.281662 & 0.000012 & 0.001584 & 0.074269 & 0.281602 & $3.5 \pm 0.4$ & 2.19 & 504 & 0.281784 \\
\hline ISK1-08 & 0.281634 & 0.000012 & 0.001083 & 0.039637 & 0.281593 & $3.1 \pm 0.4$ & 2.20 & 0.281504 & 0.281784 \\
\hline \multicolumn{3}{|c|}{ Median value } & & & & $2.0 \pm 0.5$ & 2.24 & & \\
\hline KN1-01 & 0.280800 & 0.000020 & 0.000689 & 0.035820 & 0.280772 & $-23.8 \pm 0.7$ & 3.26 & 0.281441 & 0.281712 \\
\hline MK3-01 & 0.281439 & 0.000010 & 0.000640 & 0.028258 & 0.281413 & $-0.9 \pm 0.4$ & 2.43 & 0.281437 & 0.281707 \\
\hline MK3-02 & 0.281476 & 0.000011 & 0.001031 & 0.047157 & 0.281435 & $-0.1 \pm 0.4$ & 2.40 & 0.281437 & 0.281707 \\
\hline MK3-03 & 0.281566 & 0.000015 & 0.001436 & 0.060529 & 0.281508 & $2.5 \pm 0.5$ & 2.31 & 0.281437 & 0.281707 \\
\hline MK3-04 & 0.281525 & 0.000013 & 0.001304 & 0.057755 & 0.281473 & $1.2 \pm 0.5$ & 2.36 & 0.281437 & 0.281707 \\
\hline MK3-05 & 0.281456 & 0.000008 & 0.000818 & 0.037361 & 0.281423 & $-0.5 \pm 0.3$ & 2.42 & 0.281437 & 0.281707 \\
\hline MK3-06 & 0.281420 & 0.000010 & 0.000826 & 0.037954 & 0.281387 & $-1.8 \pm 0.3$ & 2.46 & 0.281437 & 0.281707 \\
\hline MK3-07 & 0.281527 & 0.000011 & 0.001244 & 0.056279 & 0.281477 & $1.4 \pm 0.4$ & 2.35 & 0.281437 & 0.281707 \\
\hline MK3-08 & 0.281468 & 0.000014 & 0.000769 & 0.034297 & 0.281437 & $-0.0 \pm 0.5$ & 2.40 & 0.281437 & 0.281707 \\
\hline \multicolumn{3}{|c|}{ Median value } & & & & $-0.1 \pm 0.4$ & 2.40 & & \\
\hline
\end{tabular}

assign a crystallization age for sample KMP1, we conclude that the protolith to the Kapiri Mposhi granite gneiss component of the Mkushi Gneiss crystallized at ca. $2723 \pm 24 \mathrm{Ma}$.

Sample MK3-Five analyses were conducted on four grains (fig. 3B, table 2). Three analyses were located on rims (2r, 3r and 4r), one analysis was conducted on a single-domain zircon (1) and one analysis sampled a zircon core (4c). Rim analyses $2 \mathrm{r}$ and $3 \mathrm{r}$ recorded low $\mathrm{Th} / \mathrm{U}$ ratios $(0.08$ and 0.10 respectively), suggestive of a metamorphic origin (Hoskin and Black, 2000) and have the youngest ${ }^{207} \mathrm{~Pb} /{ }^{206} \mathrm{~Pb}$ ages of $2015 \pm 13$ and $2031 \pm 10 \mathrm{Ma}$. Analysis pair $4 \mathrm{r}-4 \mathrm{c}$ yielded ${ }^{207} \mathrm{~Pb} /{ }^{206} \mathrm{~Pb}$ crystallization ages of $2044 \pm 23$ and $2037 \pm 15$ Ma respectively. All five data points yield a weighted mean ${ }^{207} \mathrm{~Pb} /{ }^{206} \mathrm{~Pb}$ age of $2032 \pm 15 \mathrm{Ma}(\mathrm{MSWD}=2.8)$. The relatively high MSWD indicates that these data indeed comprise multiple populations. Excluding the two low $\mathrm{Th} / \mathrm{U}$ rim analyses, on the basis that they represent slightly younger zircon growth, and the most discordant data point, a weighted mean ${ }^{207} \mathrm{~Pb} /{ }^{206} \mathrm{~Pb}$ age of $2047 \pm 14 \mathrm{Ma}$ $(\mathrm{MSWD}=0.15)$ can be calculated, which provides the best estimate for the crystallization of the precursor of this component of the Mkushi Gneiss, and is within error of the age of $2049 \pm 6$ Ma reported by Rainaud and others (2005). The slightly younger low $\mathrm{Th} / \mathrm{U}$ rims indicate that this emplacement was directly followed by additional zircon growth between $2031 \pm 10$ and $2015 \pm 13 \mathrm{Ma}$, perhaps linked to the intrusion of aplites, one of which dated by Rainaud and others (2005) at $2036 \pm 22$ Ma. Eight zircon grains were analyzed for $\mathrm{Lu} / \mathrm{Hf}$ isotopic composition, and gave $\varepsilon_{\mathrm{Hf}}(\mathrm{t})$ values between -1.8 and 2.5 and $\mathrm{T}_{\mathrm{DM}}$ model ages between 2.46 and $2.31 \mathrm{Ga}$ indicating a crustal residence age of 300 to 400 Million years (table 3, fig. 4). This is in contrast with the whole rock $\mathrm{Sm}-\mathrm{Nd}$ data reported for the same sample, which gave a $\varepsilon_{\mathrm{Nd}}(\mathrm{t})$ value of -9.2 and a $\mathrm{T}_{\mathrm{DM}}$ model age of $3.25 \mathrm{Ga}$, indicating a much longer crustal residence (De Waele and others, 2006b).

Sample MK5-Seven analyses were conducted on oscillatory zoned domains of seven zircon grains (fig. 3C, table 2). The data show relatively high $\mathrm{U}+\mathrm{Th}$ content and typical magmatic $\mathrm{Th} / \mathrm{U}$ ratios between 0.5 and 0.9 . Five data points loosely define an 


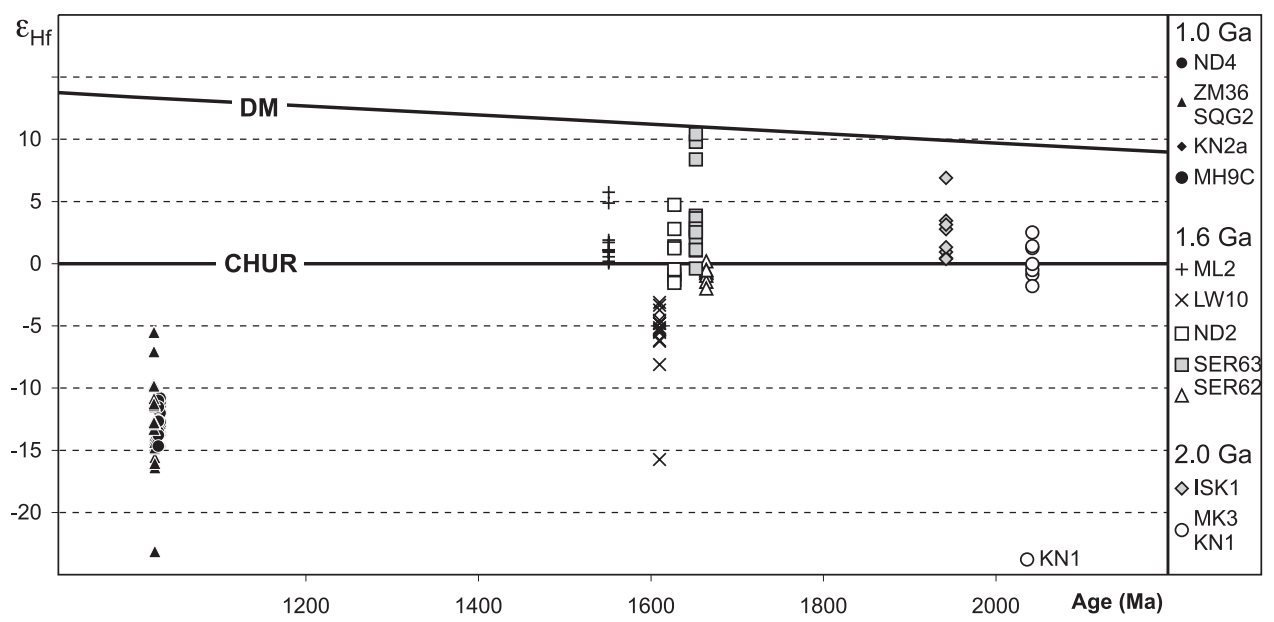

Fig. 4. $\varepsilon_{\mathrm{Hf}}(\mathrm{t})$ versus age plot of data obtained on granitoid rocks in the Irumide Belt.

age group with a weighted mean ${ }^{207} \mathrm{~Pb} /{ }^{206} \mathrm{~Pb}$ age of $2038 \pm 14 \mathrm{Ma}(\mathrm{MSWD}=3.0$ ), while the remaining data points ( 3 and 4 ) yield significantly younger discordant ${ }^{207} \mathrm{~Pb} /{ }^{206} \mathrm{~Pb}$ ages of $2026 \pm 18$ and $1973 \pm 14 \mathrm{Ma}$. The age of $2038 \pm 14 \mathrm{Ma}$ can be adopted for the crystallization age of the granite protolith of this part of the Mkushi Gneiss, again within error of the ages reported by Rainaud and others (2005).

Sample CC10 - Six zircon grains were analyzed and record relatively high $\mathrm{U}+\mathrm{Th}$ content (300-1300 ppm) and Th/U ratios between 0.6 and 0.9 (table 2). The data yielded a weighted mean ${ }^{207} \mathrm{~Pb} /{ }^{206} \mathrm{~Pb}$ age of $1953 \pm 6 \mathrm{Ma}(\mathrm{MSWD}=2.19$, fig. 3D). A Concordia age of $1952 \pm 6 \mathrm{Ma}(\mathrm{MSWD}=1.8)$ can be calculated from the five most concordant points, and is considered the best estimate for the crystallization age of the granite precursor to this sample.

Sample KN1-Six analyses were conducted on six zircon and gave U+Th contents between 330 and $650 \mathrm{ppm}$, with $\mathrm{Th} / \mathrm{U}$ ratios in the range 0.3 to 0.6 (fig. $5 \mathrm{~A}$, table 2). The five most concordant analyses yield a weighted mean ${ }^{207} \mathrm{~Pb} /{ }^{206} \mathrm{~Pb}$ age of $2036 \pm 6$ Ma $(M S W D=1.0)$. The discordant analysis number 1 which yielded an age of $2089 \mathrm{Ma}$ and may be a xenocryst, is excluded from the calculation. Using only the three most concordant data, a Concordia age of $2041 \pm 10 \mathrm{Ma}(\mathrm{MSWD}=0.83)$ can be calculated, which provides the best estimate for the crystallization age of the granite protolith. One zircon was analyzed for $\mathrm{Lu}-\mathrm{Hf}$ isotopic composition (table 3, fig. 4), and recorded an $\varepsilon_{\mathrm{Hf}}(\mathrm{t})$ value of -23.8 and $\mathrm{T}_{\mathrm{DM}}$ of $3.26 \mathrm{Ga}$, possibly indicating that the granite represents a crustal melt. Additional work is needed to confirm the strongly reworked nature of this granite gneiss.

Luwalizi Granite (samples ISK1 and ISK2).- The Luwalizi Granite is a large body of weakly foliated coarse-grained biotite granite that extends from Chinsali (c on fig. 1B) northwards into the Bangweulu Block. It forms the basement to the Manshya River Group, and is correlated with the widespread granitoids of the Bangweulu Block of northern Zambia. Two samples were collected (ISK1 and ISK2 on fig. 1B), and both consist of coarse-grained foliated biotite granite.

Sample ISK1-Nine analyses were conducted on nine different zircon grains (fig. $5 \mathrm{~B}$, table 2). The data show very variable $\mathrm{U}$ and Th content, in the ranges 93 to 2669 $\mathrm{ppm}$ and 109 to $595 \mathrm{ppm}$ respectively, giving variable $\mathrm{Th} / \mathrm{U}$ ratios between 0.04 and 1.93 (table 2). Three analyses, 2, 3 and 6, are excluded from calculations. Analysis 2 

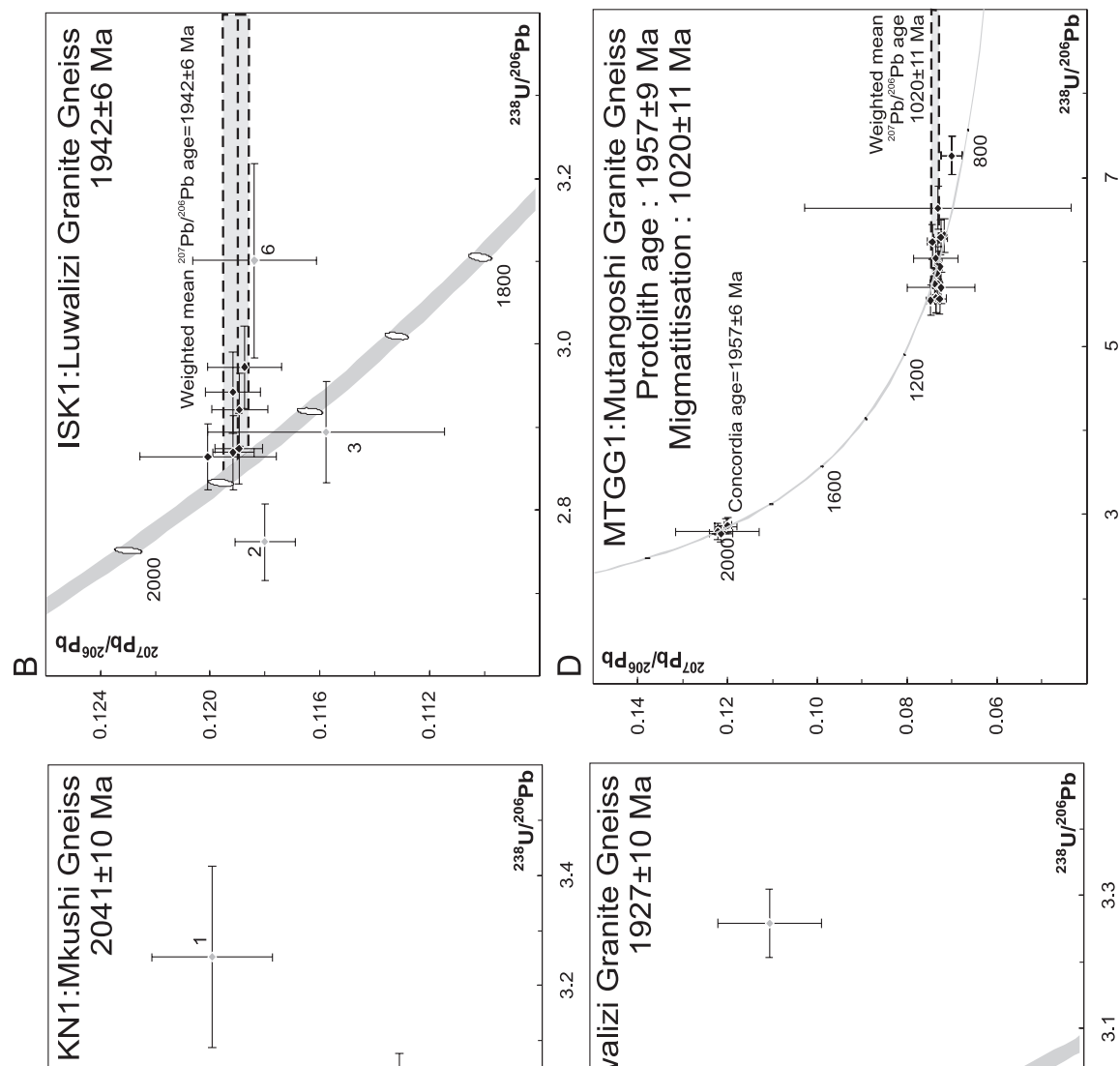

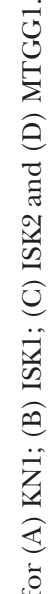

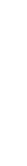

 
displays an unusually high concentration of $U$ (2,669 ppm), whereas analysis 3 yielded high $f_{206}(0.855 \%)$, low $\mathrm{U}(93 \mathrm{ppm})$ and high ${ }^{232} \mathrm{Th} /{ }^{238} \mathrm{U}$ (1.93). Analysis 6 is discordant and records high $\mathrm{U}$ content $(860 \mathrm{ppm})$ and some $\mathrm{Pb}_{\mathrm{c}}$. The remaining analyses plot close to Concordia and define a weighted mean ${ }^{207} \mathrm{~Pb} /{ }^{206} \mathrm{~Pb}$ age of $1942 \pm 6 \mathrm{Ma}(\mathrm{MSWD}=0.23)$, taken to be the best estimate for the crystallization age of the granite in sample ISK1. Eight zircon were analyzed for Lu-Hf isotopic composition, and recorded $\varepsilon_{\mathrm{Hf}}(\mathrm{t})$ values between 0.4 and 6.9 and $\mathrm{T}_{\mathrm{DM}}$ in the range 2.31 to $2.06 \mathrm{Ga}$ indicating some crustal residence (table 3, fig. 4).

Sample ISK2-Five analyses were conducted on five zircon grains (table 2, fig. $5 \mathrm{C})$. The data show the same variable $\mathrm{U}$ and $\mathrm{Th}$ ratios, resulting in a wide range of $\mathrm{Th} / \mathrm{U}$ between 0.03 and 1.34 (table 2). Four of the analyses group in a tight cluster close to Concordia and yield a Concordia age of $1927 \pm 10 \mathrm{Ma}(\mathrm{MSWD}=0.92)$, which we take as the best estimate for the crystallization age of the granite in sample ISK2. Note that the ages for samples ISK1 and ISK2 overlap within uncertainty.

Mutangoshi Granite Gneiss.-The Mutangoshi Granite Gneiss forms a body of strongly foliated to migmatitic biotite granite gneiss in the northeastern Irumide Belt. On the basis of Rb-Sr whole-rock analyses Daly (ms, 1986) determined an emplacement age of $1407 \pm 33 \mathrm{Ma}$ for this body. We collected one sample from a flat outcrop of migmatitic biotite granite gneiss mapped as Mutangoshi Gneissic Granite, near the contact with a younger, undeformed Irumide-age $(\sim 1.0 \mathrm{Ga})$ granitoid (Chilubanama Granite of Daly, ms, 1986). The migmatite is truncated by numerous quartz-rich melts related to the nearby Chilubanama Granite.

Sample MTGG 1-A total of 23 analyses were conducted on 17 different zircon grains (table 2, fig. 5D). Three sets of analyses were conducted on core-rim pairs $(3,6$ and 7 ) one of which yielded significantly different ages. Two analyses were conducted on zircon core. Several analyses on rims and cores were aborted due to high ${ }^{204} \mathrm{~Pb}$ counts and are not included in the data table. Analysis on core $3 \mathrm{c}$ yielded a ${ }^{207} \mathrm{~Pb} /{ }^{206} \mathrm{~Pb}$ age of $1961 \pm 37 \mathrm{Ma}\left(98.6 \%\right.$ concordant), whereas the rim $(3 \mathrm{r})$ gave a ${ }^{207} \mathrm{~Pb} /{ }^{206} \mathrm{~Pb}$ age of $1008 \pm 15 \mathrm{Ma}(99.7 \%$ concordant). The age of the core compares well with analyses $12,16 \mathrm{c}$ and $25 \mathrm{c}$, which yielded concordant ${ }^{207} \mathrm{~Pb} /{ }^{206} \mathrm{~Pb}$ ages of $1955 \pm 12,1989 \pm 136$ and $1978 \pm 38$ Ma respectively. Taken together these four analyses yield a Concordia age of $1957 \pm 6 \mathrm{Ma}$, which is considered the best estimate for the crystallization age of the precursor granite to the Mutangoshi Gneissic Granite. The remaining analyses, including two core-rim pairs (6 and 7) and one analysis on a core (4), define a cluster on the U-Pb evolution diagram (fig. 5D) defining a weighted mean ${ }^{207} \mathrm{~Pb} /{ }^{206} \mathrm{~Pb}$ age of $1020 \pm 11 \mathrm{Ma}(\mathrm{MSWD}=4.6)$. This age corresponds closely to the reported age of the Chilubanama Granite adjacent to the Mutangoshi Gneissic Granite (De Waele and others, 2003; De Waele, ms, 2005), and we interpret these zircons to have crystallized either from granitic melt of the Chilubanama Granite, or from in situ melts related to its intrusion.

POST-MUVA SUPERGROUP AND PRE-IRUMIDE GRANITOIDS AND GNEISSES IN THE IRUMIDE BELT

A suite of granitoid rocks has been recognized which postdates the Paleoproterozoic basement, but predates Irumide tectonism $(1.02 \mathrm{Ga})$ and the voluminous syn- to late- and post-Irumide granitoids (1.05-0.95 Ga). These plutons are known as the Lukamfwa Hill Granite Gneiss in the southwest and as the Lubu Granite Gneiss and Musalango Gneiss in the northeast (LHG, LGG and MSG on fig. 1B). The Lubu Granite Gneiss, was previously believed to be Paleoproterozoic (Daly, ms, 1986), because of its similar character to the Luwalizi Granite Gneiss, which can be traced into granitoids of the Bangweulu Block. The Musalango Gneiss was considered equivalent to the Mutangoshi Gneissic Granite, which was dated using the whole-rock Rb-Sr isochron method at $1407 \pm 33 \mathrm{Ma}$ (Daly, ms, 1986). During this study four samples of 
the Lukamfwa Hill Granite Gneiss (SER62, SER63, SR12 and ND2,), and one each of the Lubu Granite Gneiss (ML2,) and Musalango Gneiss (LW10, fig. 1B) were dated.

\section{The Lukamfwa Hill Granite Gneiss}

The Lukamfwa Granite Gneiss comprises leuco- to mesocratic biotite granite gneiss, with a strong foliation defined by aligned biotite and stretched feldspars. The unit exhibits proto-mylonitic fabrics in local shear zones and commonly contains two deformation fabrics. Samples SER62 and SER63 were collected from Lukamfwa Hill in the Serenje 1:100,000 scale map sheet, whereas SR12 and ND2 were collected from similar granitoid plutons west of Lukamfwa Hill.

Sample SER62-A total of 10 analyses were conducted on eight separate zircon grains (fig. 6A, table 2). $\mathrm{U}$ and Th contents were found to be variable, within the range 118 to $6101 \mathrm{ppm}$, with $\mathrm{Th} / \mathrm{U}$ ratios between 0.38 and 1.16. A high $\mathrm{U}$ and $\mathrm{Pb}_{\mathrm{c}}$ content for 41c led us to exclude this data point from age calculations. Using the seven most concordant points, a Concordia age of $1665 \pm 4$ Ma with MSWD of 0.14 is obtained, which we take as the best estimate for the crystallization age of this sample. Eight zircon grains were analyzed for $\mathrm{Lu}-\mathrm{Hf}$ isotopic composition, and recorded $\varepsilon_{\mathrm{Hf}}(\mathrm{t})$ values between -2.0 and 0.2 and a narrow range of $\mathrm{T}_{\mathrm{DM}}, 2.15$ to $2.07 \mathrm{Ga}$, slightly younger than the model age range for the Mkushi Gneiss (table 3, fig. 4). These data suggest that the Lukamfwa Granite Gneiss was largely derived from reworked crust, but received substantial juvenile input as well.

Sample SER63-Ten analyses were conducted on oscillatory zoned sectors of ten zircon grains (table 2, fig. 6B). U and Th contents were a lot more consistent, with ranges of 280 to $470 \mathrm{ppm}$ and 211 to $356 \mathrm{ppm}$ respectively, and a narrow range of $\mathrm{Th} / \mathrm{U}$ ratios between 0.59 and 1.11. A Concordia age of $1650 \pm 8 \mathrm{Ma}$ can be calculated for the analyses $(\mathrm{MSWD}=1.40)$, which we consider to be the best age estimate for the crystallization of the precursor granite in sample SER 6-3. Lu-Hf isotopic composition was obtained for twelve grains, and gave $\varepsilon_{\mathrm{Hf}}(\mathrm{t})$ values between -0.4 and 10.4 and a range of $\mathrm{T}_{\mathrm{DM}} 1.67$ to $2.08 \mathrm{Ga}$ indicating more juvenile input for this sample than for sample SR62 (table 3, fig. 4). Whole rock Sm-Nd data reported for a sample collected from the same locality (SR7, see De Waele and others, $2006 \mathrm{~b})$, yielded an $\varepsilon_{\mathrm{Nd}}(\mathrm{t})$ value of -10.0 and a $\mathrm{T}_{\mathrm{DM}}$ model age of $3.23 \mathrm{Ga}$, indicating significant crustal residence. It is unclear whether these data reflect a composite nature of the Lukamfwa Granite, with locally unmixed portions of juvenile magma within a dominantly reworked melt.

Sample SR12-Seven analyses were conducted on seven different zircon grains (table 2, fig. 6C). $\mathrm{U}+\mathrm{Th}$ values range from 215 to $478 \mathrm{ppm}$, with $\mathrm{Th} / \mathrm{U}$ ratios between 0.63 and 1.19. All data are near concordant and define $\mathrm{a}{ }^{207} \mathrm{~Pb} /{ }^{206} \mathrm{~Pb}$ age of $1639 \pm 14$ Ma $(M S W D=0.91)$. However, a slight drift off Concordia could represent Pb-loss at $1020 \mathrm{Ma}$, the age of metamorphism in the Irumide Belt (De Waele and others, 2006a), and a regression towards that gives an upper intercept at $1638 \pm 30 \mathrm{Ma}$ (MSWD = 0.04 ). The four most concordant data points correspond to a Concordia age of $1655 \pm$ $11 \mathrm{Ma}(\mathrm{MSWD}=1.80)$, which we interpret as the best estimate for the crystallization age of this granite. Whole rock Sm-Nd data reported in De Waele and others (2006b), yielded an $\varepsilon_{\mathrm{Nd}}(\mathrm{t})$ value of -8.8 and $\mathrm{T}_{\mathrm{DM}}$ model age of $2.85 \mathrm{Ga}$, reflecting an origin by melting of Archean crust.

Sample ND2-Four analyses were conducted on four zircon grains (table 2, fig. 6D) and gave a narrow spread of $\mathrm{U}$ and Th values, 103 to $229 \mathrm{ppm}$ and 82 to $196 \mathrm{ppm}$ respectively, giving $\mathrm{Th} / \mathrm{U}$ ratios between 0.83 and 0.90 . The data are near concordant, and define an age group with Concordia age of $1627 \pm 12 \mathrm{Ma}(\mathrm{MSWD}=0.51)$, which is taken to represent the crystallization age of the granite protolith. Eight zircons were analyzed for Lu-Hf isotopic composition, and recorded $\varepsilon_{\mathrm{Hf}}(\mathrm{t})$ values between -1.5 and 


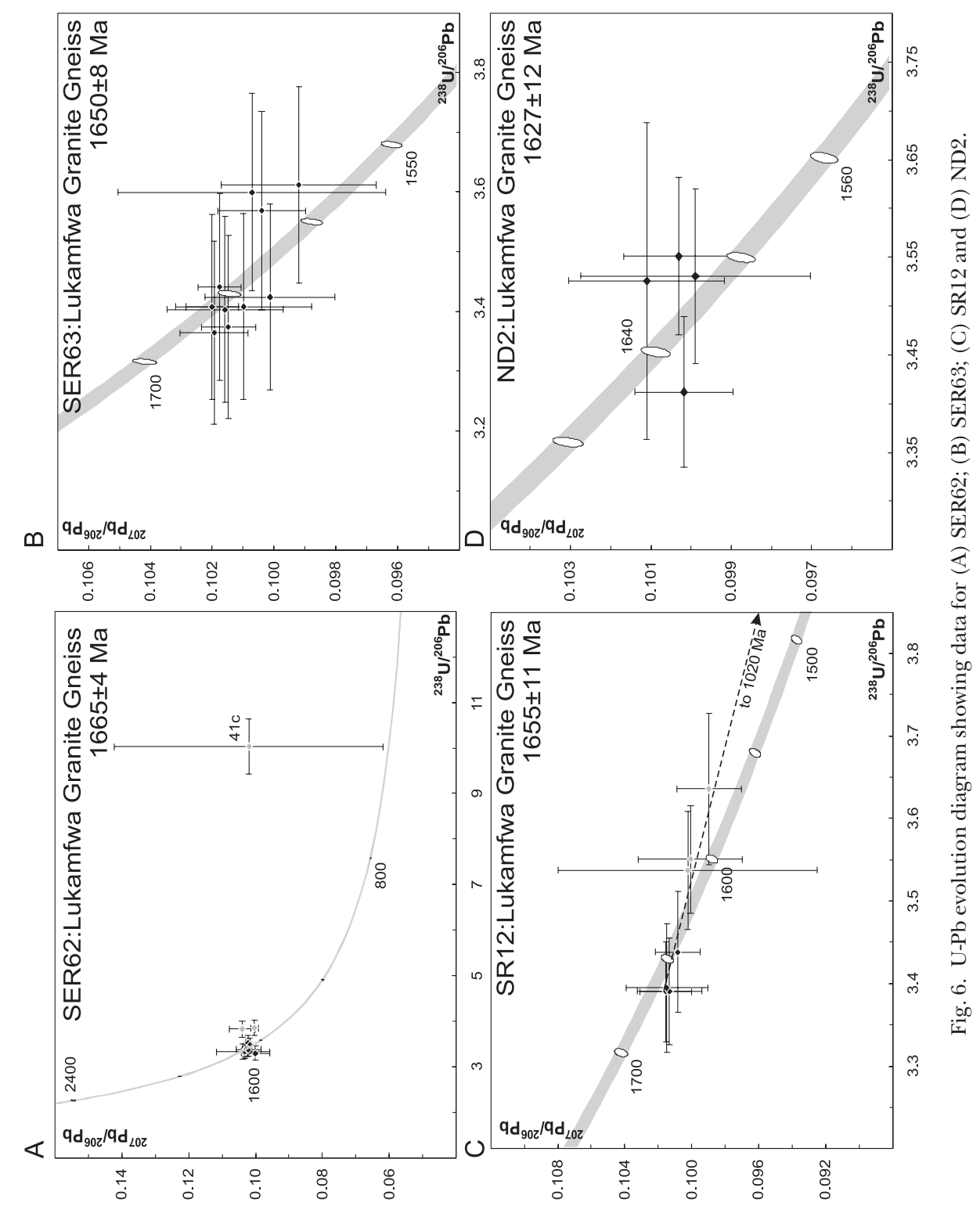


4.7 and a range of $\mathrm{T}_{\mathrm{DM}} 2.12$ to $1.86 \mathrm{Ga}$, indicating a mix of crustal and juvenile melt in the sample (table 3 , fig. 4 ).

\section{The Lubu Granite Gneiss}

Sample ML2-Sample ML2 was collected from the Lubu Granite Gneiss (marked LGG, fig. 1B), which was interpreted by Daly (ms 1986, 1995a) to form the basement to the Muva Supergroup in the northeastern Irumide Belt. The Lubu Granite Gneiss is an equigranular, strongly foliated pink biotite granite gneiss, which in places is intruded by numerous small tightly folded pegmatites. Eleven clear, uncracked portions of eleven zircon crystals were analyzed during two separate sessions (table 2, fig. 7A). The data are characterized by high $\mathrm{Pb}_{c}$ and unusually high $\mathrm{Th} / \mathrm{U}$ ratios (table 2 ). A regression line through all data points defines an upper intercept of $1547 \pm 30 \mathrm{Ma}$ and lower intercept $465 \pm 440 \mathrm{Ma}(\mathrm{MSWD}=0.76)$. A weighted mean ${ }^{207} \mathrm{~Pb} /{ }^{206} \mathrm{~Pb}$ age of all but the two most discordant points gives an age of $1532 \pm 14 \mathrm{Ma}(\mathrm{MSWD}=0.97)$. We take the latter to represent the best age estimate for crystallization of zircon in the protolith of the granite gneiss. Ten zircon grains were analyzed for $\mathrm{Lu}-\mathrm{Hf}$ isotopic composition, and recorded $\varepsilon_{\mathrm{Hf}}(\mathrm{t})$ values between 0.0 and 5.7 and a range of $\mathrm{T}_{\mathrm{DM}}$ of 1.98 to $1.78 \mathrm{Ga}$, indicating a juvenile character with possibly some limited crustal residence time for the protolith of the Lubu Granite Gneiss (table 3, fig. 4).

\section{The Musalango Gneiss}

Sample LW10-The Musalango Gneiss forms a well-defined pluton in the northeastern Irumide Belt, comprising foliated gray biotite granite (MSG on fig. 1B). It was mapped by Daly (ms 1986, 1995a) as part of the Mutangoshi Gneissic Granite, which had yielded a whole-rock Rb-Sr date of $\sim 1.4 \mathrm{Ga}$. Thirteen analyses were carried out on thirteen grains. The data are characterized by low $\mathrm{U}$ and Th content (see table 2), leading to relatively imprecise ratios. Excluding the five analyses with lowest $\mathrm{U}$ and highest $\mathrm{Pb}_{\mathrm{c}}$, a Concordia age of $1587 \pm 13 \mathrm{Ma}(\mathrm{MSWD}=2.4$, see fig. 7B) can be calculated, which we take to represent the best estimate for the crystallization of zircon in sample LW10. 14 zircons yielded Lu-Hf isotopic compositions from which a range of $\varepsilon_{\mathrm{Hf}}(\mathrm{t})$ values between -3.1 and -15.7 and $\mathrm{T}_{\mathrm{DM}}$ between 2.62 and $2.14 \mathrm{Ga}$ were calculated (table 3, fig. 4). These data clearly indicate a significant crustal component in this sample, with possible derivation from Mesoarchean source material.

\section{LATE-MESOPROTEROZOIC INTRUSIONS: THE IRUMIDE GRANITOIDS}

A total of 25 samples from Irumide granitoid bodies were collected across the Irumide Belt between Kapiri Mposhi and Isoka ( $\mathrm{k}$ and is on fig. 1B, table 2) and include foliated and unfoliated granitoids. Sample KK1 was taken from a flat outcrop along the sealed road near Kapiri Mposhi, and consists of porphyritic biotite granite with elongate fine grained angular xenoliths of mafic material, possibly broken-up mafic dikes. A foliation along northerly direction, dipping steeply to the south, is defined by strongly aligned biotite, wrapping around essentially euhedral large microcline phenocrysts. Sample MH4 was collected from a flat outcrop of foliated porphyritic granite, cut by various aplite dikes, which are folded along axes plunging shallowly to the north. Microcline phenocrysts are euhedral and aligned along an east-west direction, defining a shallow northerly-dipping fabric interpreted as primary magmatic layering. Sample MH9 was taken from a large whaleback outcrop of megacrystic granite, with abundant megacrysts of microcline of up to $5 \mathrm{~cm}$ in length. The granite is cut by numerous shallow-dipping aplitic dikes, which appear to follow a flat-lying primary magmatic fabric defined by aligned megacrysts. A shallow north-dipping foliation along west-southwest direction is defined by alignment of biotite flakes. Sample CC5 was collected from a large flat outcrop of porphyritic granite, intruded by various aplite dikes. The granite also contains small late melt veins, in which abundant 

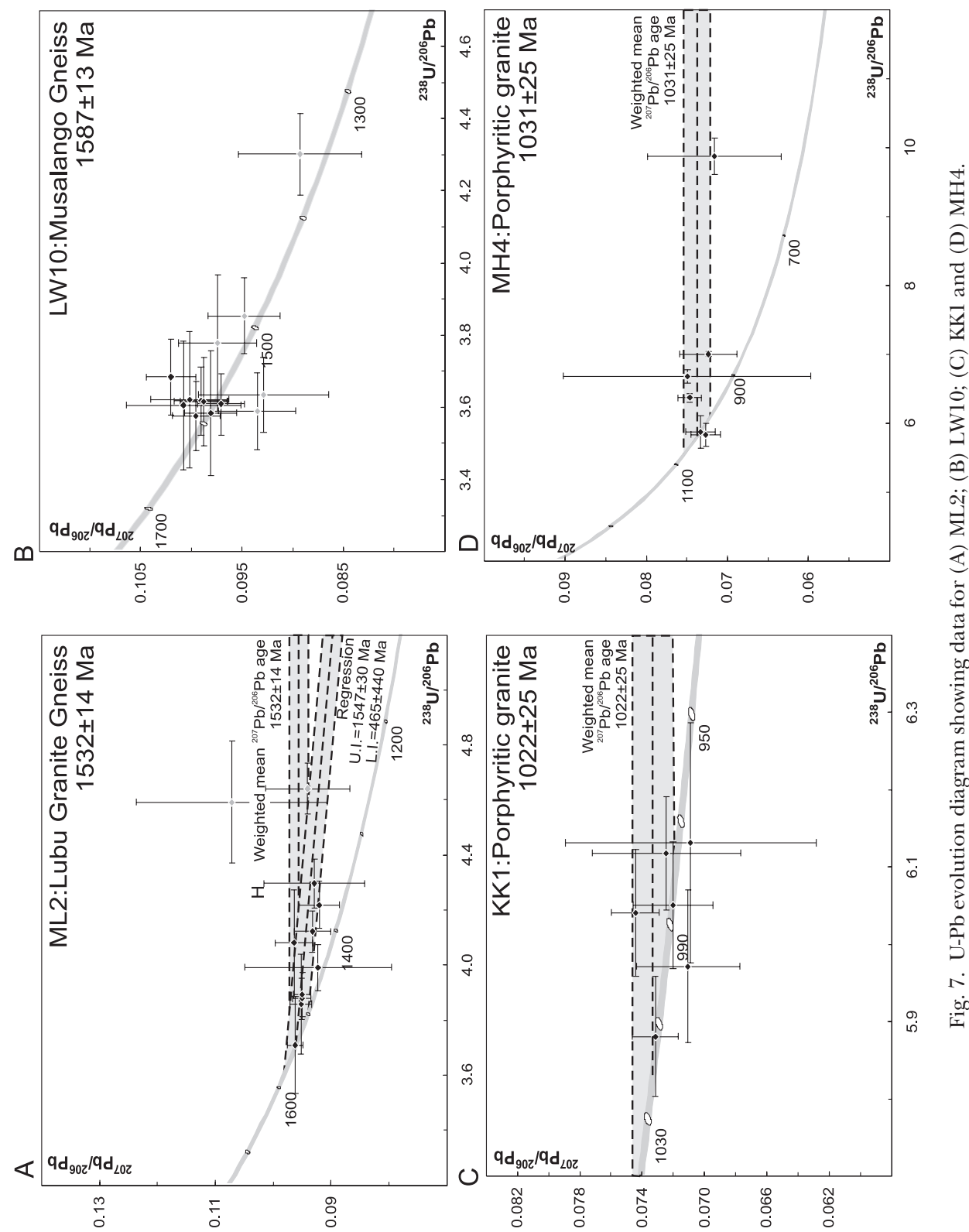
garnet was observed. Sample CC8 was collected from a flat outcrop of foliated porphyritic biotite granite similar to sample CC5. Sample MK7 was collected from foliated reddish biotite granite south of Mkushi ( $\mathrm{mk}$ on fig. 1B). The granite is intruded by various cross-cutting small pegmatites. Both the granite and the pegmatites appear deformed and a series of mafic xenoliths occur along the foliation, which is trending east-northeast, dipping steeply to the east. Sample ND1 consists of coarse porphyritic biotite granite, with a very weak biotite foliation, and large microcline phenocrysts aligned along a north-south oriented magmatic layering. The phenocrysts are euhedral, and do not appear to be affected by the weak foliation. Sample ND4 was collected from a small circular body of granodiorite. The granodiorite is porphyritic, and contains minor quartz, large plagioclase and abundant hornblende in a subophitic texture, and subordinate biotite, defining a weak foliation. Sample ND5 was collected from a body of syeno-granite within the porphyritic granites. Although the contact between the porphyritic granites and the syeno-granite is not exposed, the weak deformation and similarities in fabric suggest the intrusion was emplaced at the same time as the granite. The rock consists of abundant phenocrysts of microcline set in a matrix of plagioclase and microcline, with subordinate quartz and biotite. The syeno-granite is cut by various aplitic dikes, which appear to display the same weak foliation as the host rock, and were emplaced perhaps during late stages of intrusion, and prior to the deformation that imparted the weak fabrics. Sample FW1 was collected from a flat outcrop of coarse porphyritic foliated granite, while sample FW2 came from an aplite dike cutting the granite. The foliation observed in the granite is defined by biotite, while microcline phenocrysts appear undeformed, and are aligned along a magmatic layering. The foliation does not seem to affect the aplite dikes, which consist of fine-grained microcline and quartz. Sample SASA2 was collected from a dome of leucocratic, mildly foliated granite north of Serenje ( $\mathrm{sr}$ on fig. 1B), while sample SER53 came from a body of porphyritic biotite granite to the south of that. Samples SER64 and SQG2 come from similar K-feldspar porphyritic granitoid bodies south of Serenje. Sample KN2a was sampled from foliated biotite granite with large K-feldspar phenocrysts. Fine-grained aplitic dikes that contain garnet cut the granite gneiss. Sample KN5 was collected from a flat outcrop of foliated porphyritic biotite granite. The granite contains abundant xenoliths of mafic composition, as well as rafts of metasedimentary rocks, migmatitic material and fine-grained granite gneiss. The granite carries a prominent penetrative foliation along a north-south direction, dipping steeply to the west. Samples KN7 and KN8 were collected from two different inselbergs, consisting entirely of foliated two-mica granite. The granite is foliated along northeasterly trends, dipping shallowly to steeply to the northwest. Euhedral Kfeldspar phenocrysts define a north-south trending primary magmatic fabric. Sample CHT6 comes from a flat lying outcrop of coarse, foliated biotite granite, which contains abundant small dark xenoliths of metasedimentary origin. The granite is cut by a biotite foliation oriented northeast and steeply dipping to the southeast. Sample ZM36 was collected from a leucocratic biotite granite in a quarry along the main road halfway between Serenje and Mpika (sr and mp respectively on fig. 1B). Sample CHL5 was taken from a flat outcrop to the southwest of Mpika, where strongly foliated micaceous granite gneiss is intruded by numerous small pegmatites. The gneiss shows a marked biotite-muscovite foliation along northeasterly trends, with steep northdirected dip. The Chilubanama Granite (or Grey Granite) described in detail by Daly (1995a), was sampled from four localities around Chinsali (samples LW1, LW2, MTG4 and ZM32). In all localities, the granite occurs as gray, biotite granite, with minor garnet in the case of sample MTG4. The Chilubanama Granite is commonly unfoliated and in places shows primary igneous fabrics. 
Sample KK1-Six analyses were conducted on six single-domain zircon. U and Th show a narrow spread, with $\mathrm{Th} / \mathrm{U}$ ratios between 0.50 and 1.35 . The data show too much scatter to define a Concordia age, but define a narrow range of ${ }^{207} \mathrm{~Pb} /{ }^{206} \mathrm{~Pb}$ ratios, giving a weighted mean age of $1022 \pm 25 \mathrm{Ma}$ (MSWD $=1.08$; fig. 7C). We consider this age a reasonable estimate for the timing of crystallization.

Sample MH4 - Six zircons were analyzed during two sessions and yield variable $U$ and $\mathrm{Th}$ contents, with $\mathrm{U}+\mathrm{Th}$ in the range 165 to $1862 \mathrm{ppm}$, and $\mathrm{Th} / \mathrm{U}$ ranging between 0.32 and 1.67. The data define a regression towards present-day Pb-loss, which gives an age of $1031 \pm 25 \mathrm{Ma}(\mathrm{MSWD}=0.55$, fig. 7D). We take this to be the best estimate for the crystallization age of the granite.

Sample MH9 - Seven analyses were conducted over two separate sessions (fig. 6A, table 2). $\mathrm{U}$ and Th contents are variable, in the ranges 120 to $808 \mathrm{ppm}$ and 79 to 632 $\mathrm{ppm}$ respectively, but with a narrow range of $\mathrm{Th} / \mathrm{U}$ ratios between 0.68 and 0.84 . The data define a weighted mean ${ }^{207} \mathrm{~Pb} /{ }^{206} \mathrm{~Pb}$ age of $1035 \pm 10 \mathrm{Ma}(\mathrm{MSWD}=0.59$, fig. $8 \mathrm{~A}$ ), which we take as the best estimate of the age of the granite. Eight Lu-Hf isotopic analyses were conducted, which record a narrow range of $\varepsilon_{\mathrm{Hf}}(\mathrm{t})$ values between -11.0 and -14.7 and $\mathrm{T}_{\mathrm{DM}}$ model ages between 2.07 and $1.97 \mathrm{Ga}$, indicating a substantial crustal component in the granite, with crustal residence times of up to a Billion years (table 3, fig. 4).

Sample CC5-Six analyses were conducted on five zircon crystals, including one core-rim pair (fig. 8B, table 2). The data define a narrow range of $\mathrm{U}$ and $\mathrm{Th}$ contents, and $\mathrm{Th} / \mathrm{U}$ ratios between 0.40 and 0.62 . The core-rim pair recorded overlapping ages and both are characterized by concentric zoning patterns indicative of magmatic crystallization. A Concordia age of $1038 \pm 17 \mathrm{Ma}(\mathrm{MSWD}=0.32)$ can be calculated on all data points, and is taken as the best estimate for the crystallization age of the granite.

Sample CC8 - Six analyses were conducted on six clear zircon grains (fig. 8C, table 2). U+Th content ranges from 396 to $800 \mathrm{ppm}$, with $\mathrm{Th} / \mathrm{U}$ ratios between 0.42 and 0.66. One spot (5) shows an elevated $\mathrm{Pb}_{\mathrm{c}}$, and plots slightly away from a concordant cluster for which a Concordia age of $1035 \pm 12 \mathrm{Ma}(\mathrm{MSWD}=0.62)$ can be calculated, representing the best estimate for the age of crystallization of the granite.

Sample MK7-Six analyses were conducted on five zircons, including one corerim pair (2c and $2 \mathrm{r}$, see fig. $8 \mathrm{D}$, table 2 ). A low $\mathrm{Th} / \mathrm{U}$ ratio $(0.04)$ was obtained from rim analysis $2 \mathrm{r}$, which defines a concordant ${ }^{206} \mathrm{~Pb} /{ }^{238} \mathrm{U}$ age of $883 \pm 10 \mathrm{Ma}$ and could reflect a metamorphic and/or metasomatic event. The analysis on the core (2c) yields a discordant data point which defines a ${ }^{207} \mathrm{~Pb} /{ }^{206} \mathrm{~Pb}$ age of $2003 \pm 72 \mathrm{Ma}$ and represents a xenocryst. The remaining four analyses display a range of ${ }^{207} \mathrm{~Pb} /{ }^{206} \mathrm{~Pb}$ isotopic ratios with a weighted mean of $1079 \pm 150$ Ma (MSWD $=2.5$ ). The most concordant data point of this set gives a ${ }^{207} \mathrm{~Pb} /{ }^{206} \mathrm{~Pb}$ age of $1035 \pm 32 \mathrm{Ma}$ which could approximate the emplacement age of the granite. In summary, the data on this sample are insufficient to calculate an unequivocal age, neither for emplacement of the protolith or for the overprinting event(s).

Sample ND1-Ten analyses were conducted on nine zircon grains, including one core-rim pair (5c and 5r, fig. 9A, table 2). Analyses 1, 3, 5r, 7 and 8 yielded highly discordant results and are characterized by large $f_{206}$ values and resulting poor precision (they are therefore now shown on the plot of fig. 9A). Five points do define a Concordia age of $1034 \pm 6 \mathrm{Ma}$ (fig. 9A), which we take as the best estimate for the crystallization age of the granite.

Sample ND4-Four analyses were conducted on four euhedral zircon grains (fig. 9B, table 2). The data are characterized by relatively high $\mathrm{Th} / \mathrm{U}$ ratios, between 1.10 and 1.69. The data plot on Concordia and define a Concordia age of $1031 \pm 9 \mathrm{Ma}$ $($ MSWD $=0.74)$, which we consider the best estimate for crystallization of the granodiorite. Ten zircons were analyzed for $\mathrm{Lu}-\mathrm{Hf}$ isotopic composition and record 

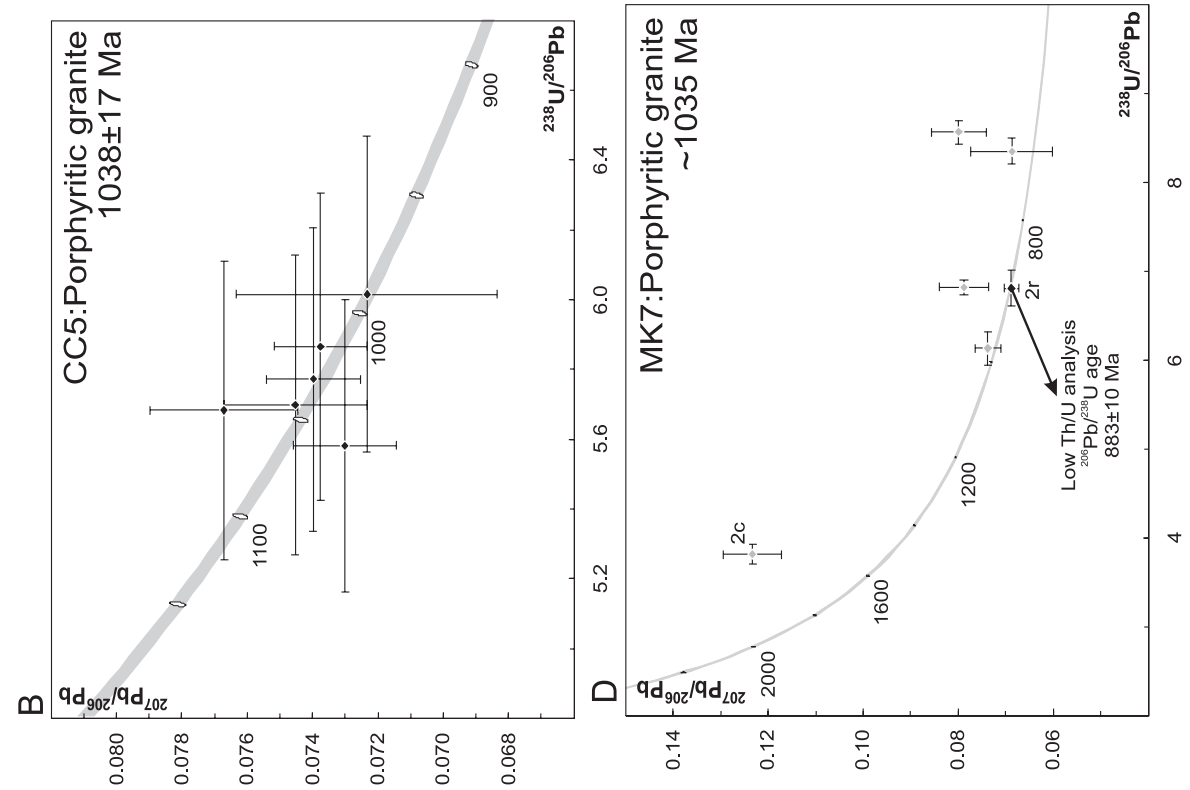

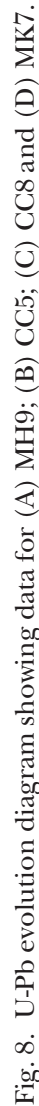
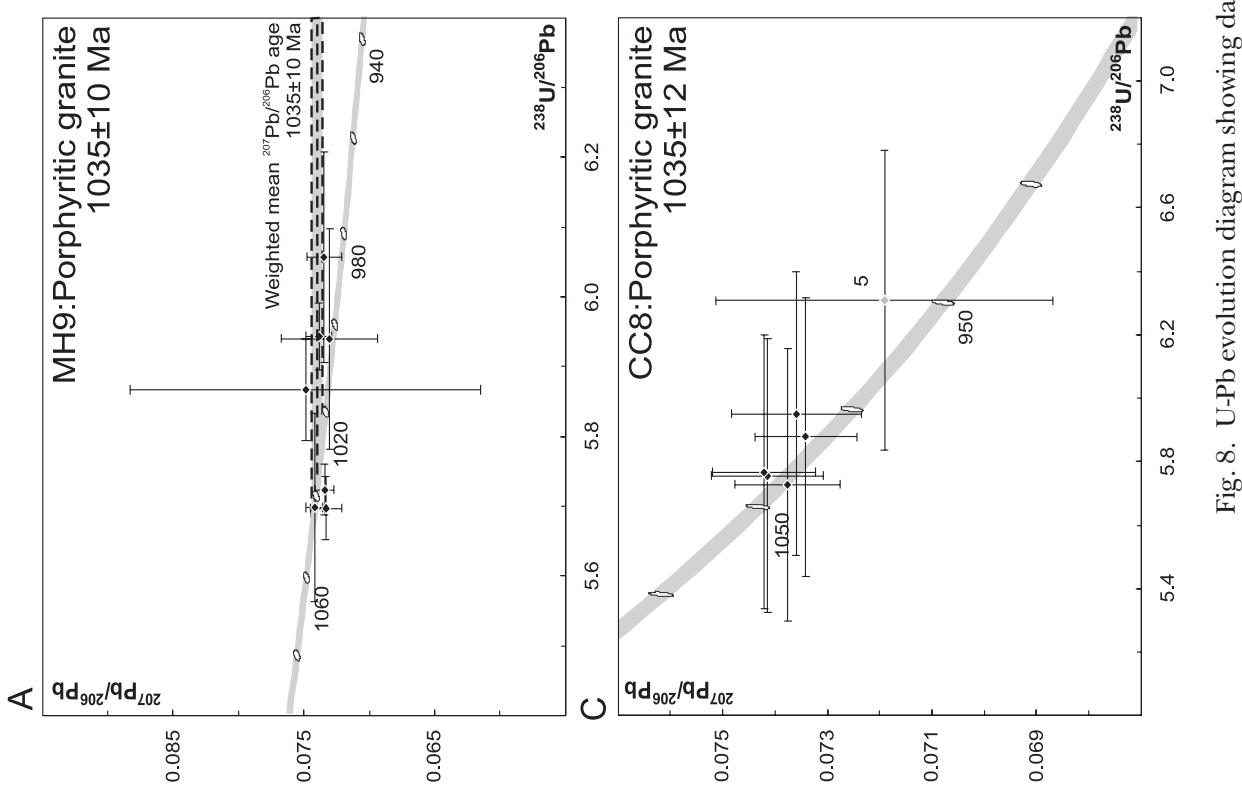

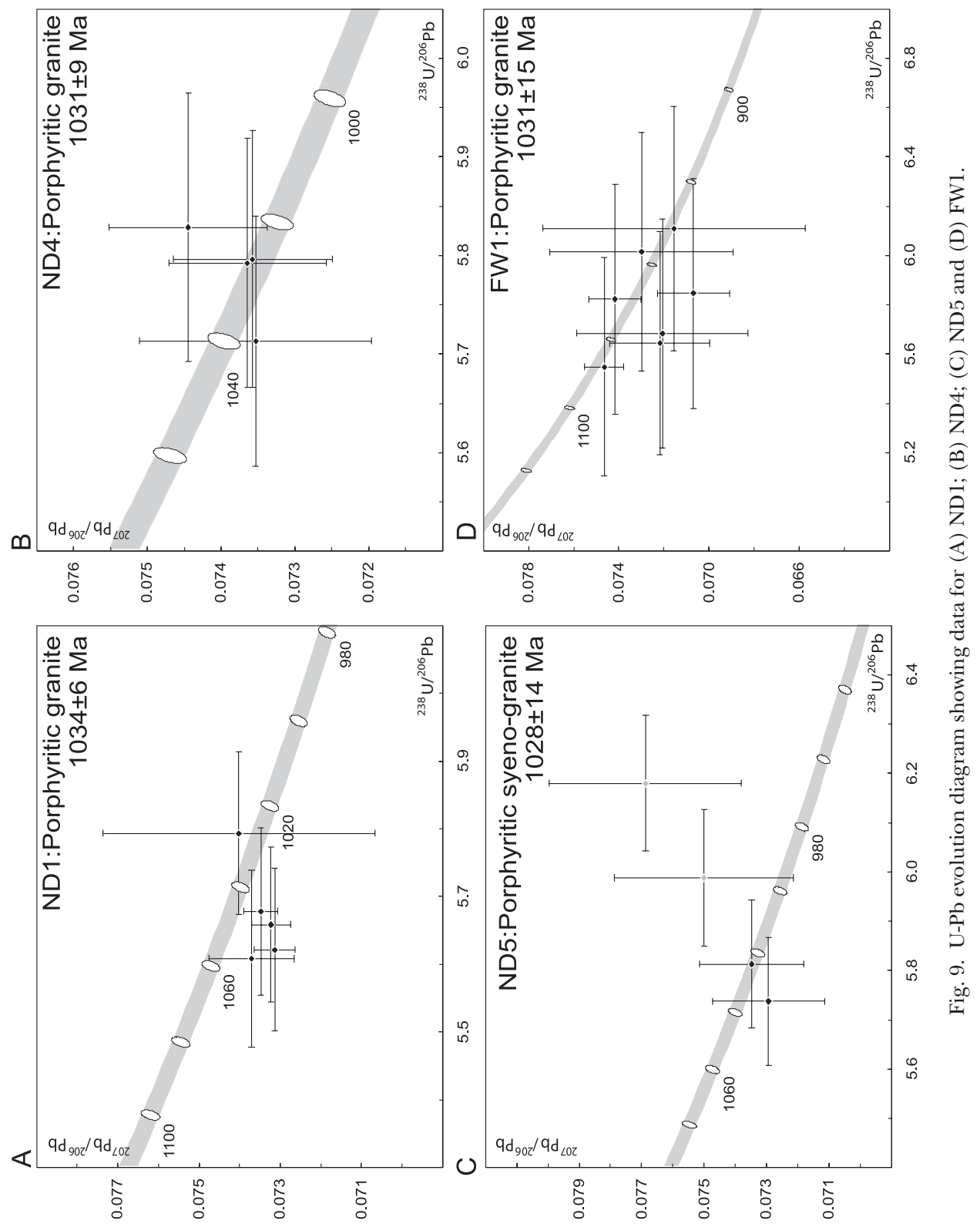
$\varepsilon_{\mathrm{Hf}}(\mathrm{t})$ values between -13.1 and -10.8 and $\mathrm{T}_{\mathrm{DM}}$ model ages between 2.09 and $1.93 \mathrm{Ga}$, indicating a substantial crustal component in the granite (table 3, fig. 4).

Sample ND5-Four zircons were analyzed, but two plot off Concordia due to overestimation of $\mathrm{Pb}_{\mathrm{c}}$ (fig. 9C). The data show a narrow range of $\mathrm{U}$ and $\mathrm{Th}$ contents, as well as $\mathrm{Th} / \mathrm{U}$ ratios. Two concordant points define a Concordia age of $1028 \pm 14 \mathrm{Ma}$ $(\mathrm{MSWD}=0.25)$, which we can take as the best estimate for the age of the syenogranite.

Samples FW1 (granite) and FW2 (aplite dike)-Seven analyses were conducted on seven different zircon grains of the granite. The data indicate highly variable $U$ content, between 203 and 2020 ppm, and variable Th/U ratios between 562 and 2442. The data yield a Concordia age of $1031 \pm 15 \mathrm{Ma}($ fig. $9 \mathrm{D}$; MSWD $=0.15$ ) taken as a good approximation of the crystallization age of the porphyritic granite. Zircon grains from the aplite are subhedral, and comprise a wide variety of types based on morphology (see fig. 2D). $\mathrm{U}$ and Th values are also quite variable, with ranges of 77 to $1375 \mathrm{ppm} \mathrm{U}$ and 22 to $237 \mathrm{ppm}$ Th, leading to Th/U ratios between 0.07 and 2.16. Thirteen analyses were completed on thirteen zircon grains (table 2), and additional analyses were abandoned due to high $\mathrm{Pb}_{\mathrm{c}}$. Nine zircon appear to define a single age group, for which a Concordia age of $2032 \pm 12$ Ma can be calculated (MSWD $=0.51$, fig. 10A). We interpret this age to reflect underlying granitic basement and the zircon to be xenocrystic. Of the remaining five zircon analyses, two are concordant and define ${ }^{207} \mathrm{~Pb} /{ }^{206} \mathrm{~Pb}$ ages of $2773 \pm 46 \mathrm{Ma}$ and $1637 \pm 93 \mathrm{Ma}$ corresponding to additional basement sources sampled by the aplite.

Sample SASA2-A total of 10 zircon grains were analyzed of sample SASA2, including two core-rim pairs (figs. 2D, 10B; table 2 ). $\mathrm{U}$ and Th are very variable, as are the $\mathrm{Th} / \mathrm{U}$ ratios. Analyses of the two core-rim pairs yielded identical results (within error) as analyses on single-growth zircon. A Concordia age can be calculated from the nine most concordant data points, yielding $1023 \pm 13 \mathrm{Ma}(\mathrm{MSWD}=0.13)$, which we take to be the most reliable estimate for the age of the Sasa Granite.

Sample SER53-Ten different zircon grains were analyzed (table 2), three of which show slightly elevated $\mathrm{Pb}_{\mathrm{c}}$ and plot away from Concordia (fig. 10C). U and Th content are in the range 117 to $813 \mathrm{ppm}$ and 123 to $761 \mathrm{ppm}$ respectively, with Th/U between 0.50 and 1.74. A concordant cluster of seven data points corresponds to an age of $1036 \pm 11 \mathrm{Ma}(\mathrm{MSWD}=0.56$, see fig. 10C), which we take as the best age estimate for crystallization of zircon in SER53.

Sample SER64-Ten analyses were conducted on ten zircon grains (fig. 10C, table 2). One analysis yielded a concordant ${ }^{207} \mathrm{~Pb} /{ }^{206} \mathrm{~Pb}$ age of $2000 \pm 13 \mathrm{Ma}$ and is interpreted as a xenocryst. Excluding one inversely discordant data point and two with high $\mathrm{Pb}_{\mathrm{c}}$ a Concordia age of $1037 \pm 11 \mathrm{Ma}(\mathrm{MSWD}=1.12)$ can be calculated, which we consider the best estimate for the crystallization age of the porphyritic granite. It is worth noting that whole rock Sm-Nd analysis of a sample from the same locality, sample SR5 reported in De Waele and others (2006b), yielded an $\varepsilon_{\mathrm{Nd}}(\mathrm{t})$ value of -15.2 and $\mathrm{T}_{\mathrm{DM}}$ model age of $2.63 \mathrm{Ga}$, indicating this granite to be a crustal melt.

Sample SQG2_-Eleven analyses were conducted on eleven zircon grains. U+Th contents are quite high, between 275 and $1048 \mathrm{ppm}$, with Th/U ratios between 0.38 and 1.04 (table 2). The data define a broad cluster close to Concordia with a weighted mean ${ }^{207} \mathrm{~Pb} /{ }^{206} \mathrm{~Pb}$ age of $1032 \pm 16 \mathrm{Ma}(\mathrm{MSWD}=3.4$, fig. 11A). Using only a cluster of six coherent and concordant data points $(7,17,20,41,48$ and 60), a Concordia age of $1020 \pm 8 \mathrm{Ma}(\mathrm{MSWD}=2.8)$ can be calculated, which we propose to be the best estimate for the crystallization age of the granite. Twelve Lu-Hf isotopic analyses were conducted, recording a range of $\varepsilon_{\mathrm{Hf}}(\mathrm{t})$ values between -13.3 and -5.5 and $\mathrm{T}_{\mathrm{DM}}$ model ages between 2.03 and $1.79 \mathrm{Ga}$, indicating the crustally reworked nature of the granite (table 3, fig. 4). 

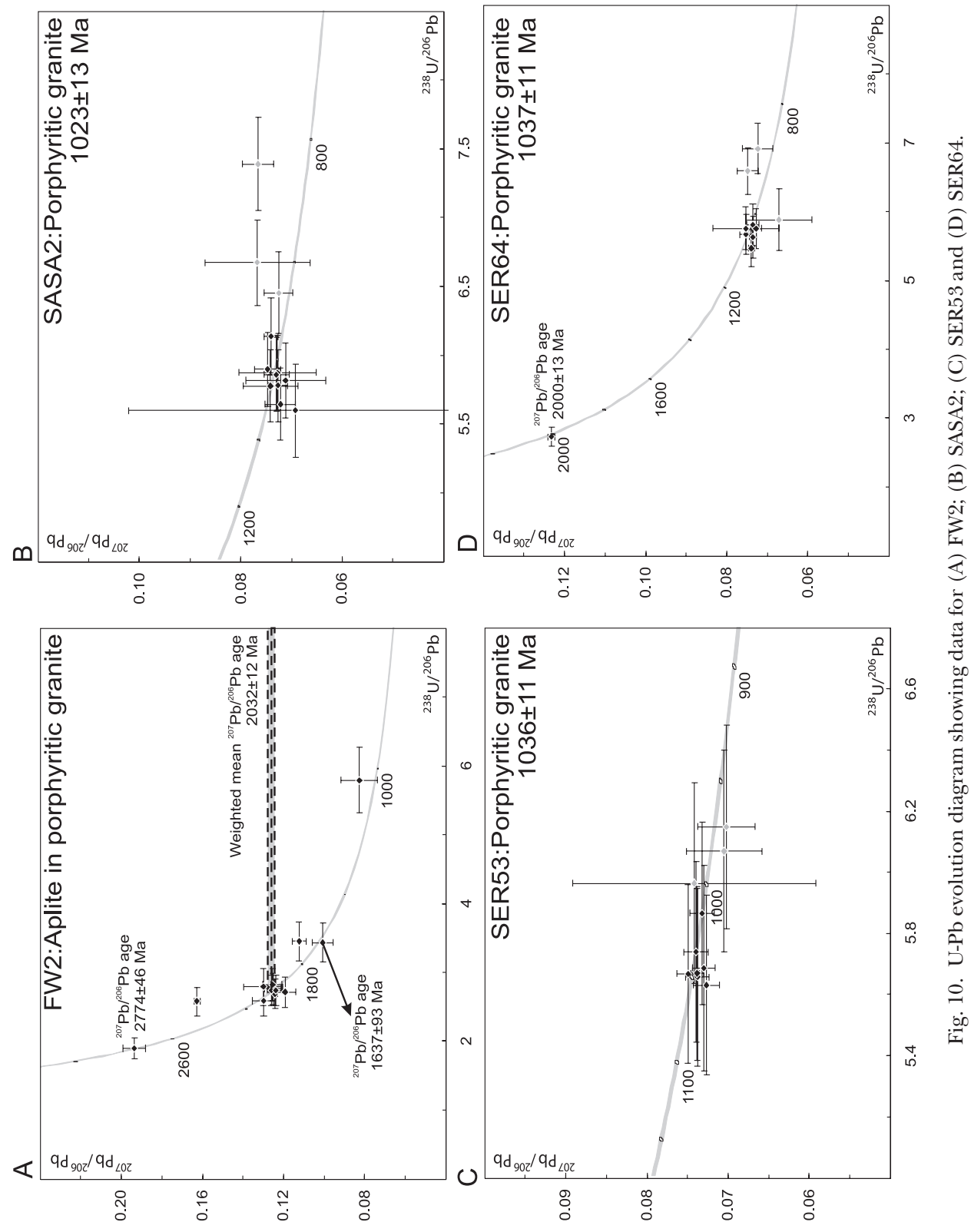

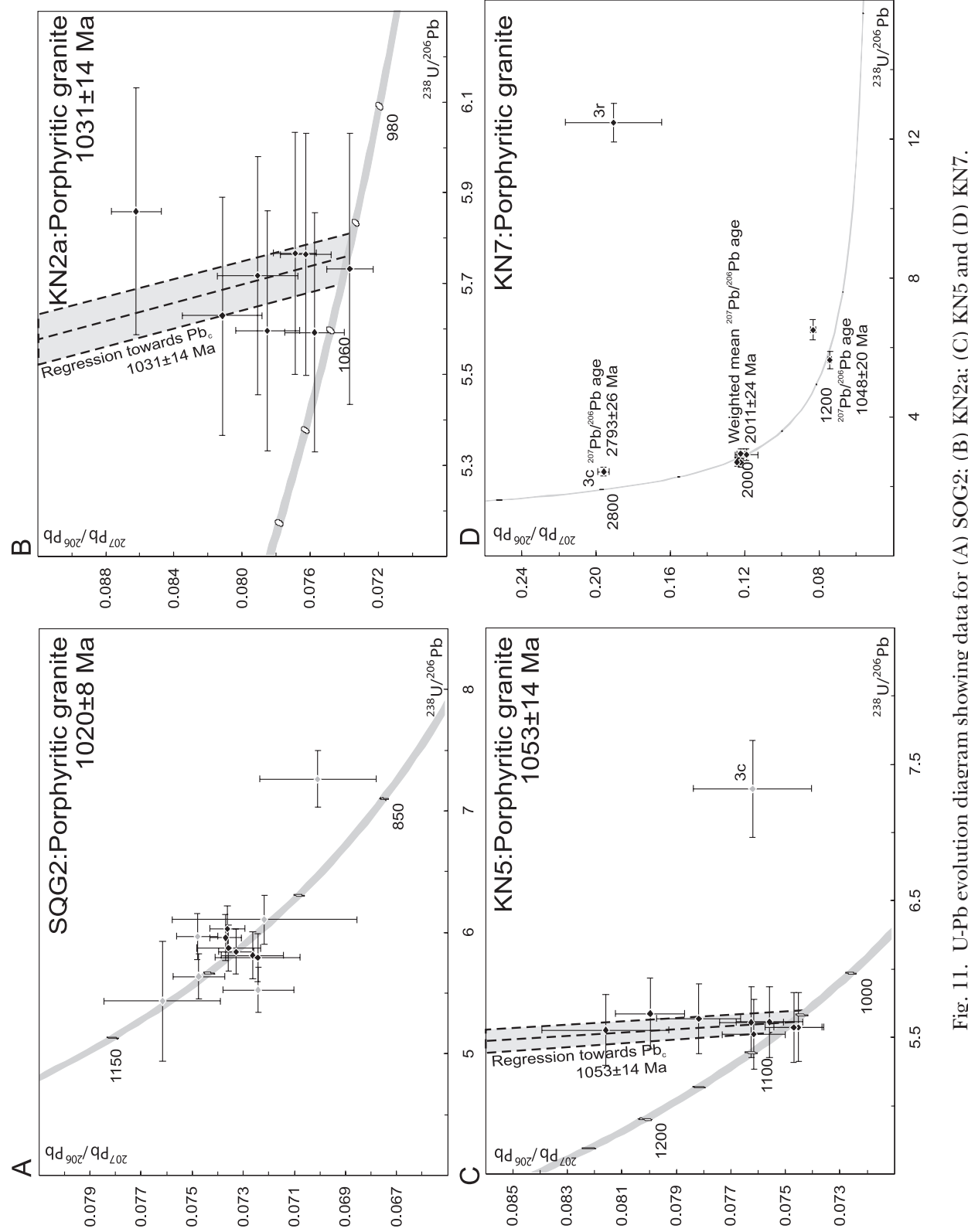
Sample KN2a-Eight analyses were conducted on eight different zircon grains, but an erratic drift normal to the Concordia curve is interpreted to reflect a problem with the common $\mathrm{Pb}$ correction (based on measured ${ }^{204} \mathrm{~Pb}$ ). The ${ }^{206} \mathrm{~Pb} /{ }^{238} \mathrm{U}$ ratios are however closely clustered and give a weighted mean age of $1041 \pm 15 \mathrm{Ma}$ (MSWD $=$ 0.51 ). The uncorrected data (as shown in fig. 11B) show a trend towards common $\mathrm{Pb}$, and a regression towards a common $\mathrm{Pb}$ composition appropriate for the age of the sample (Stacey and Kramers, 1975) yields an intercept at $1031 \pm 14 \mathrm{Ma}$ (fig. 11B; MSWD $=0.81$ ), which represents the best estimate for the crystallization age of the granite. Eight Lu-Hf isotopic analyses were conducted, recording a range of $\varepsilon_{\mathrm{Hf}}(\mathrm{t})$ values between -14.7 and -13.3 and $\mathrm{T}_{\mathrm{DM}}$ model ages between 2.09 and $2.04 \mathrm{Ga}$, indicating a significant crustal component in the granite (table 3, fig. 4).

Sample KN5 - Nine analyses were conducted on eight zircon grains, including one core-rim pair (3c and 3r). The analyzed rim contains a high amount of U (2299 $\mathrm{ppm}$ ) and is radiogenically damaged as evidenced by the significant Pb-loss and discordance (fig. 11C). The data range from discordant to concordant, and show the same drift, normal to the Concordia, as sample KN2a, indicative of incorrect $\mathrm{Pb}_{\mathrm{c}}$ correction. The values, plotted uncorrected for common $\mathrm{Pb}$ (fig. 11C), can be regressed towards common $\mathrm{Pb}$ to yield an intercept at $1053 \pm 14 \mathrm{Ma}(\mathrm{MSWD}=0.24)$ providing the best estimate for the crystallization age of the granite.

Sample KN7-Eight analyses were conducted on seven zircon grains. One core and rim pair from the same grain yielded no significant difference in ${ }^{207} \mathrm{~Pb} /{ }^{206} \mathrm{~Pb}$ age (analyses $3 \mathrm{c}$ and $3 \mathrm{r}$, table 2 ) but the rim analysis records anomalously high $\mathrm{U}$ and $\mathrm{Th}$ contents of 2878 and 39994 ppm respectively, and is extremely discordant (fig. 11D). The core, though discordant, defines a ${ }^{207} \mathrm{~Pb} /{ }^{206} \mathrm{~Pb}$ age of $2793 \pm 26 \mathrm{Ma}$, taken to be the minimum age of this xenocryst. Four analyzed zircon define a broad age group, for which a weighted mean ${ }^{207} \mathrm{~Pb} /{ }^{206} \mathrm{~Pb}$ age of $2011 \pm 24 \mathrm{Ma}$ can be calculated (fig. 11D) interpreted to represent a second xenocrystic component. Analysis 7 yields a concordant ${ }^{207} \mathrm{~Pb} /{ }^{206} \mathrm{~Pb}$ age of $1048 \pm 20 \mathrm{Ma}$ and is interpreted to provide the only estimate for the crystallization age of the granite.

Sample KN8 -Nine single and two core-rim pair analyses were conducted on complex zircon. Core analysis $1 \mathrm{c}$ yielded a concordant ${ }^{207} \mathrm{~Pb} /{ }^{206} \mathrm{~Pb}$ age of $2052 \pm 26$ $\mathrm{Ma}$ and confirms the presence of a ca. $2.05 \mathrm{Ga}$ xenocrystic component (fig. 12A). Another core $(4 \mathrm{c})$ yielded discordant isotopic ratios and has a ${ }^{207} \mathrm{~Pb} /{ }^{206} \mathrm{~Pb}$ age of $2075 \pm 70 \mathrm{Ma}$ adding further weight to the interpretation of a Paleoproterozoic source at depth. Two strongly discordant data points $1 \mathrm{r}$ and 7 yielded ${ }^{207} \mathrm{~Pb} /{ }^{206} \mathrm{~Pb}$ ages of $1320 \pm 31 \mathrm{Ma}$ and $1626 \pm 61 \mathrm{Ma}$, but because of their high discordance neither of these analyses lend themselves to simple interpretation. The remaining nine data points display an erratic drift normal to the Concordia curve, interpreted to reflect incorrect correction for common $\mathrm{Pb}$. Uncorrected data, plotted on a Tera-Wasserburg Concordia diagram (fig. 12A), yield a regression towards common $\mathrm{Pb}$, with an intercept at $1022 \pm 16 \mathrm{Ma}(\mathrm{MSWD}=1.40)$ which we consider the best estimate for the crystallization of the granite.

Sample CHT6 - Nine analyses were conducted on nine zircon grains. The zircon are characterized by relatively low $\mathrm{U}$ and $\mathrm{Th}$. Despite low counts on ${ }^{204} \mathrm{~Pb}$, values of $f_{206}$ are therefore quite high, exceeding 1 percent in 4 analyses. The data give a Concordia age of $988 \pm 23 \mathrm{Ma}(\mathrm{MSWD}=1.5$, fig. 12B), taken as the best estimate of the crystallization age of sample CHT6.

Sample ZM36-Ten zircon grains were analyzed, giving variable $\mathrm{U}$, Th and Th/U values (table 2). A Concordia age of $1028 \pm 12$ Ma can be calculated on all data (MSWD $=0.01$, fig. 12C) considered the best age estimate for the Mununga Granite. Twelve Lu-Hf isotopic analyses were conducted, recording a range of $\varepsilon_{\mathrm{Hf}}(\mathrm{t})$ values between -16.4 and -11.5 , as well as one very negative value of -23.1 , and $\mathrm{T}_{\mathrm{DM}}$ model 


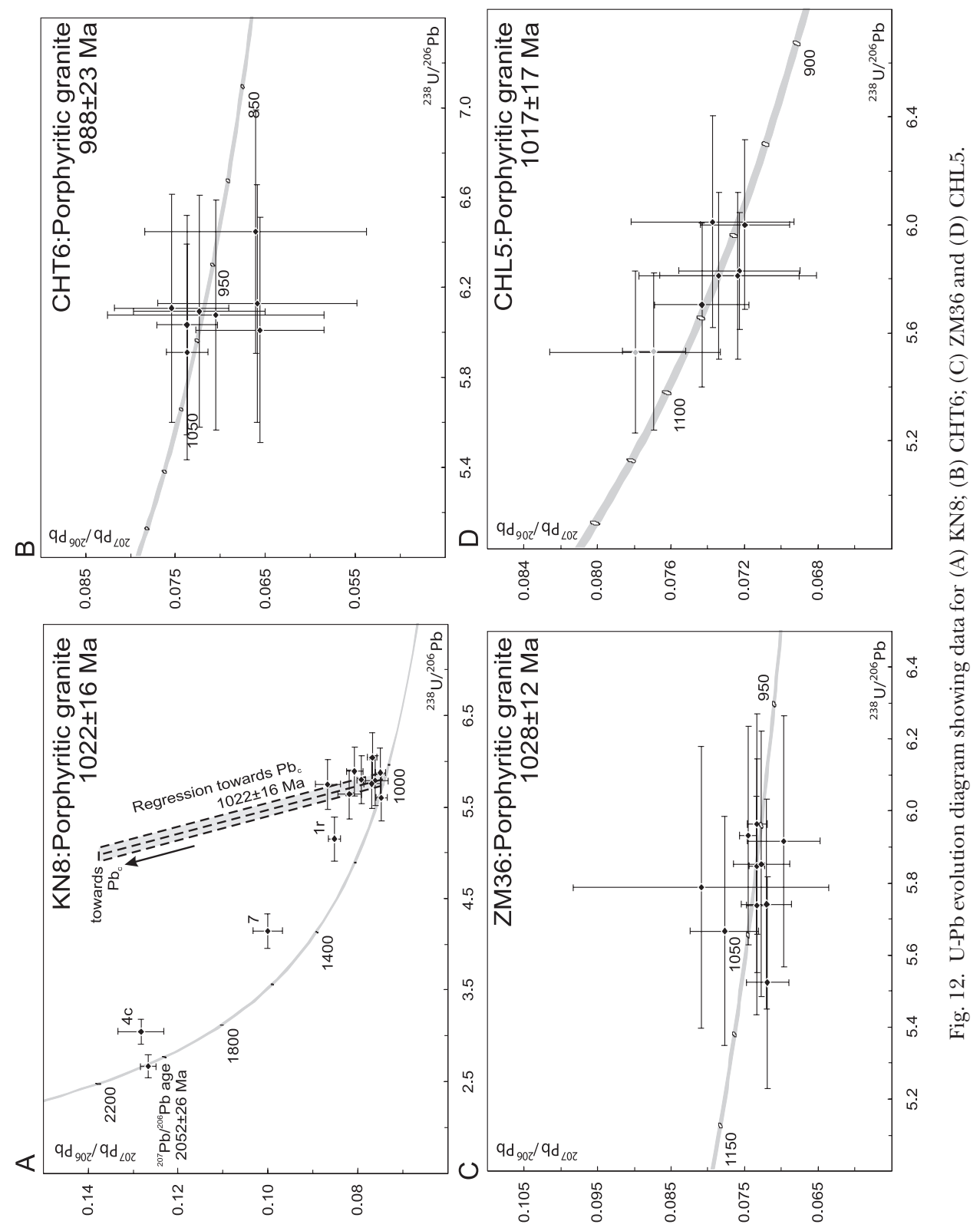


ages between 2.14 and $1.95 \mathrm{Ga}$ (2.42 Ga for the outlier), indicating crustally derived granite (table 3, fig. 4).

Sample CHL5-Eight analyses were conducted on eight different zircon and display variable $\mathrm{U}+\mathrm{Th}$ content, ranging from 288 to $914 \mathrm{ppm}$. All data give a Concordia age of $1038 \pm 15 \mathrm{Ma}(\mathrm{MSWD}=2.8$, fig. 12D). Two data point, however, plot well away from the cluster and correspond to zircon grains with anomalously high $\mathrm{Th} / \mathrm{U}$ ratios (table 2). A Concordia age on the remaining six data points gives $1017 \pm$ $17 \mathrm{Ma}(\mathrm{MSWD}=0.05)$, which we interpret as the best estimate for the emplacement age of the granite.

Sample LW1-Only six analyses were conducted on six grains and yielded a wide range of $U$ and $T h$ contents as well as apparent ages (table 2). The youngest zircon yielded a concordant ${ }^{207} \mathrm{~Pb} /{ }^{206} \mathrm{~Pb}$ age of $1005 \pm 43 \mathrm{Ma}$ and represents the only estimate for the emplacement of the granite (fig. 13A). A group of three nearconcordant analyses yield a Concordia age of $1526 \pm 12(\mathrm{MSWD}=0.07)$, and could represent xenocrysts from a granitic protolith of that age at depth.

Sample LW2-Eight analyses were conducted on eight different zircon (table 2), and yield a weighted mean ${ }^{207} \mathrm{~Pb} /{ }^{206} \mathrm{~Pb}$ age of $953 \pm 19 \mathrm{Ma}(\mathrm{MSWD}=0.62)$. A Concordia age can be calculated for three near concordant points $(4,5$ and 6$)$ which yields a more tightly constrained age of $942 \pm 9 \mathrm{Ma}(\mathrm{MSWD}=0.39$, fig. 13B $)$, which we take as the best estimate for the age of the syeno-granite in sample LW2.

Sample MTG4-Five core/rim pairs, one core and two rim analyses were completed on this sample. In two cases (9 and 10), both core and rim yielded the same result (within error), while three core/rim pairs (1c, 1r, 4c, 4r, 8c and 8r) yielded significant differences in age (table 2). Of the four older cores analyzed, three cores (1c, 4c and 7c) yielded ${ }^{207} \mathrm{~Pb} /{ }^{206} \mathrm{~Pb}$ ages of $2046 \pm 21 \mathrm{Ma}, 2017 \pm 34 \mathrm{Ma}$ and $2010 \pm 15$ Ma (fig. 13C). The first one of those corresponds to an inversely discordant point, while the two latter are concordant and correspond to a Concordia age of $2004 \pm 14$ Ma (MSWD $=6.2)$. All these cores are interpreted to represent xenocrystic components. The remaining core $(8 \mathrm{c})$ yielded a ${ }^{207} \mathrm{~Pb} /{ }^{206} \mathrm{~Pb}$ age of $1425 \pm 94 \mathrm{Ma}$, and represents an additional xenocrystic component. The remaining data plot in a tight cluster on the U-Pb evolution diagram (fig. 13C) and define a single age population with weighted mean ${ }^{207} \mathrm{~Pb} /{ }^{206} \mathrm{~Pb}$ age of $1019 \pm 13 \mathrm{Ma}$. Excluding data point $5 \mathrm{c}$, on the basis of its high $f_{206}$ value of 4.33 percent (table 2), a Concordia age of $1006 \pm 8$ Ma can be calculated $($ MSWD $=0.33)$. These ages are calculated from both core and rim analyses indicating that growth of low ${ }^{232} \mathrm{Th} /{ }^{238} \mathrm{U}$ rims happened very soon after crystallization of magmatic zircon. In conclusion, the Chilubanama Granite of sample MTG4 appears to have sampled sources of an estimated age $\sim 2050$ to 2000 and 1425 $\mathrm{Ma}$, and was emplaced at around $1006 \pm 8 \mathrm{Ma}$ coeval with regional metamorphism.

Sample ZM32-A total of six analyses were conducted on six zircon (table 2 ) and record a wide variety of apparent ages. ${ }^{207} \mathrm{~Pb} /{ }^{206} \mathrm{~Pb}$ ages of $2036 \pm 61,1859 \pm 39 \mathrm{Ma}$ and $1649 \pm 45 \mathrm{Ma}$ are recorded on zoned cores and interpreted to represent various xenocrystic components (fig. 13D). The remaining two zircons record poorly constrained ${ }^{207} \mathrm{~Pb} /{ }^{206} \mathrm{~Pb}$ ages of $946 \pm 50 \mathrm{Ma}$ and $853 \pm 85 \mathrm{Ma}$ and have relatively high $f_{206}(1.02$ and $0.75 \%$ respectively) and are therefore difficult to interpret.

DISCUSSION

\section{Pre-Irumide Magmatism}

The data presented here has shown that the Irumide Belt includes a poly-modal granitic basement comprising an Archean component dated at $2726 \pm 36 \mathrm{Ma}$ and Paleoproterozoic units dated between 2050 and $1930 \mathrm{Ma}$. This basement is unconformable, and in places structurally overlain by the ca. 1.8 Ga Muva Supergroup (De Waele and Fitzsimons, 2007). Within the Irumide Belt, the basement and supracrustal units 

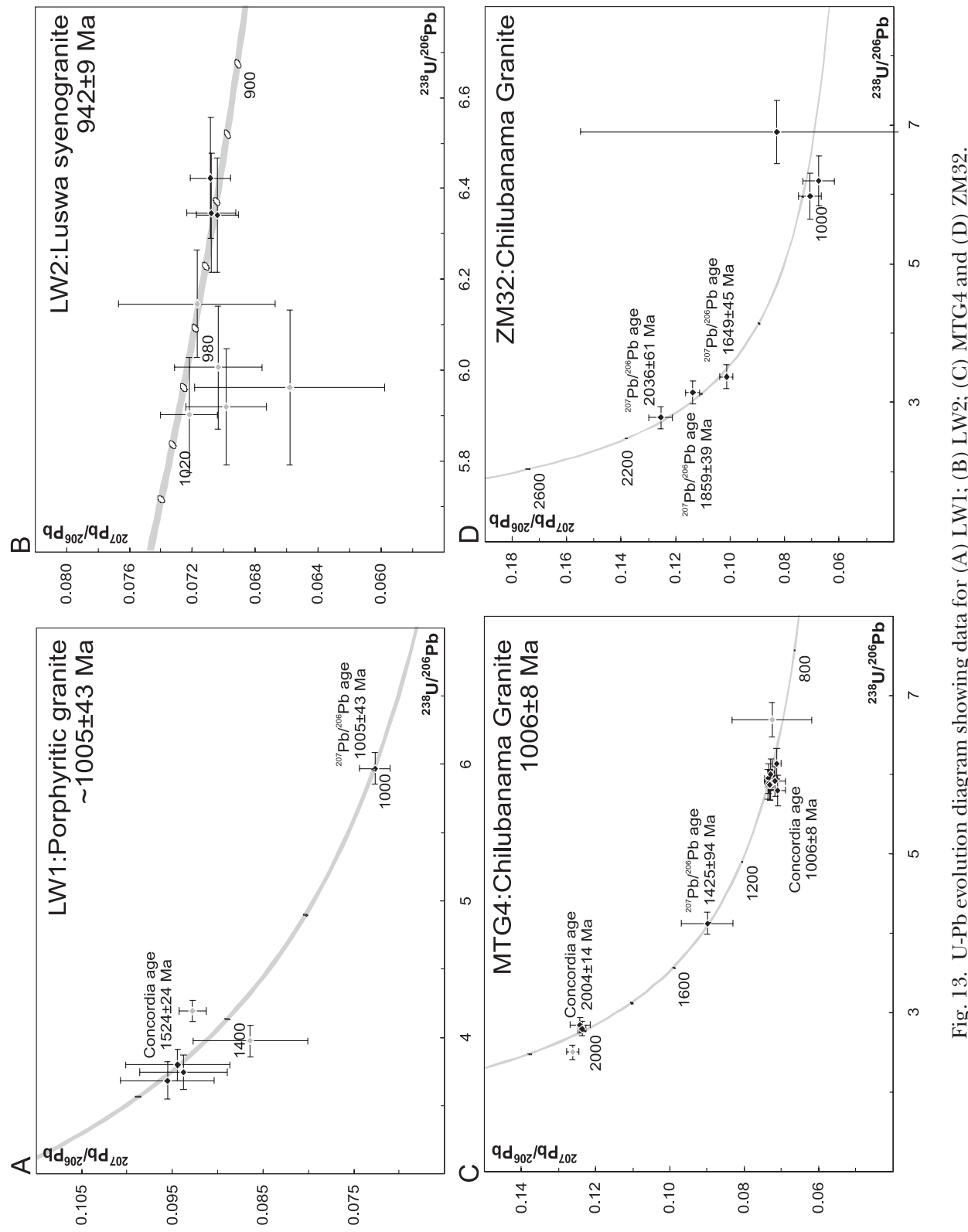
were intruded by Labradorian-aged granitoids dated between 1665 and $1630 \mathrm{Ma}$ in the southwest and between 1590 and $1530 \mathrm{Ma}$ in the northeast. These 1.65 to $1.53 \mathrm{Ga}$ granitoid rocks provide among the first evidence of Labradorian-aged magmatic activity in Africa, and provide an interesting marker against which to investigate possible similarities between the tectono-thermal history of the Congo Craton and other regions of the world. Broadly similar-aged plutons are recorded in the Epupa Complex across the Angola-Nambian border (Seth and others, 1998, 2003, 2005; Kröner and others, 2004), but none of the ages reported overlap with the 1.65 to 1.53 Ga age window recognized in the Irumide Belt.

Labradorian-aged magmatism is widely recognized in the type area of the Grenville Belt of Laurentia (Rivers, 1997; Rivers and Corrigan, 2000; Gower and Krogh, 2002) and the southward extension of the province into Mexico (Mazatzal Province, see Karlstrom and others, 2001; Rämo and others, 2003). However, both provinces are characterized by a number of magmatic episodes directly following the $1.6 \mathrm{Ga}$ magmatic event, in contrast to the distinct gap of magmatic activity in the Irumide Belt.

Another region with well-documented $1.6 \mathrm{Ga}$ magmatism is the Baltic Shield. There, a series of accretionary events make up the Gothian Orogen, spanning the period between 1.65 and $1.42 \mathrm{Ga}$, and followed by a series of extensional events between 1.34 and 1.21 Ga (Gorbatschev and Bogdanova, 1993; Andersson and others, 1999; Åhäll and others, 2000; Andersson, ms, 2000). Palaeomagnetic data for Baltica and Laurentia at 1265 Ma reported in Pesonen and others (2003), argue for the alignment of the Gothian-Mazatzal-Labradorian Belts along a large accretionary margin, a configuration which would explain the broadly coeval thermo-tectonic evolution of these belts prior to $1.5 \mathrm{Ga}$, as well as the active continental margin setting for both the Grenville and Sveconorwegian margins at 1.26 Ga (Bingen and others, 2002). As was the case for Laurentia, the margin of the Baltic Shield is characterized by a much more protracted and punctuated Mesoproterozoic geological evolution than that of the margin of the Congo Craton, making a potential link between the two between 1.6 and 1.2 Ga unlikely. The southwestern parts of the Amazonian Craton also record various episodes of granitic magmatism, which have prompted many authors to consider a connection between Amazonia, Baltica and Laurentia in a contiguous landmass (see Pesonen and others, 2003). Again, the magmatic record along the margin of Amazonia is far more extensive than that recognized along the Congo margin, making a direct link between the two in the period 1.6 to $1.1 \mathrm{Ga}$ unlikely.

In summary, a comparison of the southern margin of the Congo Craton with cratonic margins that record magmatism at about 1.6 Ga elsewhere indicates a marked absence of magmatic, thermal or tectonic events within the Irumide Belt between 1.55 $\mathrm{Ga}$ and the onset of Irumide tectonism at $1.02 \mathrm{Ga}$, in stark contrast with those from other regions. Given the fact that the ages reported in this and in previous studies cover the entire strike length of the Irumide Belt, this hiatus in geological activity is likely to be a true characteristic of this orogen. With the absence of clear rift deposits post-dating the Labradorian-aged $(1.6 \mathrm{Ga})$ events in the Irumide Belt, this makes any link between the Congo Craton and other regions with known 1.6 Ga magmatism prior to the assembly of Rodinia unlikely.

\section{Timing and Extent of Irumide Magmatism}

On the basis of this study, it can be stated that the Irumide Orogeny was characterized by voluminous granitic (s.l.) magmatism, which makes up about 70 percent of the magmatic rocks recognized in the belt. This magmatism took place between $1053 \pm 14$ and $942 \pm 9 \mathrm{Ma}$, and defined a major magmatic pulse with a calculated weighted mean age of $1015 \pm 18 \mathrm{Ma}$, corresponding closely to the reported timing of peak metamorphism at $1020 \mathrm{Ma}$ (De Waele, ms, 2005). It is worth noting that the youngest ages seem to be recorded in the northeastern Irumide Belt. A weighted 
mean age of $993 \pm 30 \mathrm{Ma}$ can be obtained for sample CHT6 and those northeast of that, while a weighted mean age on all samples southwest of CHT6 yields $1032 \pm 4 \mathrm{Ma}$. This may record some diachronicity along the strike-length of the Irumide Belt. Of the twenty six different granitoid bodies dated in the Irumide Belt, eighteen (70\%) fall within fifteen million years of peak metamorphism at $1.02 \mathrm{Ga}$. The ca. $1.02 \mathrm{Ga}$ granitoid bodies range from undeformed to strongly deformed, indicating heterogeneous distribution of strain during contractional tectonics. The widespread coverage of the sample set presented here, together with previously published and unpublished data (De Waele, ms, 2005; Johnson and others, 2005a, 2005b; De Waele and others, 2006a, 2006b, 2008; De Waele and Fitzsimons, 2007) suggest it is unlikely that additional magmatic pulses will be identified within the Irumide Orogen. As a result, the age histogram in figure $14 \mathrm{~A}$ is considered to represent a reliable fingerprint for the Irumide Orogen, against which other orogenic belts can be compared.

\section{Implications of Xenocrystic Zircon and Lu-Hf data}

Thirty of the analyses carried out in the course of this study are interpreted to represent xenocrystic zircon. These include analyses on core of complex grains, as well as single magmatic grains that yield ages older than the inferred crystallization age. The analytical data for xenocrystic zircon are tabulated in table 4 and a histogram of those with concordant $( \pm 5 \%){ }^{207} \mathrm{~Pb} /{ }^{206} \mathrm{~Pb}$ ages is shown in figure $14 \mathrm{~B}$. Since the Irumide granitoids intrude both basement and supracrustal units, either of those could represent sources for the xenocrysts. However, detrital analyses conducted on the supracrustal successions (Rainaud and others, 2003; De Waele, ms, 2005; De Waele and Fitzsimons, 2007) show that the successions in the Irumide Belt have no zircon younger than $1.8 \mathrm{Ga}$.

The zircon xenocrysts document a main population between 2.05 and $1.95 \mathrm{Ga}$, corresponding to both the main detrital zircon population reported for the Muva Supergroup and the reported age of known basement units in the Irumide Belt (De Waele, ms, 2005; Rainaud and others, 2005; De Waele and Fitzsimons, 2007). Additional small populations of xenocrystic zircon with ages of $2.7 \mathrm{Ga}, 1.86$ to $1.84 \mathrm{Ga}$ and 1.65 to $1.64 \mathrm{Ga}$ correspond respectively to the oldest known part of the basement in the Irumide Belt (Kapiri Mposhi Granite Gneiss, 2.73 Ga, De Waele, ms, 2005), the volcanic units within the Muva Supergroup (1.88-1.85 Ga, De Waele, ms, 2005; De Waele and others, 2006b) and late-Paleo- to early Mesoproterozoic granitoid rocks in the Irumide Belt (Lukamfwa Granite, 1.65-1.64 Ga, De Waele, ms, 2005; De Waele and others, 2003, 2006b). In the northeastern Irumide Belt, several xenocrystic zircon record ages between 1.54 and $1.35 \mathrm{Ga}$, for which no magmatic source is known. These may either represent evidence for an as yet unidentified cryptic magmatic terrane below the northeastern Irumide Belt, or, more likely, represent preserved detrital zircon from the Kasama Formation further north where a single concordant zircon was dated at $1.43 \mathrm{Ga}$ (De Waele and Fitzsimons, 2007). In summary, the xenocrystic zircon record confirms the presence of pre-Irumide basement units throughout the entire strike-length of the belt, supporting the notion that the Irumide Belt represents a thrust system developed along the southern margin of an Archean to Paleoproterozoic crustal block. The Lu-Hf data presented in this paper support the presence of Archean crust within and below the Irumide Belt. Median $\varepsilon_{\mathrm{Hf}}(\mathrm{t})$ values are generally strongly negative for the Irumide granitoid rocks $(\sim 1.0 \mathrm{Ga})$, in the range -14.6 to -11.3 (table 3 ), indicating that they represent crustal melts with little or no juvenile input. The median Hf model ages for these units are within a very narrow range, 1.96 to $2.08 \mathrm{Ga}$ (table 3), consistent with their derivation through total remelting of the Mkushi Gneiss and its age equivalents in the Irumide Belt. The $\sim 1.6 \mathrm{Ga}$ granitoid rocks have much more variable $\varepsilon_{\mathrm{Hf}}(\mathrm{t})$ values and range from juvenile, with positive $\varepsilon_{\mathrm{Hf}}(\mathrm{t})$, to slightly reworked with slightly negative $\varepsilon_{\mathrm{Hf}}(\mathrm{t})$ (table 3 ). Hf model ages for the $\sim 1.6 \mathrm{Ga}$ 


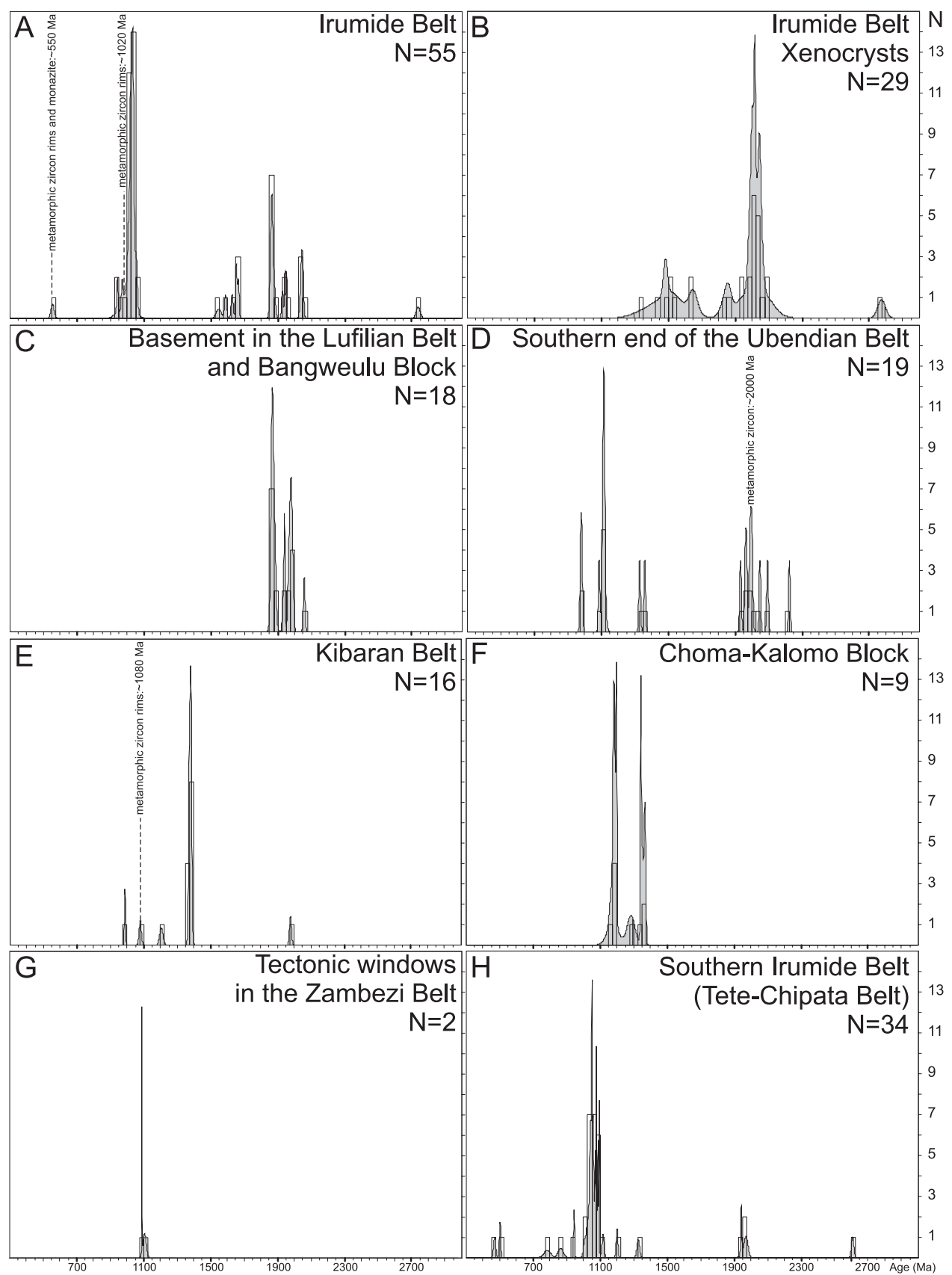

Fig. 14. Combined histograms and probability density diagrams of (A) emplacement ages of granitoid rocks in the Irumide Belt; (B) zircon ${ }^{207} \mathrm{~Pb} /{ }^{206} \mathrm{~Pb}$ crystallization ages of xenocrysts in granitoid rocks in the Irumide Belt (age data is tabulated in table 4); (C) emplacement ages of basement in the Lufilian Belt and Bangweulu Block; (D) emplacement ages of granitoid rocks in the southern end of the Ubendian Belt; (E) emplacement ages of granitoid rocks in the Kibaran Belt; (F) emplacement ages of granitoid rocks in the Choma-Kalomo Block; $(\mathrm{G})$ emplacement ages of granitoid rocks exposed in basement windows in the Zambezi Belt; $(\mathrm{H})$ emplacement and metamorphic ages of granitoid rocks in the Southern Irumide Belt. Refer to tables 2 and 5 for age data and sources. The bin size for all histograms is 25 Million years. 
TABLE 4

Zircon U-Pb ages of xenocrysts from magmatic rocks of the Irumide Belt

\begin{tabular}{lcccc}
\hline Sample & Name & $\begin{array}{c}{ }^{207} \mathbf{P b}^{\mathbf{2 0 6}} \mathbf{P b} \text { age } \\
(\mathbf{M a})\end{array}$ & $\begin{array}{c}\text { Concordance } \\
(\mathbf{\%})\end{array}$ & $\begin{array}{c}\text { Age } \\
\mathbf{( M a )}\end{array}$ \\
\hline LW1 & Porphyritic granite & $1348 \pm 66$ & 107.6 & $1005 \pm 21$ \\
MTG4 & Chilubanama Granite & $1425 \pm 47$ & 98.4 & $1010 \pm 22$ \\
LW1 & Porphyritic granite & $1483 \pm 12$ & 93.2 & $1005 \pm 21$ \\
LW1 & Porphyritic granite & $1503 \pm 46$ & 101.7 & $1005 \pm 21$ \\
LW1 & Chilubanama Granite & $1516 \pm 56$ & 99.6 & $1005 \pm 21$ \\
LW1 & Porphyritic granite & $1539 \pm 48$ & 100.8 & $1005 \pm 21$ \\
FW2 & Aplite in porphyritic granite & $1637 \pm 46$ & 102.2 & $1038 \pm 17$ \\
ZM32 & Chilubanama Granite & $1649 \pm 23$ & 101.5 & $\sim 1000$ \\
FW2 & Aplite in porphyritic granite & $1837 \pm 28$ & 90.7 & $1038 \pm 17$ \\
ZM32 & Chilubanama Granite & $1859 \pm 20$ & 95.8 & $\sim 1000$ \\
KN7 & Porphyritic granite & $1944 \pm 46$ & 97.9 & $1048 \pm 10$ \\
FW2 & Aplite in porphyritic granite & $1945 \pm 39$ & 105.4 & $1038 \pm 17$ \\
KN7 & Porphyritic granite & $1987 \pm 16$ & 94.9 & $1048 \pm 10$ \\
KN7 & Porphyritic granite & $1988 \pm 9$ & 102.4 & $1048 \pm 10$ \\
SER6-4 & Porphyritic granite & $2000 \pm 6$ & 102.7 & $1036 \pm 13$ \\
FW2 & Aplite in porphyritic granite & $2010 \pm 21$ & 101.3 & $1038 \pm 17$ \\
MTG4 & Chilubanama Granite & $2010 \pm 8$ & 98.3 & $1010 \pm 22$ \\
FW2 & Aplite in porphyritic granite & $2017 \pm 10$ & 102.6 & $1038 \pm 17$ \\
MTG4 & Chilubanama Granite & $2017 \pm 17$ & 96.6 & $1010 \pm 22$ \\
KN7 & Porphyritic granite & $2017 \pm 4$ & 100.5 & $1048 \pm 10$ \\
FW2 & Aplite in porphyritic granite & $2032 \pm 28$ & 99.4 & $1038 \pm 17$ \\
FW2 & Aplite in porphyritic granite & $2034 \pm 31$ & 97.7 & $1038 \pm 17$ \\
ZM32 & Chilubanama Granite & $2036 \pm 23$ & 97.2 & $\sim 1000$ \\
FW2 & Aplite in porphyritic granite & $2044 \pm 8$ & 99.5 & $1038 \pm 17$ \\
MTG4 & Chilubanama Granite & $2046 \pm 11$ & 106.1 & $1010 \pm 22$ \\
KN8 & Porphyritic granite & $2052 \pm 13$ & 99.7 & $1022 \pm 16$ \\
FW2 & Aplite in porphyritic granite & $2096 \pm 38$ & 102.1 & $1038 \pm 17$ \\
FW2 & Aplite in porphyritic granite & $2098 \pm 65$ & 95.5 & $1038 \pm 17$ \\
FW2 & Aplite in porphyritic granite & $2774 \pm 23$ & 99.8 & $1038 \pm 17$ \\
\hline & & &
\end{tabular}

granitoid units are in the range 1.94 to $2.22 \mathrm{Ga}$, indicating crustal residence times of at least 400 Million years. The oldest granitoids $(2.0 \mathrm{Ga})$ record $\varepsilon_{\mathrm{Hf}}(\mathrm{t})$ values of -0.1 and +2.0 , and Hf-model ages of 2.24 and $2.40 \mathrm{Ga}$, indicating a dominant juvenile character. Although the data confirm earlier suggestions of older crust below the Irumide Belt (De Waele and others, 2006b), the zircon Lu-Hf data seem to suggest that this crust on average has a mid-Paleoproterozoic age. Hf-model ages overall are in the range 1.94 to $2.40 \mathrm{Ga}$, with a median model age at $2040 \mathrm{Ma}$. We interpret this to reflect the pervasive presence of a 2.0 Ga-old juvenile crust in the Irumide Belt, with minor older Archean components, that was remelted several times to yield the $1.6 \mathrm{Ga}$ and 1.0 Ga suites. Juvenile additions to the crust within the Irumide Belt are low, in keeping with the interpretation it represents the reworked margin of an Archean-Paleoproterozoic craton (the Bangweulu Craton, which forms part of the greater Congo Craton).

\section{Regional Correlations}

The Extent of the Irumide Belt.-A possible continuation of the Irumide Belt to the northwest is masked by Neoproterozoic Katangan strata and Recent sedimentary cover. Available age data for the Bangweulu Block foreland northwest of these successions, and age data for basement units within the Lufilian Belt, indicate magmatic activity 
between 2.05 and $1.85 \mathrm{Ga}$ (data summarized in table 5, and histogram shown in fig. 14C), with a notable absence of any Mesoproterozoic magmatism. This suggests that the magmatic front of the Irumide Orogen does not extend much to the northwest of the present outcrop of the belt. The northeastern limit of the Irumide Belt is defined by a large megashear [Mugesse Shear Zone (MSZ), see fig. 1B] within the southern part of the Paleoproterozoic Ubendian Belt (Daly and others, 1985a, 1985b; Daly, ms, 1986). To the northeast of that, no age data are available to unequivocally demonstrate the termination of the Irumide Belt along this megashear. Age data for the southern part of the Ubendian Belt (Dodson and others, 1975; Ring and others, 1997, 1999; Vrána and others, 2004) demonstrate the widespread occurrence of Paleoproterozoic basement units aged between 2.22 and $1.93 \mathrm{Ga}$ (fig. 14D) and metamorphism at ca. 2.0 Ga (Ring and others, 1997). These Paleoproterozoic units were locally intruded by syenite and tonalite (1.36 and 1.33 Ga respectively, Vrána and others, 2004), and by several shear-bounded A-type granitoids between 1.12 and 1.09 Ga (Ring and others, 1999). A comparison of emplacement ages for the Southern Ubendian Belt (fig. 14D) and the Irumide Belt (fig. 14A), shows some similarities in the age of basement units, but no overlap in later magmatic activity, strongly suggesting that the Irumide Orogen does in fact terminate at the Mugesse Shear Zone.

A noteworthy feature of the regional geology is the apparent continuation of the northeast oriented Irumide Belt to the southwest, across the Neoproterozoic Zambezi Belt, and into the northeast-trending Choma-Kalomo Block. Apart from the similar structural trends of these terranes, previous age data, based on Rb-Sr whole-rock and bulk zircon U-Pb TIMS work (Hanson and others, 1988b), had supported the notion of a single Mesoproterozoic orogen, implying limited relative displacement during the Neoproterozoic and the formation of the Zambezi Orogen. Modern single zircon U-Pb SHRIMP dating on the Irumide Belt (De Waele and others, 2003, 2006a, 2006b; De Waele, ms, 2005) and Choma-Kalomo Block (Bulambo and others, 2004), however, disprove the previously proposed correlations and strongly suggest that the two orogenic segments were not contiguous between 1.4 and $1.0 \mathrm{Ga}$, and only became juxtaposed during the Neoproterozoic Zambezi orogeny. The evidence for this statement is shown in figures $14 \mathrm{~A}$ and $\mathrm{F}$, which show histograms of zircon $\mathrm{U}-\mathrm{Pb}$ ages reported for the Irumide Belt and Choma-Kalomo Block respectively (refer to table 5 for data). The data clearly show the mismatch of Mesoproterozoic magmatic events in the Choma-Kalomo Block and the Irumide Belt. Magmatism at ca. 1.37 to 1.34, 1.28 and 1.19 to $1.10 \mathrm{Ga}$ in the Choma-Kalomo Block (Hanson and others, 1988b; Bulambo and others, 2004) has not been recognized in the Irumide Belt, and conversely, peak magmatism at $1020 \pm 20 \mathrm{Ma}$ in the Irumide Belt has no expression in the ChomaKalomo Block. Isolated basement domes do occur between the two Mesoproterozoic provinces in tectonic windows of the Zambezi Belt (fig. 14G), but the crystallization ages of magmatic rocks in them of ca. $1.10 \mathrm{Ga}$ (Hanson and others, 1988a; Katongo and others, 2004) favor a possible link with the Choma-Kalomo Block (fig. 14F) or Southern Irumide Belt (fig. 14H, see below) rather than a genetic link with the Irumide Orogen (fig. 14A). This implies that the southern limit of the Irumide Orogen lies to the north of the Mpande Gneiss, perhaps coinciding with the Neoproterozoic Mwembeshi Shear Zone (fig. 1B).

The Irumide Belt is truncated to the southeast by sedimentary grabens of Karoo age (see fig. 1B). These younger structures, which are parallel to the structural trends of the Irumide Belt, make any correlation between the Irumide Belt and pre-Karoo rocks exposed to the southeast difficult. Regional studies (Johns and others, 1989; Oliver and others, 1998; Mapani and others, 2001, 2004; Johnson and Oliver, 2004; Johnson and others, 2005b, 2006, 2007; Macey and others, 2007; Westerhof and others, 2008) have shown that the area to the south of the Karoo graben comprises a variety of 
TABLE 5

Zircon U-Pb ages of magmatic rocks in and around the Irumide Belt and previously reported ages from the Irumide Belt

\begin{tabular}{|c|c|c|c|}
\hline Sample & Age (Ma) & Method used & Data source \\
\hline \multicolumn{4}{|l|}{ Irumide Belt } \\
\hline Kaunga Granite & $970 \pm 5$ & U-Pb TIMS & Daly, 1986 \\
\hline SER6-6; Lukusashi Migmatite & $1018 \pm 5$ & U-Pb SHRIMP & De Waele, 2005 \\
\hline SER6-7; Fukwe Migmatite & $1021 \pm 16$ & U-Pb SHRIMP & De Waele, 2005 \\
\hline MTGG-2; Mutangoshi Gneissic Granite & $1055 \pm 13$ & U-Pb SHRIMP & De Waele, 2005 \\
\hline IS20; Kachinga Tuff & $1856 \pm 4$ & U-Pb SHRIMP & De Waele and Fitzsimons, 2007 \\
\hline KB5; Katibunga Basalt & $1871 \pm 24$ & U-Pb SHRIMP & De Waele and Fitzsimons, 2007 \\
\hline ZM31; Luswa River Tuff & $1879 \pm 13$ & U-Pb SHRIMP & De Waele and Fitzsimons, 2007 \\
\hline Mkushi Gneiss & $2049 \pm 6$ & U-Pb SHRIMP & Rainaud and others, 2002 \\
\hline \multicolumn{4}{|l|}{ Southern Irumide Belt } \\
\hline Luangwa Gneiss & $1043 \pm 19$ & LA-ICP-MS & Cox and others, 2002 \\
\hline Luangwa Gneiss & $2608 \pm 14$ & LA-ICP-MS & Cox and others, 2002 \\
\hline Luangwa Gneiss & $\sim 2033$ & LA-ICP-MS & Cox and others, 2002 \\
\hline Madzimoyo xenolith & $1974 \pm 19$ & U-Pb SHRIMP & Johnson and others, 2006 \\
\hline Madzimoyo Granulite & $1076 \pm 6$ & U-Pb SHRIMP & Johnson and others, 2006 \\
\hline Madzimoyo Granulite & $1047 \pm 20$ & U-Pb SHRIMP & Johnson and others, 2006 \\
\hline Porphyritic granite & $1060 \pm 7$ & U-Pb SHRIMP & Johnson and others, 2006 \\
\hline Migmatite & $1942 \pm 5$ & U-Pb SHRIMP & Johnson and others, 2006 \\
\hline Nyamadzi Gneiss & $1961 \pm 31$ & U-Pb SHRIMP & Johnson and others, 2006 \\
\hline Nyamadzi Gneiss & $1065 \pm 13$ & U-Pb SHRIMP & Johnson and others, 2006 \\
\hline Mtanzi Gneiss & $1008 \pm 17$ & U-Pb SHRIMP & Johnson and others, 2006 \\
\hline Ultramylonitised granite & $1023 \pm 12$ & U-Pb SHRIMP & Johnson and others, 2006 \\
\hline Katanga Resources Granite & $1043 \pm 14$ & U-Pb SHRIMP & Johnson and others, 2006 \\
\hline Great East Road Migmatite & $1057 \pm 5$ & U-Pb SHRIMP & Johnson and others, 2006 \\
\hline Metadacite & $944 \pm 5$ & U-Pb SHRIMP & Johnson and others, 2006 \\
\hline Metadacite & $1083 \pm 18$ & U-Pb SHRIMP & Johnson and others, 2006 \\
\hline Mafic banded gneiss & $1051 \pm 12$ & U-Pb SHRIMP & Johnson and others, 2006 \\
\hline Leucogneiss & $1038 \pm 14$ & U-Pb SHRIMP & Johnson and others, 2006 \\
\hline K-feldspar augen gneiss & $1094 \pm 2$ & U-Pb SHRIMP & Johnson and others, 2006 \\
\hline K-feldspar augen gneiss & $1088 \pm 4$ & U-Pb SHRIMP & Johnson and others, 2006 \\
\hline Leucogneiss & $1070 \pm 3$ & U-Pb SHRIMP & Johnson and others, 2006 \\
\hline moz-12 & $502 \pm 8$ & U-Pb SHRIMP & Mänttäri and others, 2008 \\
\hline moz-15 & $1117 \pm 12$ & U-Pb SHRIMP & Mänttäri and others, 2008 \\
\hline moz-9 & $1077 \pm 2$ & U-Pb TIMS & Mänttäri and others, 2008 \\
\hline moz-8 & $470 \pm 14$ & U-Pb SHRIMP & Mänttäri and others, 2008 \\
\hline moz-10 & $1050 \pm 8$ & U-Pb TIMS & Mänttäri and others, 2008 \\
\hline moz-34 & $1327 \pm 16$ & U-Pb SHRIMP & Mänttäri and others, 2008 \\
\hline moz-18 & $1086 \pm 7$ & U-Pb TIMS & Mänttäri and others, 2008 \\
\hline moz-17 & $1047 \pm 29$ & Sm-Nd mineral & Mänttäri and others, 2008 \\
\hline moz-1 & $1201 \pm 10$ & U-Pb SHRIMP & Mänttäri and others, 2008 \\
\hline moz-13 & $1050 \pm 2$ & U-Pb TIMS & Mänttäri and others, 2008 \\
\hline moz-11 & $1041 \pm 4$ & U-Pb TIMS & Mänttäri and others, 2008 \\
\hline moz-16 & $784 \pm 36$ & U-Pb SHRIMP & Mänttäri and others, 2008 \\
\hline moz-14 & $864 \pm 30$ & Sm-Nd mineral & Mänttäri and others, 2008 \\
\hline moz-5 & $1046 \pm 20$ & U-Pb SHRIMP & Mänttäri and others, 2008 \\
\hline \multicolumn{4}{|l|}{ Bangweulu Block } \\
\hline MA1; Mansa Granite & $1860 \pm 13$ & U-Pb SHRIMP & De Waele and Fitzsimons, 2007 \\
\hline MA5; Mansa Volcanic & $1862 \pm 19$ & U-Pb SHRIMP & De Waele and Fitzsimons, 2007 \\
\hline MA2; Mansa Granite & $1862 \pm 8$ & U-Pb SHRIMP & De Waele and Fitzsimons, 2007 \\
\hline MA9; Musonda Falls Granite & $1866 \pm 9$ & U-Pb SHRIMP & De Waele and Fitzsimons, 2007 \\
\hline MA3; Mansa Volcanic & $1868 \pm 7$ & U-Pb SHRIMP & De Waele and Fitzsimons, 2007 \\
\hline \multicolumn{4}{|l|}{ Tectonic windows in the Zambezi Belt } \\
\hline Munali Granite & $1090 \pm 1$ & U-Pb TIMS & Katongo and others, 2004 \\
\hline Mpande Gneiss & $1106 \pm 19$ & $\mathrm{U}-\mathrm{Pb}$ TIMS & Hanson and others, $1988 \mathrm{a}$ \\
\hline
\end{tabular}


TABLE 5

(continued)

\begin{tabular}{|c|c|c|c|}
\hline Sample & Age (Ma) & Method used & Data source \\
\hline \multicolumn{4}{|l|}{ Choma-Kalomo Block } \\
\hline Granite (Choma-Kalomo Block) & $1174 \pm 27$ & U-Pb SHRIMP & Bulambo and others, 2004 \\
\hline Granite & $1177 \pm 70$ & U-Pb SHRIMP & Bulambo and others, 2004 \\
\hline Granite & $1181 \pm 9$ & U-Pb SHRIMP & Bulambo and others, 2004 \\
\hline Granite & $1188 \pm 11$ & U-Pb SHRIMP & Bulambo and others, 2004 \\
\hline Semahwa Gneiss & $1198 \pm 6$ & U-Pb TIMS & Hanson and others, $1988 \mathrm{~b}$ \\
\hline Chilala Gneiss & $1285 \pm 64$ & U-Pb TIMS & Hanson and others, $1988 \mathrm{~b}$ \\
\hline Zongwe Gneiss & $1343 \pm 6$ & U-Pb TIMS & Hanson and others, $1988 \mathrm{~b}$ \\
\hline Siasikabole Granite & $1352 \pm 14$ & U-Pb TIMS & Hanson and others, $1988 \mathrm{~b}$ \\
\hline Granite & $1368 \pm 10$ & U-Pb SHRIMP & Bulambo and others, 2004 \\
\hline \multicolumn{4}{|l|}{ Lufilian Belt } \\
\hline Kinsenda Lufubu Schist & $1873 \pm 8$ & U-Pb SHRIMP & Rainaud and others, 2002 \\
\hline Solwezi Granite & $1874 \pm 9$ & U-Pb TIMS & John, 2001 \\
\hline Kinsenda Granite (Luina dome) & $1882 \pm 23$ & U-Pb TIMS & Ngoyi and others, 1991 \\
\hline Kabompo Granite & $1884 \pm 10$ & U-Pb TIMS & John, 2001 \\
\hline Kabompo Dome Granite & $1934 \pm 6$ & U-Pb TIMS & Key and others, 2001 \\
\hline Kabompo Dome Granite & $1940 \pm 3$ & U-Pb TIMS & Key and others, 2001 \\
\hline Samba Porphyry & $1964 \pm 12$ & U-Pb SHRIMP & Rainaud and others, 2002 \\
\hline Lufubu Schist & $1970 \pm 10$ & U-Pb SHRIMP & Rainaud and others, 2002 \\
\hline Chambishi Granite & $1980 \pm 7$ & U-Pb SHRIMP & Rainaud and others, 2002 \\
\hline Chambishi Granite & $1983 \pm 5$ & U-Pb SHRIMP & Rainaud and others, 2002 \\
\hline Mufulira Granite & $1991 \pm 3$ & U-Pb SHRIMP & Rainaud and others, 2002 \\
\hline Mulungushi Gneiss & $1976 \pm 5$ & U-Pb SHRIMP & Rainaud and others, 2005 \\
\hline Mwinilunga Granite & $2058 \pm 7$ & U-Pb SHRIMP & Key and others, 2001 \\
\hline \multicolumn{4}{|l|}{ Kibaran Belt } \\
\hline Rumeza Granite & $1383 \pm 17$ & U-Pb SHRIMP & Tack and others, pers.comm. \\
\hline Mugere Granite & $1379 \pm 10$ & U-Pb SHRIMP & Tack and others, pers.comm. \\
\hline Mugere Migmatite & $1380 \pm 8$ & U-Pb SHRIMP & Tack and others, pers.comm. \\
\hline Kiganda Granite & $1371 \pm 7$ & U-Pb SHRIMP & Tack and others, pers.comm. \\
\hline Muramba Granite & $1380 \pm 6$ & U-Pb SHRIMP & Tack and others, pers.comm. \\
\hline Kilimbi-Muzumu Granite & $1373 \pm 6$ & U-Pb SHRIMP & Tack and others, pers.comm. \\
\hline Butare Gneiss & $1979 \pm 12$ & U-Pb SHRIMP & Tack and others, pers.comm. \\
\hline Musongati Massif Norite & $1374 \pm 14$ & U-Pb SHRIMP & Tack and others, pers.comm. \\
\hline Bukirasazi Granite & $1205 \pm 19$ & U-Pb SHRIMP & Tack and others, pers.comm. \\
\hline Kasika Sn Granite & $987 \pm 6$ & U-Pb SHRIMP & Tack and others, pers.comm. \\
\hline Kisele Monzogranite Gneiss & $1386 \pm 8$ & U-Pb SHRIMP & Kokonyangi and others, 2004 \\
\hline Kabonvia Gneiss & $1386 \pm 7$ & U-Pb SHRIMP & Kokonyangi and others, 2004 \\
\hline Nyangwa Monzogranite & $1383 \pm 5$ & U-Pb SHRIMP & Kokonyangi and others, 2004 \\
\hline Kungwe-Kalumengongo Monzogranite & $1377 \pm 10$ & U-Pb SHRIMP & Kokonyangi and others, 2004 \\
\hline Fwifwi Leucomonzogranite & $1372 \pm 10$ & U-Pb SHRIMP & Kokonyangi and others, 2004 \\
\hline Kisele Monzogranite Gneiss & $1079 \pm 14$ & U-Pb SHRIMP & Kokonyangi and others, 2004 \\
\hline \multicolumn{4}{|l|}{ Southern Ubendian Belt } \\
\hline Aplite (Luromo Granite) & $977 \pm 1$ & $\mathrm{~Pb}$ evaporation & Ring and others, 1999 \\
\hline Aplite (Wililo Granite) & $983 \pm 1$ & $\mathrm{~Pb}$ evaporation & Ring and others, 1999 \\
\hline Lwakwa Granite & $1087 \pm 11$ & U-Pb TIMS & Ring and others, 1999 \\
\hline Luromo Granite & $1108 \pm 1$ & $\mathrm{~Pb}$ evaporation & Ring and others, 1999 \\
\hline Wililo Granite & $1115 \pm 1$ & $\mathrm{~Pb}$ evaporation & Ring and others, 1999 \\
\hline Wililo Granite & $1116 \pm 1$ & $\mathrm{~Pb}$ evaporation & Ring and others, 1999 \\
\hline Wililo Granite & $1118 \pm 1$ & $\mathrm{~Pb}$ evaporation & Ring and others, 1999 \\
\hline Mwenga Granite & $1119 \pm 20$ & U-Pb TIMS & Ring and others, 1999 \\
\hline Ntendele Metatonalite & $1329 \pm 1$ & $\mathrm{~Pb}$ evaporation & Vrána and others, 2004 \\
\hline Mivula Syenite & $1360 \pm 1$ & $\mathrm{~Pb}$ evaporation & Vrána and others, 2004 \\
\hline Nyika Granite & $1932 \pm 9$ & U-Pb TIMS & Dodson and others, 1975 \\
\hline Biotite Metatonalite & $1961 \pm 1$ & $\mathrm{~Pb}$ evaporation & Vrána and others, 2004 \\
\hline Nyika Granite & $1969 \pm 1$ & $\mathrm{~Pb}$ evaporation & Ring and others, 1997 \\
\hline Rumphi Granite & $1988 \pm 1$ & $\mathrm{~Pb}$ evaporation & Ring and others, 1997 \\
\hline Chelinda Granite & $1995 \pm 1$ & $\mathrm{~Pb}$ evaporation & Ring and others, 1997 \\
\hline Luromo Granite & $2002 \pm 1$ & $\mathrm{~Pb}$ evaporation & Ring and others, 1997 \\
\hline Rumphi Granite & $2048 \pm 1$ & $\mathrm{~Pb}$ evaporation & Ring and others, 1997 \\
\hline Luromo Granite & $2093 \pm 1$ & $\mathrm{~Pb}$ evaporation & Ring and others, 1997 \\
\hline Luromo Granite & $2224 \pm 1$ & $\mathrm{~Pb}$ evaporation & Ring and others, 1997 \\
\hline
\end{tabular}


lithotectonic terrains. Johnson and others (2006) introduced the term Southern Irumide Belt to denote the area to the south of the Karoo grabens, but Westerhof and others prefer to use the name Tete-Chipata Belt (Westerhof and others, 2008). In this paper we will use the earlier nomenclature. Published and unpublished age data, reported in table 5 and shown in figure $14 \mathrm{H}$, show the presence of a Paleoproterozoic basement at about $2.0 \mathrm{Ga}$, similar to that occurring within the Irumide Belt, but indicate magmatic events spanning between 1.10 and $0.95 \mathrm{Ga}$ and accompanying peak metamorphic conditions between 1.06 and $1.05 \mathrm{Ga}$. These data clearly demonstrate that Mesoproterozoic magmatism and associated metamorphic conditions in the Southern Irumide Belt predate those in the Irumide Belt, as previously stated by Johnson and others (2006). Because of the fact that these age differences occur across strike, with no apparent gradual transition between the two terranes, it is considered unlikely that the differences can be ascribed to diachronous development of a single Irumide Orogen. Moreover, geochemical and isotopic data for magmatic rocks of the Irumide Belt (De Waele, ms, 2005; De Waele and others, 2006b) and the Southern Irumide Belt (Johnson and others, 2007; Macey and others, 2007; Mäkitie and others, 2008; Westerhof and others, 2008) clearly demonstrate significant petrological differences between the two terrains, suggesting that the Irumide Belt and Southern Irumide Belt developed separately and were juxtaposed along a suture now obscured by the Karoo graben.

The Irumide and Kibaran belts.-In the past, the Irumide Belt has often been correlated with the subparallel Kibaran Belt to the northwest, based on Rb-Sr and K-Ar data that indicate that both belts are Mesoproterozoic, and on their similar northeastoriented structural trend. The geochronological framework of the Kibaran Belt has recently been refined using SHRIMP U-Pb geochronology into two main contractional deformation events, followed by the intrusion of the tin-bearing granitoids. Kokonyangi and others (2004) reported ages of $1386 \pm 8 \mathrm{Ma}, 1385 \pm 7 \mathrm{Ma}, 1383 \pm 5 \mathrm{Ma}$, $1372 \pm 10 \mathrm{Ma}$ and $1377 \pm 10 \mathrm{Ma}$ for various phases of the Mitwaba granites in Congo D.R., while Tack and others (2002) reported ages of $1383 \pm 17 \mathrm{Ma}, 1379 \pm 10 \mathrm{Ma}$, $1371 \pm 7 \mathrm{Ma}$ and $1374 \pm 14 \mathrm{Ma}$ for granitoid rocks in the northeastern part of the Kibaran Belt. These ages tightly constrain the main Kibaran magmatic pulse between $1386 \pm 8 \mathrm{Ma}$ and $1371 \pm 7 \mathrm{Ma}$ (fig. 14E). One granite in the northeastern part of the Kibaran Belt yielded an age of $1205 \pm 19 \mathrm{Ma}$ and indicates the presence of a localized second magmatic pulse at that time (Tack and others, 2002). In the central Kibaran Belt, Kokonyangi and others (2004) reported a metamorphic zircon U-Pb SHRIMP age of $1079 \pm 14 \mathrm{Ma}$, suggesting at least a local metamorphic event around that time. These new data render a correlation between the Irumide Belt and the Kibaran Belt untenable (see histograms in figs. 14A and 14E), but bring out striking similarities between Choma-Kalomo Block (fig. 14F) and the Kibaran Belt, raising the possibility of their original continuity. Unrug (1992) pointed out that a sinistral movement of 150 to $200 \mathrm{~km}$ along the Mwembeshi Shear Zone would place the Choma-Kalomo Block in direct continuation with the Kibaran Belt, but evaluation of this prediction awaits additional work.

\section{ACKNOWLEDGMENTS}

Fieldwork and research by BDW was funded by the Australian Research Councils' Tectonics Special Research Centre and by an International Postgraduate Research Scholarship to BDW at Curtin University of Technology in Perth, Australia. BDW wishes to thank the staff at the Perth Consortium SHRIMP facilities, the Geological Society of Zambia and the Geology Department at the University of Zambia for support throughout the work. The authors wish to thank the reviewers, Drs. Toby Rivers, Simon P. Johnson and Luc Tack for constructive comments that have greatly enhanced the manuscript. This publication is a contribution to IGCP projects 418 and 440 . 


\section{REFERENCES}

Ackermann, E. H., 1950, Ein neuer faltengurtel in Nordrhodesien und seine tectonische Stellung im Afrikanishen grundgebirge: Geologische Rundschau, v. 38, p. 24-39, doi:10.1007/BF01766570.

1960, Strukturen im untergrund eines intrakratonischen Doppel-Orogens (Irumiden Nordrhodesien): Geologische Rundschau, v. 50, p. 538-553, doi:10.1007/BF01786869.

Åhäll, K.-I., Connelly, J. N., and Brewer, T. S., 2000, Episodic rapakivi magmatism due to distal orogenesis?: Correlation of 1.69-1.50 Ga orogenic and inboard, "anorogenic" events in the Baltic Shield.: Geology, v. 28, p. 823-826, doi:10.1130/0091-7613(2000)28<823:ERMDTD〉2.0.CO;2.

Andersson, J., ms, 2000, Sveconorwegian orogenesis in the southwestern Baltic Shield: Zircon geochronology and tectonothermal setting of orthogneisses in SW Sweden: Lund, Sweden, Lund University, Ph. D. thesis, $155 \mathrm{p}$.

Andersson, J., Süderlund, U., Cornell, D., Johannson, L., and Moller, C., 1999, Sveconorwegian (-Grenvillian) deformation, metamorphism and leucosome formation in SW Sweden, SW Baltic Shield: constraints from a Mesoproterozoic granite intrusion: Precambrian Research, v. 98, p. 151-171, doi:10.1016/S0301-9268(99)00048-0.

Bingen, B., Mansfeld, J., Sigmond, E. M. O., and Stein, H., 2002, Baltica-Laurentia link during the Mesoproterozoic: 1.27 Ga development of continental basins in the Sveconorwegian Orogen, southern Norway: Canadian Journal of Earth Sciences, v. 39, p. 1425-1440. doi:10.1139/e02-054.

Blichert-Toft, J., Chauvel, C., and Albarede, F., 1997, The Lu-Hf geochemistry of chondrites and the evolution of the mantle-crust system: Earth and Planetary Science Letters, v. 148, p. 243-258, doi:10.1016/ S0012-821X(97)00040-X.

Brewer, M. S., Haslam, H. W., Darbyshire, P. F. P., and Davis, A. E., 1979, Rb-Sr age determinations in the Bangweulu block, Luapula Province, Zambia: London, Institute of Geological Sciences, Report 79/5, p. 1-11.

Bulambo, M., De Waele, B., Kampunzu, A. B., and Tembo, F., 2004, SHRIMP U-Pb geochronology of the Choma-Kalomo block (Zambia) and geological implications: Orleans, France, 20th Colloquium of African Geology, 2-7 June 2004, p. 96.

Bulambo, J.-P., De Waele, B., Kampunzu, A. B., and Tembo, F., 2006, SHRIMP U-Pb geochronology of the Choma -Kalomo block (Zambia) and geological implications: Maputo, 21st Colloquium on African Geology, 03-05 July 2006, 3 p.

Cox, R. A., Rivers, T., Mapani, B., Tembo, F., and De Waele, B., 2002, New U-Pb data for the Irumide belt: LAM-ICP-MS results for Luangwa Terrane: Windhoek, Namibia, Geological Survey of Nambia, 11th IAGOD Quadrennial Symposium and Geocongress, technical meeting IGCP 440, Assembly and Breakup of Rodinia, p. 10.

Cvetcovic, D., 1973, The geology of the Mita hills area; explanation of degree sheet 1429, NW quarter: Lusaka, Geological Survey Department of Zambia, p. 12.

Daly, M. C., ms, 1986, The tectonic and thermal evolution of the Irumide belt, Zambia, Institute of African Geology: Leeds, University of Leeds, Ph. D. thesis, 326 p.

1995a, The geology of Chinsali and Mutangoshi Hills area; explanation of degree sheet 1032, SE and SW quarter: Lusaka, Geological Survey Department of Zambia.

_ 1995b, The geology of Mulilansolo Mission and Isoka areas; explanation of degree sheet 1032, NE and NW quarter: Lusaka, Geological Survey Department of Zambia.

Daly, M. C., and Unrug, R., 1982, The Muva Supergroup, northern Zambia: Transactions of the Geological Society of South Africa, v. 85, p. 155-165.

Daly, M. C., Klerkx, J., and Nanyaro, J. T., 1985a, Early Proterozoic exotic terranes and strike-slip accretion in the Ubendian belt of southwest Tanzania: Terra Cognita, v. 5, p. 257.

1985b, Early Proterozoic strike-slip accretion in the Ubendian belt of southwest Tanzania, in Bowden, P., Kinnaird, J. A., and Van, H. F. D., editors, 13th Colloquium of African geology: Centre International pour la Formation et les Echanges Géologiques (CIFEG).

De Waele, B., ms, 2005, The Proterozoic geological history of the Irumide belt, Zambia, Department of Applied Geology: Perth, Curtin University of Technology, Ph. D. thesis, 468 p.

De Waele, B., and Fitzsimons, I. C. W., 2007, The nature and timing of Palaeoproterozoic sedimentation at the southeastern margin of the Congo Craton; zircon $\mathrm{U}-\mathrm{Pb}$ geochronology of plutonic, volcanic and clastic units in northern Zambia: Precambrian Research, v. 159, p. 95-116, doi:10.1016/j. precamres.2007.06.004.

De Waele, B., and Mapani, B., 2002, Geology and correlation of the central Irumide belt: Journal of African Earth Sciences, v. 35, p. 385-397, doi:10.1016/S0899-5362(02)00149-5.

De Waele, B., Wingate, M. T. D., Mapani, B., and Fitzsimons, I. C. W., 2003, Untying the Kibaran knot: A reassessment of Mesoproterozoic correlations in southern Africa based on SHRIMP U-Pb data from the Irumide belt: Geology, v. 31, p. 509-512, doi:10.1130/0091-7613(2003)031<0509:UTKKAR>2.0.CO;2.

De Waele, B., Kampunzu, A. B., Mapani, B. S. E., and Tembo, F., 2006a, The Mesoproterozoic Irumide belt of Zambia: Journal of African Earth Sciences, v. 46, p. 36-70, doi:10.1016/j.jafrearsci.2006.01.018.

De Waele, B., Liégeois, J. P., Nemchin, A. A., and Tembo, F., 2006b, Isotopic and geochemical evidence of Proterozoic episodic crustal reworking within the Irumide Belt of south-central Africa, the southern metacratonic boundary of an Archaean Bangweulu Craton: Precambrian Research, v. 148, p. 225-256, doi:10.1016/j.precamres.2006.05.006.

De Waele, B., Johnson, S. P., and Pisarevsky, S. A., 2008, Palaeoproterozoic to Neoproterozoic growth and evolution of the eastern Congo Craton: Its role in the Rodinia puzzle: Precambrian Research, v. 160, p. 127-141, doi:10.1016/j.precamres.2007.04.020.

Dodson, M. H., Cavanagh, B. J., Thatcher, E. C., and Aftalion, M., 1975, Age limits for the Ubendian metamorphic episode in northern Malawi: Geological Magazine, v. 112, p. 403-410. 
Gorbatschev, R., and Bogdanova, S., 1993, Frontiers in the Baltic Shield: Precambrian Research, v. 64, p. 3-21, doi:10.1016/0301-9268(93)90066-B.

Goscombe, B., Armstrong, R. A., and Barton, J. M., 2000, Geology of the Chewore Inliers, Zimbabwe: Constraining the Mesoproterozoic to Palaeozoic evolution of the Zambezi belt: Journal of African Earth Sciences, v. 30, p. 589-627, doi:10.1016/S0899-5362(00)00041-5

Gower, C. F., and Krogh, T. E., 2002, A U-Pb geochronological review of the Proterozoic history of the eastern Grenville Province: Canadian Journal of Earth Sciences, v. 39, p. 795-829, doi:10.1139/e01-090.

Griffin, W. L., Pearson, N. J., Belousova, E., Jackson, S. E., van Achterbergh, E., O’Reilly, S. Y., and Shee, S. R., 2000, The Hf isotope composition of cratonic mantle: LAM-MC-ICPMS analysis of zircon megacrysts in kimberlites: Geochimica et Cosmochimica Acta, v. 64, p. 133-147, doi:10.1016/S0016-7037(99)00343-9.

Griffin, W. L., Belousova, E. A., Shee, S. R., Pearson, N. J., and O'Reilly, S. Y., 2004, Archean crustal evolution in the northern Yilgarn Craton: U-Pb and Hf-isotope evidence from detrital zircons: Precambrian Research, v. 131, p. 231-282, doi:10.1016/j.precamres.2003.12.011.

Hanson, R. E., 2003, Proterozoic geochronology and tectonic evolution of southern Africa, in Yoshida, M., Windley, B. F., and Dasgupta, S., editors, Proterozoic East Gondwana: Supercontinent Assembly and Breakup: Geological Society, London, Special Publication, v. 206, p. 427-463.

Hanson, R. E., Wilson, T. J., and Wardlaw, M. S., 1988a, Deformed batholiths in the Pan African Zambezi belt, Zambia: Age and implications for regional Proterozoic tectonics: Geology, v. 16, p. 1134-1137,

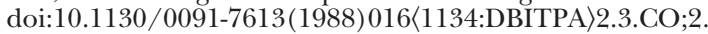

Hanson, R. E., Wilson, T. J., Brueckner, H. K., Onstott, T. C., Wardlaw, M. S., Johns, C. C., and Hardcastle, K. C., 1988b, Reconnaissance geochronology, tectonothermal evolution, and regional significance of the Middle Proterozoic Choma-Kalomo block, southern Zambia: Precambrian Research, v. 42, p. 39-61, doi:10.1016/0301-9268(88)90009-5.

Hoskin, P. W. O., and Black, L. P., 2000, Metamorphic zircon formation by solid-state recrystallization of protolith igneous zircon: Journal of Metamorphic Geology, v. 18, p. 423-439, doi:10.1046/j.15251314.2000.00266.x.

John, T., ms, 2001, Subduction and continental collision in the Lufilian Arc -Zambezi belt orogen: A petrological, geochemical, and geochronological study of eclogites and whiteschists (Zambia), Mathematisch-Naturwissenschaftlichen Fakultat: Kiel, University of Kiel, Ph. D. thesis, 78 p.

John, T., Schenk, V., and Tembo, F., 1999, The metamorphic evolution and U/Pb dating of monazites of the southern Irumide belt, SE-Zambia, in De Waele, B., Tembo, F., and Key, R. M., editors, Abstracts Volume IGCP 419/419: Lusaka, Geological Society of Zambia, p. 7.

Johns, C. C., Liyungu, K., Mabuku, S., Mwale, G., Sakungo, F., Tembo, D., Vallance, G., and Barr, M. W. C., 1989, The stratigraphic and structural framework of eastern Zambia: Results of a geotraverse: Journal of African Earth Sciences, v. 9, p. 123-136, doi:10.1016/0899-5362(89)90015-8.

Johnson, S. P., and Oliver, G. J. H., 2004, Tectonothermal history of the Kaourera Arc, northern Zimbabwe: implications for the tectonic evolution of the Irumide and Zambezi Belts of south central Africa: Precambrian Research, v. 130, p. 71-97, doi:10.1016/j.precamres.2003.10.016.

Johnson, S. P., De Waele, B., Tani, K., and Tembo, F., 2005a, Mesoproterozoic supra-subduction magmatism in the southern Irumide Belt, central southern Africa: implications for the Congo Craton in Rodinia reconstructions, Supercontinents and Earth Evolution Symposium: Fremantle, Western Australia, 26-30 September, 2005, p. 83-84.

Johnson, S. P., Rivers, T., and De Waele, B., 2005b, A Review of the Mesoproterozoic to early Palaeozoic magmatic and tectonothermal history of south-central Africa: implications for Rodinia and Gondwana: London, Journal of the Geological Society, v. 162, p. 433-450, doi:10.1144/0016-764904-028.

Johnson, S. P., De Waele, B., and Liyungu, A. K., 2006, U-Pb sensitive high-resolution ion microprobe (SHRIMP) zircon geochronology of granitoid rocks in eastern Zambia: Terrane subdivision of the Mesoproterozoic Southern Irumide Belt: Tectonics, v. 25, p. TC6004, doi:10.1029/2006TC001977.

Johnson, S. P., De Waele, B., Tembo, F., Katongo, C., Tani, K., Chang, Q., Iizuka, T., and Dunkley, D., 2007, Geochemistry, geochronology and isotopic evolution of the Chewore-Rufunsa terrane, Southern Irumide Belt. A Mesoproterozoic continental margin arc: Journal of Petrology, v. 48, p. 1411-1441, doi:10.1093/petrology/egm025.

Karlstrom, K. E., Åhäll, K.-I., Harlan, S. S., Williams, M. L., McLelland, J., and Geissman, J. W., 2001, Long-lived $(1.8-1.0 \mathrm{Ga})$ convergent orogen in southern Laurentia, its extensions to Australia and Baltica, and implications for refining Rodinia: Precambrian Research, v. 111, p. 5-30, doi:10.1016/S03019268(01)00154-1.

Katongo, C., Köller, F., Klötzli, U., Koeberl, C., Tembo, F., and De Waele, B., 2004, Petrography, geochemistry and geochronology of granitoid rocks in the Neoproterozoic-Paleozoic Lufilian-Zambezi belt, Zambia: Implications for tectonic setting and regional correlation: Journal of African Earth Sciences, v. 40, p. 219-244, doi:10.1016/j.jafrearsci.2004.12.007.

Key, R. M., Liyungu, A. K., Njamu, F. M., Somwe, V., Banda, J., Mosley, P. N., and Armstrong, R. A., 2001, The western arm of the Lufilian Arc in NW Zambia and its potential for copper mineralization: Journal of African Earth Sciences, v. 33, p. 503-528, doi:10.1016/S0899-5362(01)00098-7.

Kokonyangi, J., Armstrong, R. A., Kampunzu, A. B., Yoshida, M., and Okudaira, T., 2004, U-Pb zircon geochronology and petrology of granitoids from Mitwaba (Katanga, Congo): implications for the evolution of the Mesoproterozoic Kibaran belt: Precambrian Research, v. 132, p. 79-106, doi:10.1016/ j.precamres.2004.02.007.

Kröner, S., Konopásek, J., Kröner, A., Passchier, C. W., Poller, U., Wingate, M. T. D., and Hoffman, K. H., 2004, U-Pb and Pb-Pb zircon ages for metamorphic rocks in the Kaoko Belt of Northwestern Namibia: A Palaeo- to Mesoproterozoic basement reworked during the Pan-African orogeny: South African Journal of Geology, v. 107, p. 455-476, doi:10.2113/107.3.455. 
Ludwig, K. R., 2001a, Isoplot/Ex rev. 2.49: Berkeley, California, Berkeley Geochronology Center, p. 54. 2001b, Squid 1.02: A User's Manual: Berkeley, Berkeley Geochronology Center, p. 19.

Macey, P. H., Ingram, B. A., Cronwright, M. S., Botha, G. A., Roberts, M. R., Grantham, G. H., Maree, L. P., Botha, P. M. W., Kota, M., Opperman, R., Haddon, I. G., Nolte, J. C., and Rower, M., 2007, Map Explanation of Sheets Alto Molócuè (1537), Murrupula (1538), Nampula (1639), Mogincual (1540), Errego (1637), Gilé (1638) and Angoche (1639-40): Maputo, National Directorate of Geology, Mozambique, p. 402.

Mäkitie, H., Lehtonen, M., I., Manninen, T., Marques, J. M., Cune, G., and Mavíe, H., 2008, Petrography and Geochemistry of Granitoid Rocks in the Northern Part of Tete Province, Mozambique, in Pekkala, Y., Lehto, T., and Mäkitie, H., editors, GTK Consortium Geological Surveys in Mozambique 2002-2007: Geological Survey of Finland, Special Paper 48, p. 167-189.

Mapani, B., 1999, Tectonic and metamorphic evolution of the Serenje and adjoining areas, in De Waele, B., Tembo, F., and Key, R. M., editors, Abstracts Volume IGCP 418/419: Lusaka, Geological Society of Zambia, p. 16.

Mapani, B. S. E., Rivers, T., Tembo, F., and Katongo, C., 2001, Terrane Mapping in the eastern Irumide and Mozambique belts: Implications for the assembly and dispersal of Rodinia, in McCourt, S., editor, IGCP $4184^{\text {th }}$ fieldmeeting: Durban, South Africa, University of Durban-Westville, p. 10-11.

Mapani, B., Rivers, T., Tembo, F., De Waele, B., and Katongo, C., 2004, Growth of the Irumide terranes and slices of Archaean age in eastern Zambia: Johannesburg, South Africa, Geoscience Africa 2004, p. $414-415$.

Mosley, P. N., and Marten, B. S., 1979, The Geology of the Katibunga mission area, explanation of degree sheet 1131, SE quarter: Lusaka, Geological Survey Department of Zambia.

Ngoyi, K., Liégeois, J.-P., Demaiffe, D., and Dumont, P., 1991, Age tardi-ubendien (Protérozoïque inférieur) des dômes granitiques de l'arc cuprifére zaïro-zambien: Comptes Rendu de l'Academie de Sciences, v. 313, p. 83-89.

Oliver, G. J. H., Johnson, S. P., Williams, I. S., and Herd, D. A., 1998, Relict 1.4 Ga oceanic crust in the Zambezi Valley, northern Zimbabwe: Evidence for Mesoproterozoic supercontinental fragmentation: Geology, v. 26, p. 571-573, doi:10.1130/0091-7613(1998)026<0571:RGOCIT>2.3.CO;2.

Pesonen, L. J., Elming, S.-A., Mertanen, S., Pisarevsky, S., D’Agrella-Filho, M. S., Meert, J. G., Schmidt, P. W., Abrahamsen, N., and Bylund, G., 2003, Palaeomagnetic configuration of continents during the Proterozoic: Tectonophysics, v. 375, p. 289-324, doi:10.1016/S0040-1951(03)00343-3.

Pidgeon, R. T., Furfaro, D., Kennedy, A. K., Nemchin, A. A., and Van Bronswijk, W., 1994, Calibration of zircon standards for the Curtin SHRIMP II: United States Geological Survey circular, v. 1107, p. 251.

Rainaud, C., Master, S., Armstrong, R. A., and Robb, L. J., 2003, A cryptic Mesoarchean terrane in the basement to the central African Copperbelt: London, Journal of the Geological Society, v. 160, p. 11-14, doi:10.1144/0016-764902-087.

2005, Geochronology and nature of the Palaeoproterozoic basement in the Central African Copperbelt (Zambia and the Democratic Republic of Congo), with regional implications: Journal of African Earth Sciences, v. 42, p. 1-31.

Rainaud, C. L., Armstrong, R. A., Master, S., Robb, L. J., and Mumba, P. A. C. C., 2002, Contributions to the geology and mineralisation of the central African Copperbelt: I. Nature and geochronology of the pre-Katangan basement: Windhoek, Namibia, Geological Survey of Namibia, 11th IAGOD Quadrennial Symposium and Geocongress, p. 5.

Rämö, O. T., McLemore, V. T., Hamilton, M. A., Kosunen, P. J., Heizler, M., and Haapala, I., 2003, Intermittent 1630-1220 Ma magmatism in central Mazatzal Province: new geochronologic piercing points and some tectonic implications: Geology, v. 31, p. 335-338, doi:10.1130/00917613(2003) 031<0335:IMMICM>2.0.CO;2.

Ring, U., Kröner, A., and Toulkeredis, T., 1997, Palaeoproterozoic granulite-facies metamorphism and granitoid intrusions in the Ubendian-Usagaran Orogen of northern Malawi, east-central Africa: Precambrian Research, v. 85, p. 27-51, doi:10.1016/S0301-9268(97)00028-4.

Ring, U., Kröner, A., Layer, P., Buchwaldt, R., and Toulkeredis, T., 1999, Deformed A-type granites in northern Malawi, east-central Africa: pre- or syntectonic: London, Journal of the Geological Society, v. 156 , p. $695-714$, doi:10.1144/gsigs.156.4.0695.

Rivers, T., 1997, Lithotectonic elements of the Grenville Province: review and tectonic implications: Precambrian Research, v. 86, p. 117-154, doi:10.1016/S0301-9268(97)00038-7.

Rivers, T., and Corrigan, D., 2000, Convergent margin on southeastern Laurentia during the Mesoproterozoic: tectonic implications: Canadian Journal of Earth Sciences, v. 37, p. 359-383, doi:10.1139/cjes-37-23-359.

Romer, R. L., 1996, Contiguous Laurentia and Baltica before the Grenvillian-Sveconorwegian orogeny?: Terra Nova, v. 8, p. 173-181, doi:10.1111/j.1365-3121.1996.tb00741.x

Schenk, V., and Appel, P., 2001, Anti-clockwise P-T path during ultrahigh-temperature (UHT) metamorphism at ca. $1050 \mathrm{Ma}$ in the Irumide Belt of Eastern Zambia: Berichte der Deutschen Mineralogischen Gesellschaft, Beihefte zum European Journal of Mineralogy, v. 13, p. 161.

Seth, B., Kröner, A., Mezger, K., Nemchin, A. A., Pidgeon, R. T., and Okrusch, M., 1998, Archaean to Neoproterozoic magmatic events in the Kaoko belt of NW Namibia and their geodynamic significance: Precambrian Research, v. 92, p. 341-363, doi:10.1016/S0301-9268(98)00086-2.

Seth, B., Armstrong, R. A., Brandt, S., Villa, I. M., and Kramers, J. D., 2003, Mesoproterozoic U-Pb and $\mathrm{Pb}-\mathrm{Pb}$ ages of granulites in NW Namibia: reconstructing a complete orogenic cycle: Precambrian Research, v. 126, p. 147-168, doi:10.1016/S0301-9268(03)00193-1.

Seth, B., Armstrong, R. A., Büttner, A., and Villab, I. M., 2005, Time constraints for Mesoproterozoic upper amphibolite facies metamorphism in NW Namibia: a multi-isotopic approach: Earth and Planetary Science Letters, v. 230, p. 355-378, doi:10.1016/j.eps1.2004.11.022. 
Smith, A. G., 1966, The geology of the Kapiri Mposhi area; explanation of degree sheet 1328, SE quarter: Lusaka, Geological Survey Department of Zambia, p. 32.

Stacey, J. S., and Kramers, J. D., 1975, Approximation of terrestrial lead isotopic evolution by a two-stage model: Earth and Planetary Science Letters, v. 26, p. 207-221, doi:10.1016/0012-821X(75)90088-6.

Stillman, C. J., 1965, The geology of the Musofu River and Mkushi areas; explanation of degree sheet 1329 part of NW quarter and SW quarter: Lusaka, Geological Survey Department of Zambia, p. 52.

Sykes, J. M. D., 1995, The geology of the Luswa River area, degree sheet 1132, NW quarter: Lusaka, Geological Survey Department, p. 20.

Tack, L., Fernandez-Alonso, M., Tahon, M., Wingate, M. T. D., and Barritt, S., 2002, The "northeastern Kibaran belt" (NKB) and its mineralisations reconsidered: new constraints from a revised lithostratigraphy, a GIS-compilation of existing geological maps and a review of recently published as well as unpublished igneous emplacement ages in Burundi, in Geological Survey of Namibia, editor, 11th IAGOD Quadrennial Symposium and Geocongress: Windhoek, Namibia, Geological Survey of Namibia, p. 6.

Unrug, R., 1992, Kibaran terranes with cassiterite mineralisation in the Zambezi belt, Zambia and Zimbabwe: IGCP Bulletin, v. 4, p. 121-124.

Van Tuijl, M. M., and Verhoog, B. J., 1995, The geology of the Kalungu and Mututa Hills area; explanation of degree sheet 0932, SE and SW quarter: Lusaka, Geological Survey Department of Zambia.

Verhoog, B. J., and Van Tuijl, M. M., 1995a, The geology of the Chozi area; explanation of degree sheet 0932, NW quarter: Lusaka, Geological Survey Department of Zambia.

$1995 \mathrm{~b}$, The geology of the Nakonde area; explanation of degree sheet $0932 \mathrm{NE}$ quarter: Lusaka, Geological Survey Department of Zambia.

Vrána, S., Kachlík, V., Kröner, A., Marheine, D., Seifert, A. V., Zácek, V., and Baburek, J., 2004, Ubendian basement and its late Mesoproterozoic and early Neoproterozoic structural and metamorphic overprint in northeastern Zambia: Journal of African Earth Sciences, v. 38, p. 1-21, doi:10.1016/j. jafrearsci.2003.09.001.

Westerhof, A. B. P., Lehtonen, M. I., Mäkitie, H., Manninen, T., Pekkala, Y., Gustafsson, B., and Tahon, A. 2008, The Tete-Chipata Belt: A New Multiple Terrane Element from Western Mozambique and Southern Zambia, in Pekkala, Y., Lehto, T., and Mäkitie, H., editors, GTK Consortium Geological Surveys in Mozambique 2002-2007: Geological Survey of Finland, Special Paper 48, p. 145-166. 